\title{
Synthesis of Epoxides from Alkyl Bromides and Alcohols with In Situ Generation of Dimethyl Sulfonium Ylide in DMSO Oxidations
}

Zhi-Wei Zhang, * Hai-Bo Li, Jin Li, Cui-Cui Wang, Juan Feng, Yi-Hua Yang and Shouxin Liu*

State Key Laboratory Breeding Base-Hebei Province Key Laboratory of Molecular Chemistry for

Drug, College of Chemical \& Pharmaceutical Engineering, Hebei University of Science \&

Technology, Shijiazhuang 050018, P. R. China

E-mail: zhangzw@hebust.edu.cn

E-mail: chlsx@hebust.edu.cn

\section{Table of Content}

${ }^{1} \mathrm{H}$ and ${ }^{13} \mathrm{C}$ NMR spectra..

S2 


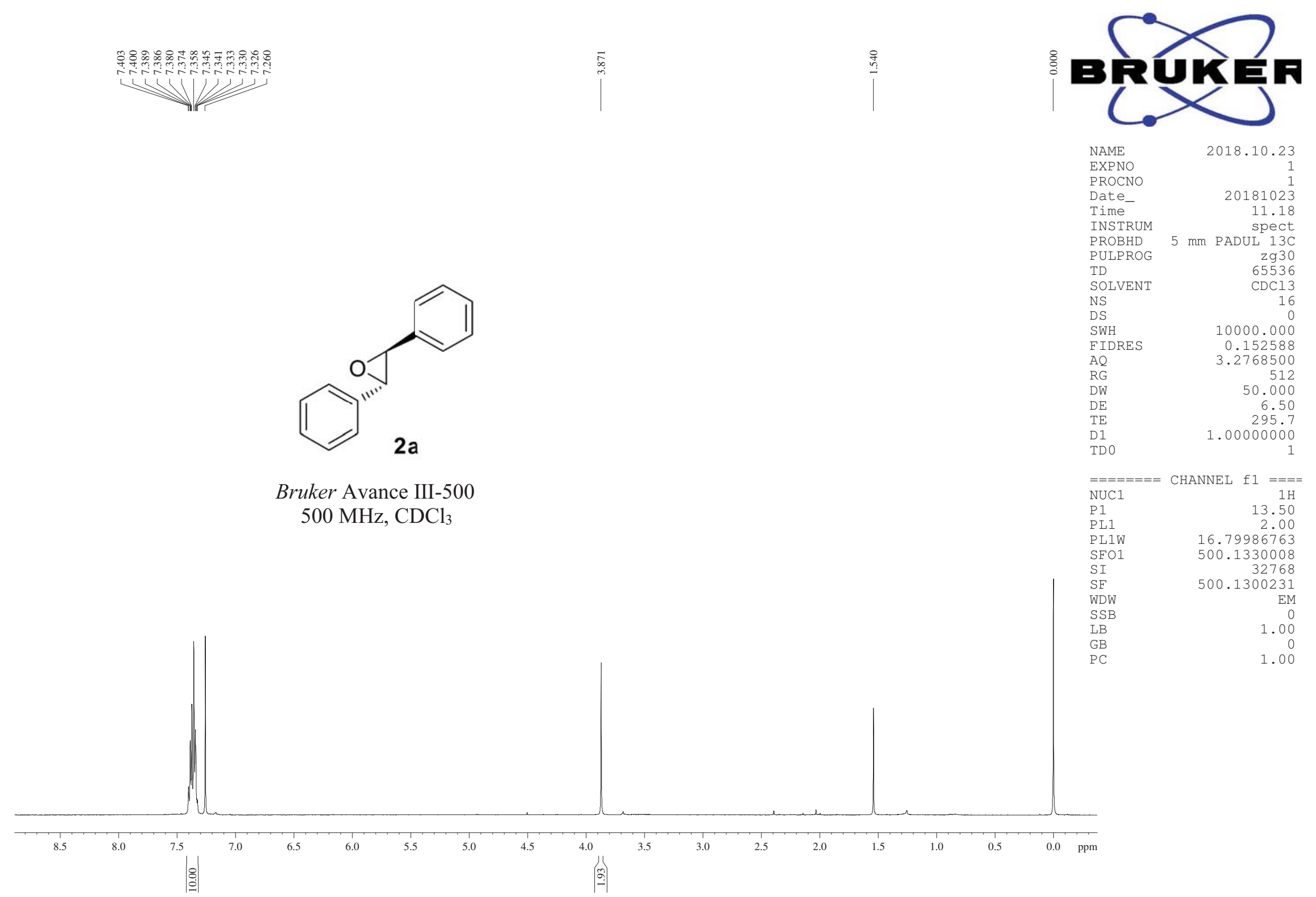




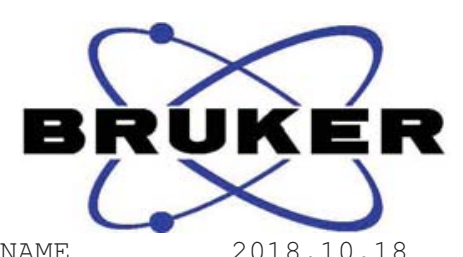

AAME

ROCNO

2018.10 .18

Date

20181018

22.06

spect
S

PULPROG zgpg30

TD 32768

CDCl3
SOLVENT

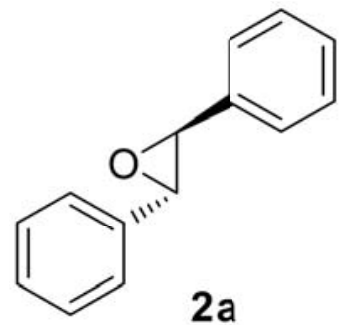

DS

SWH $\quad 29761.904 \mathrm{~Hz}$

FIDRES $\quad 0.908261 \mathrm{~Hz}$

$\begin{array}{lr}\mathrm{AQ} & 0.5505524 \mathrm{sec} \\ \mathrm{RG} & 1150\end{array}$

$\mathrm{DW}$

$\mathrm{DE}$

TE

D11

16.800 usec

6.50 usec
$297.0 \mathrm{~K}$

$.00000000 \mathrm{sec}$

$0.03000000 \mathrm{sec}$

Bruker Avance III-500

$=======$ CHANNEL 1

NUC1

$125 \mathrm{MHz}, \mathrm{CDCl}_{3}$

P1

PL1

PL1W
SFO1

$=======$

$13 \mathrm{C}$

$4.50 \mathrm{~dB}$

$\begin{array}{ll} & 33.60015869 \mathrm{~W} \\ \text { SFO1 } & 125.7703643 \mathrm{MHz}\end{array}$

CPDPRG2 waltzl6

NUC2

PL2

PL12

PL13

PL2W

PL12W

PL13W

$\mathrm{SFO} 2$

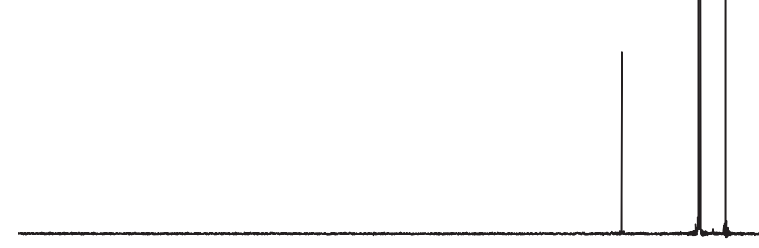

SE
WDW

WDW
SSB

SSB
LB 


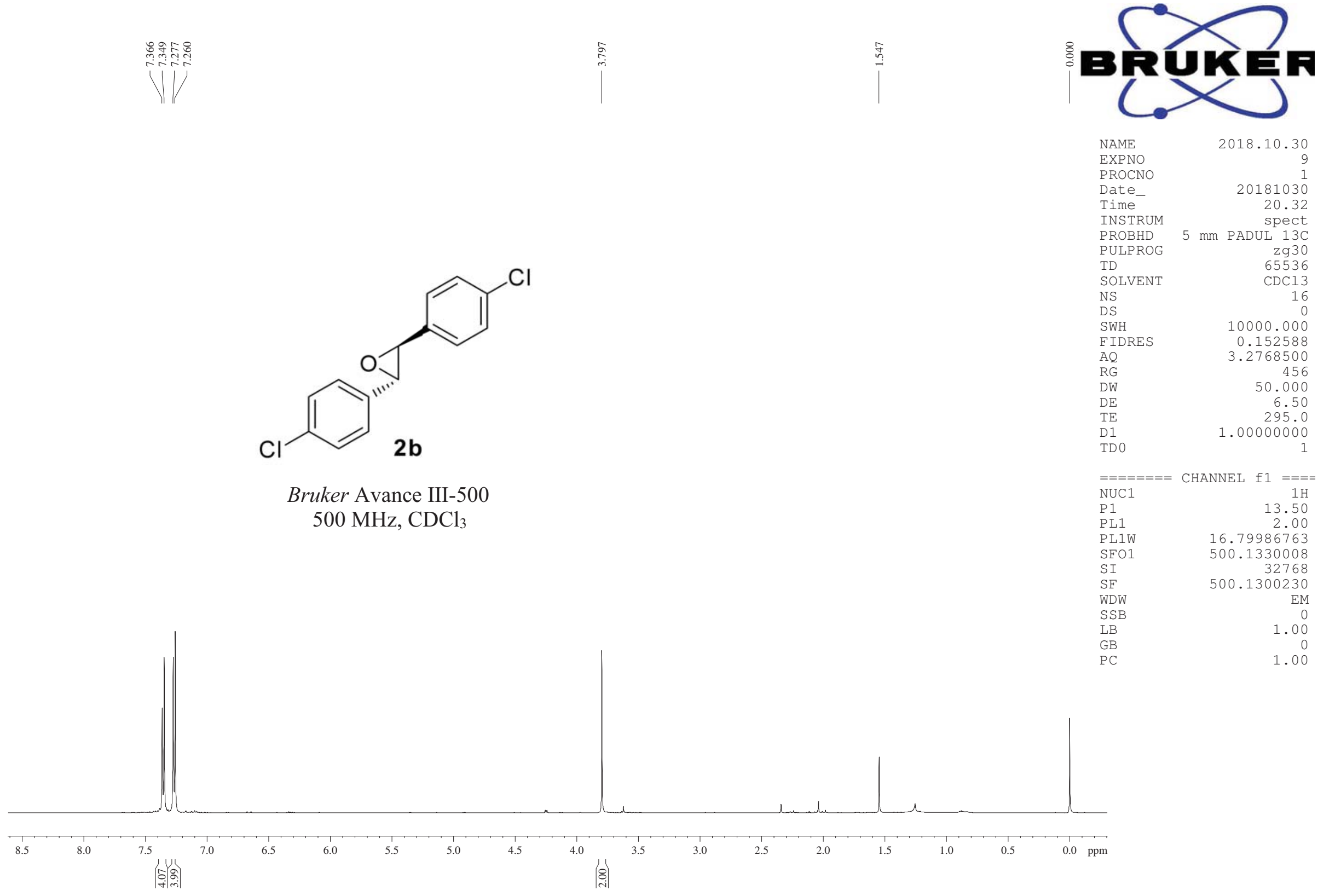




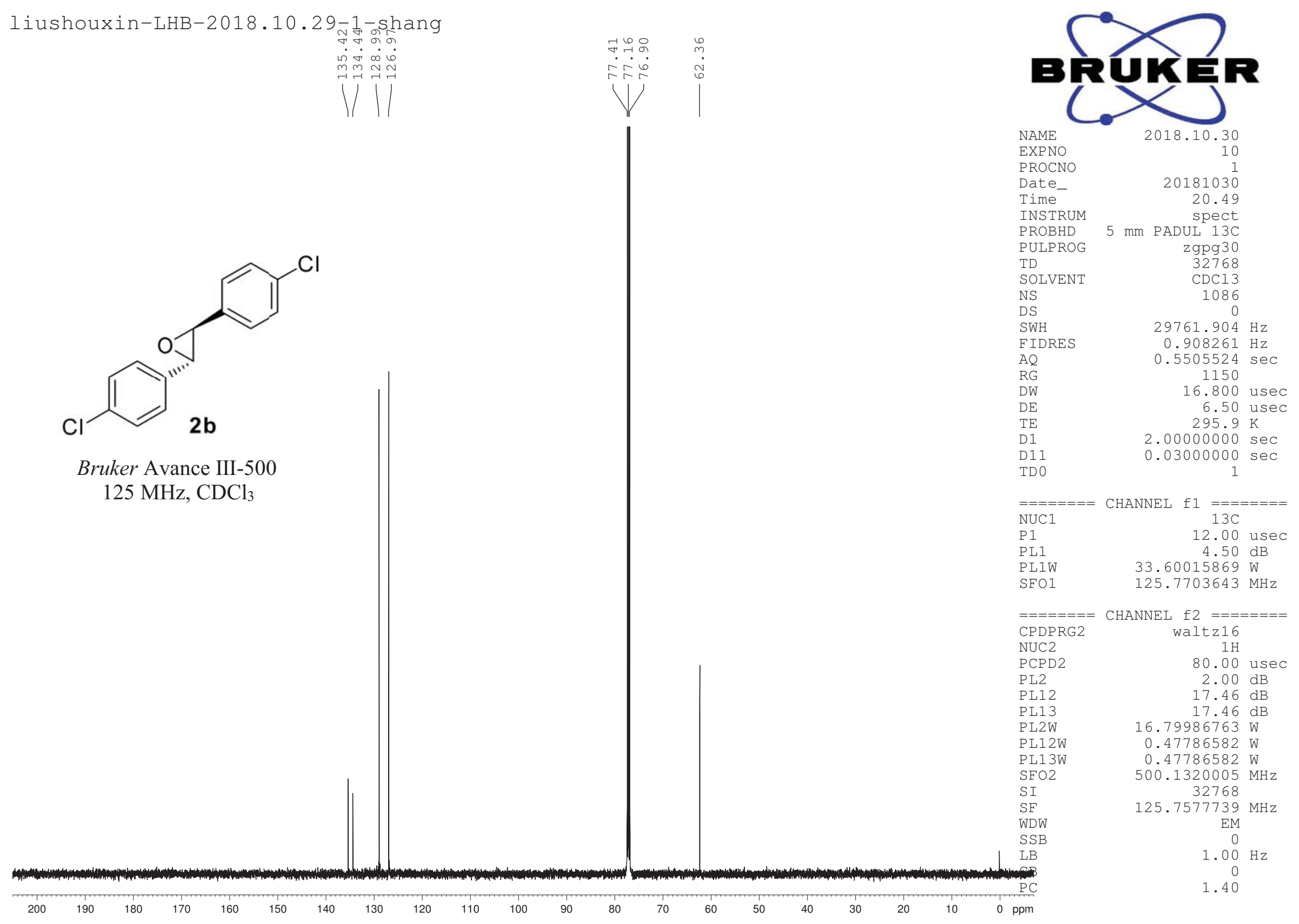




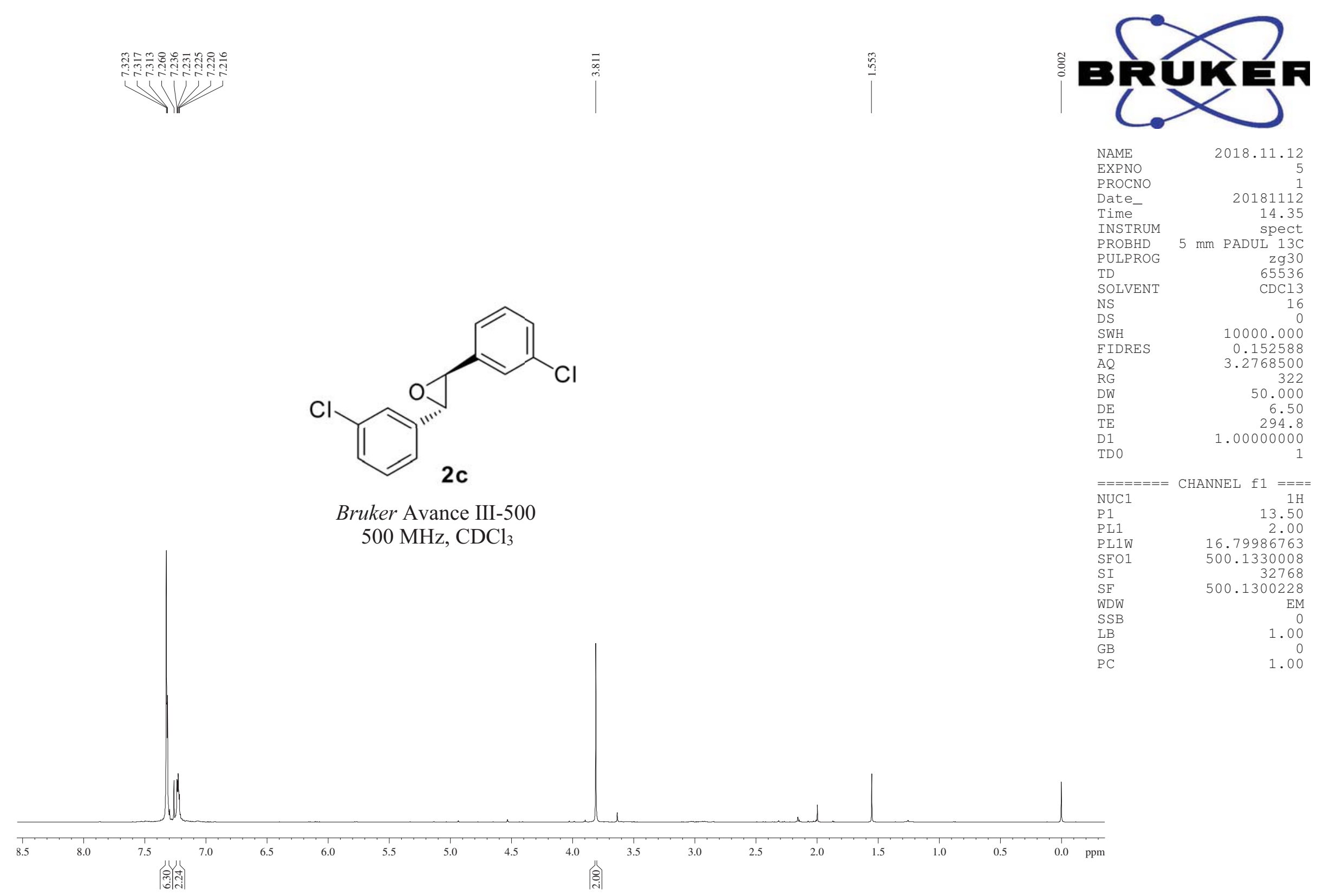




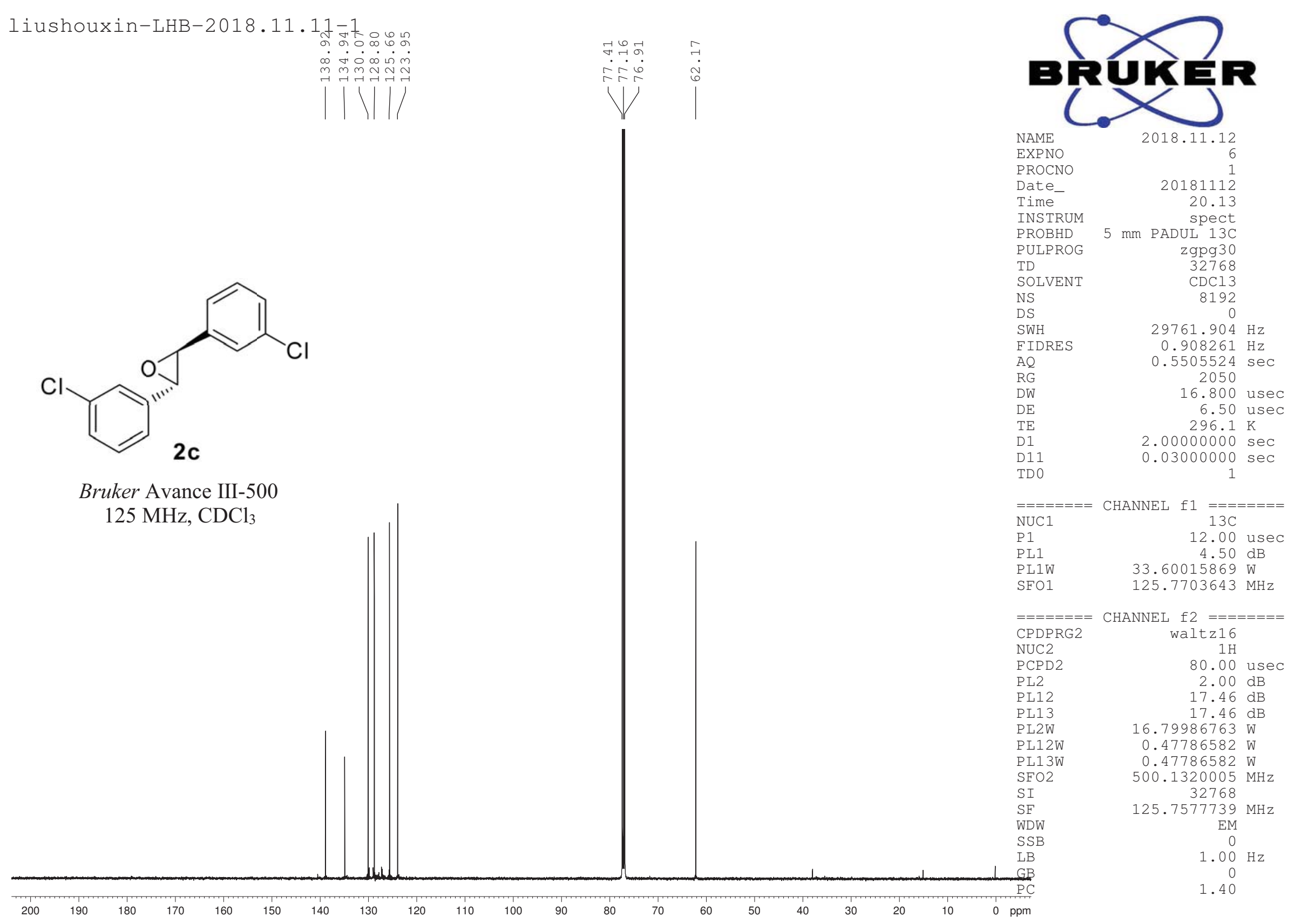




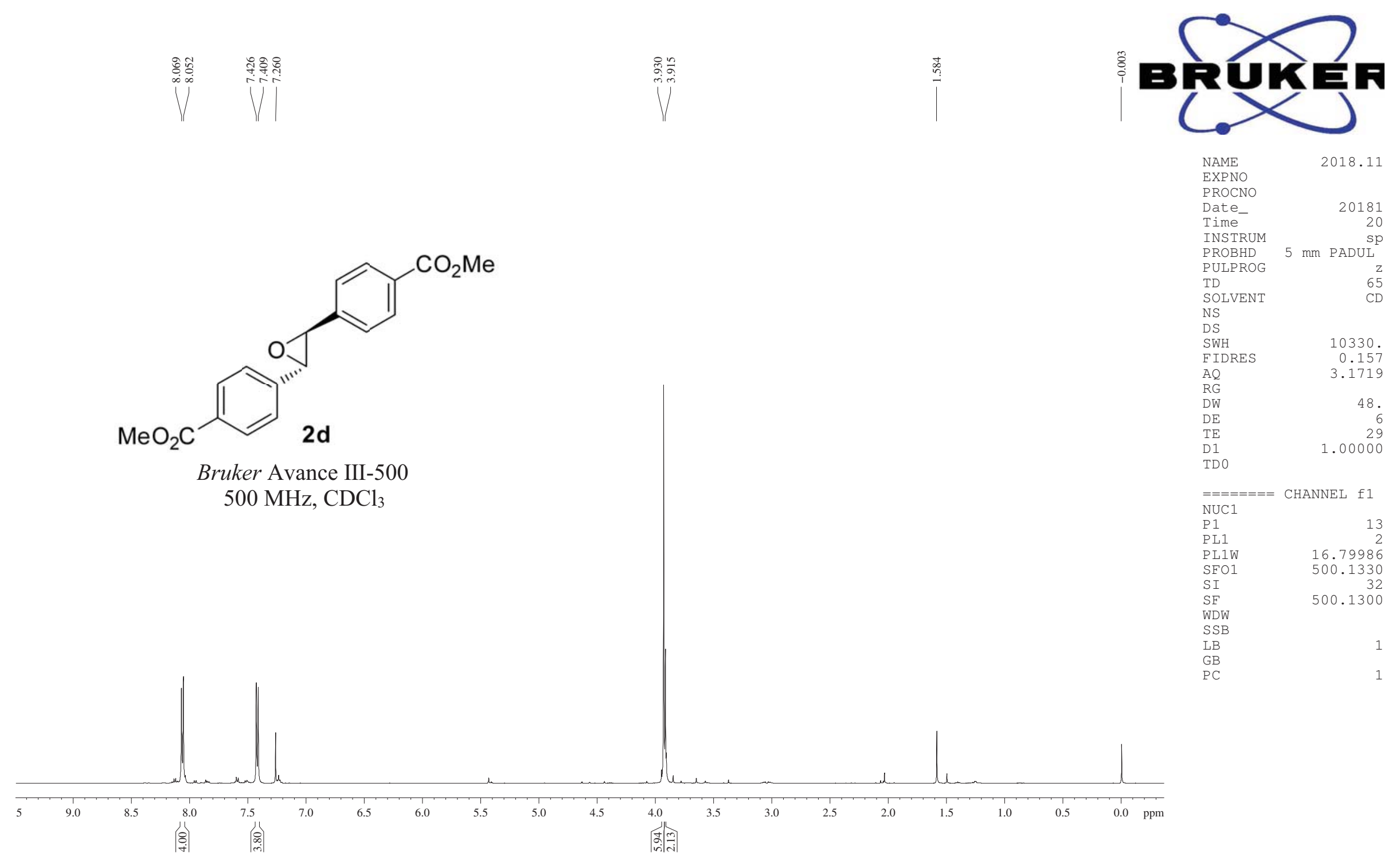




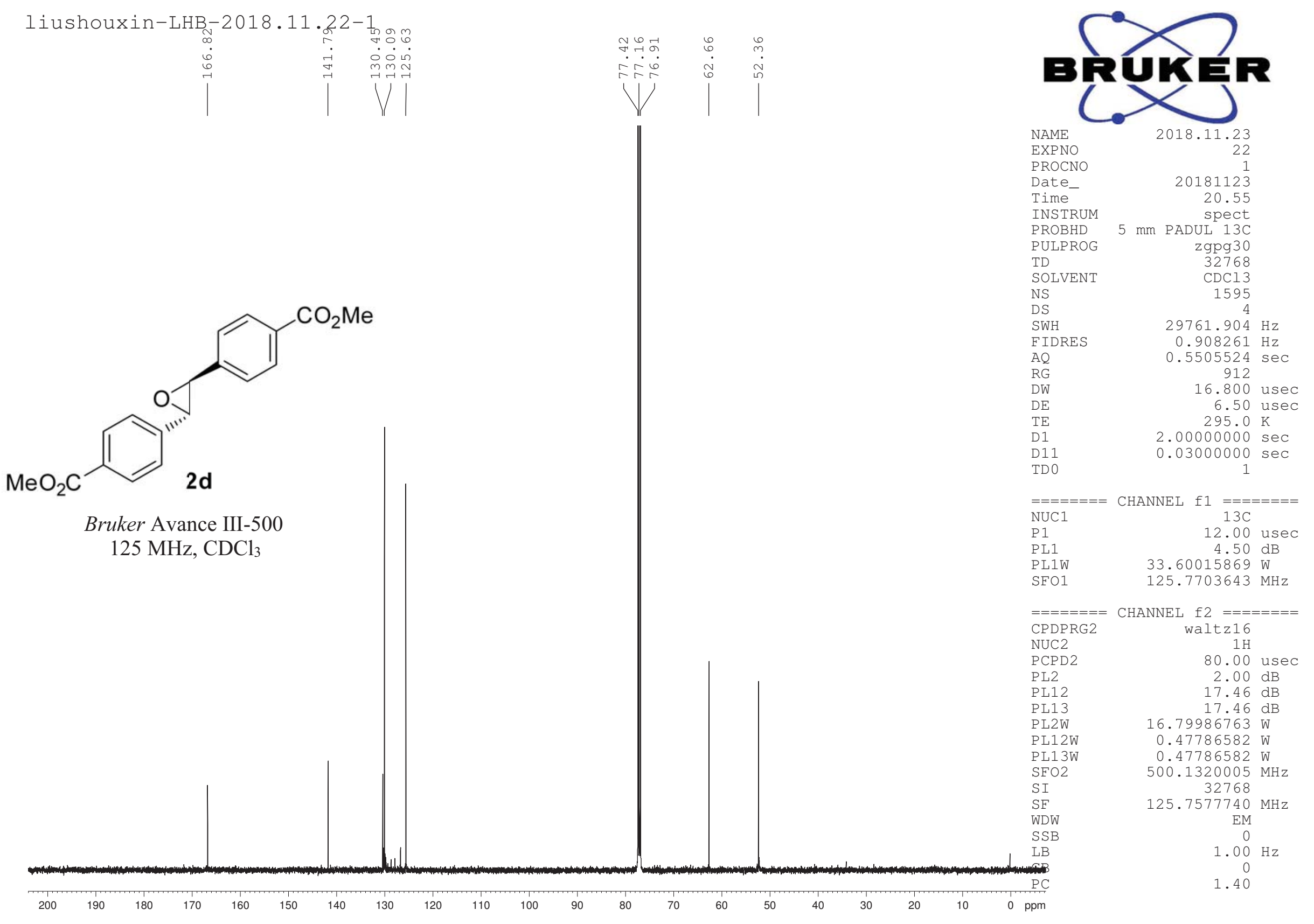




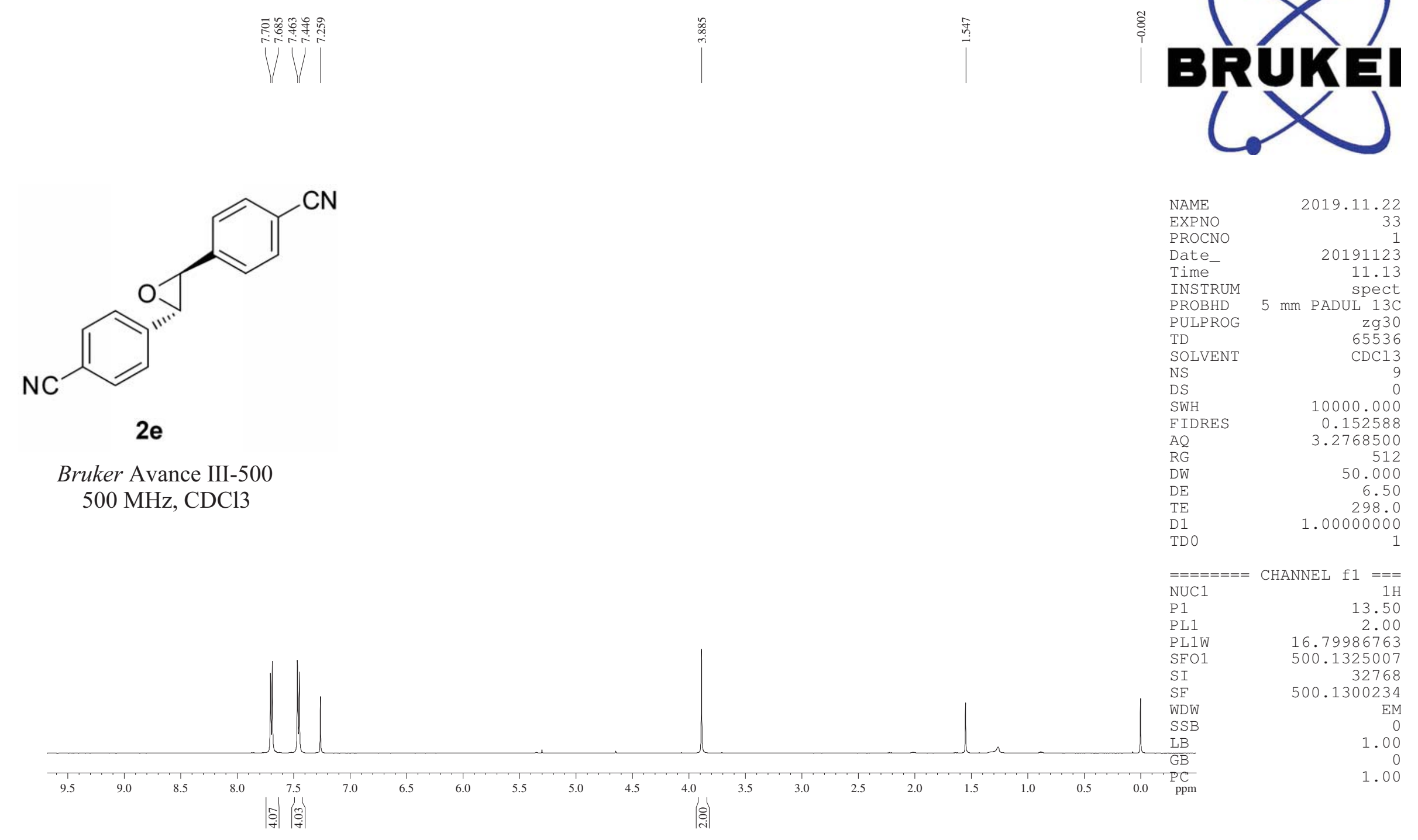




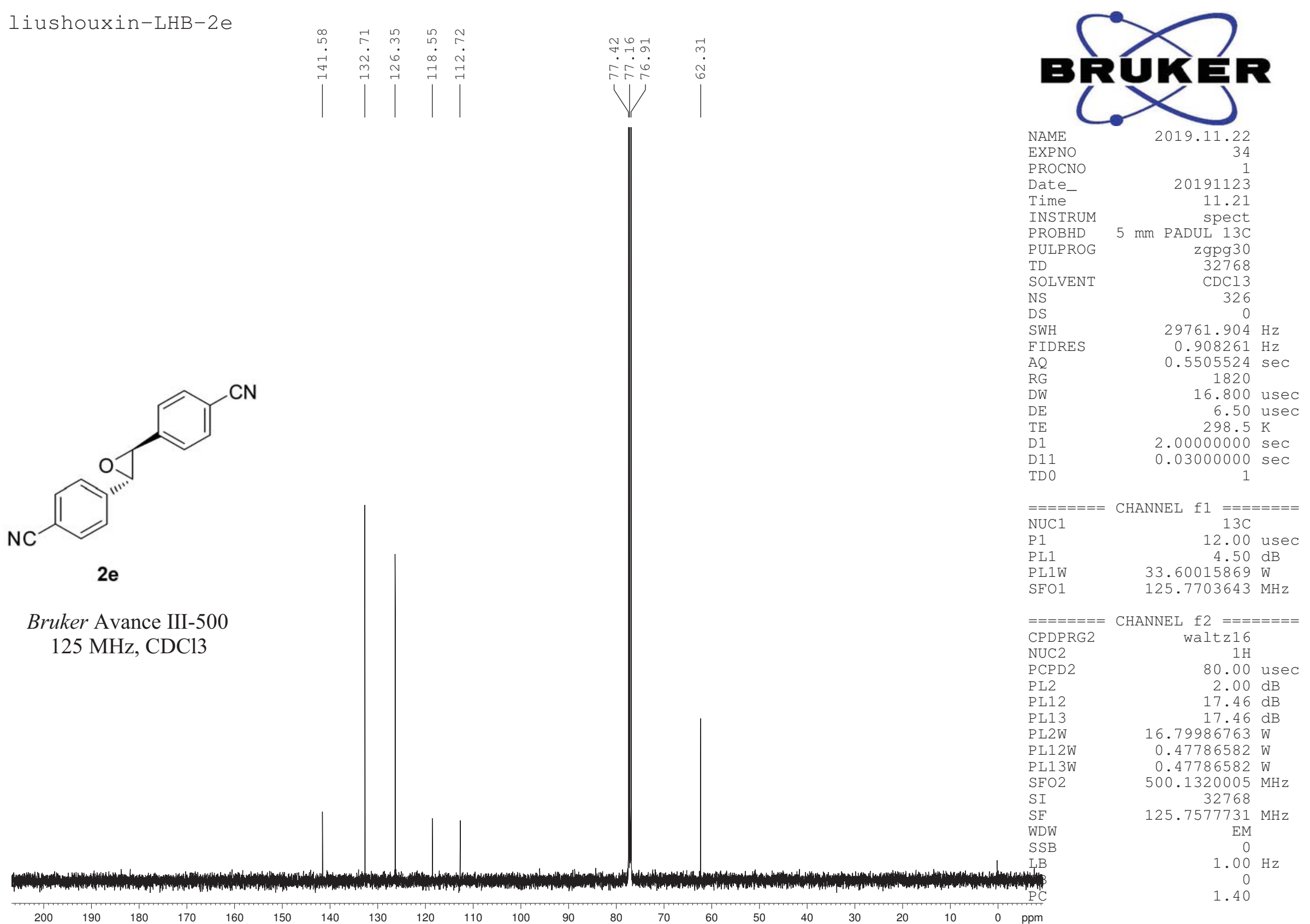




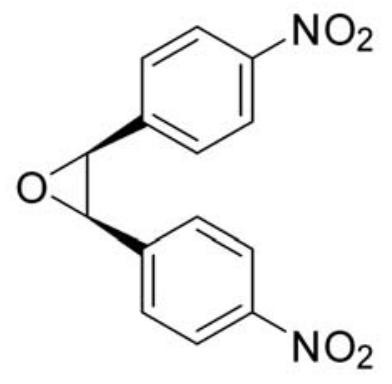

$2 f$

Bruker Avance III-500 $500 \mathrm{MHz}, \mathrm{CDCl} 3$

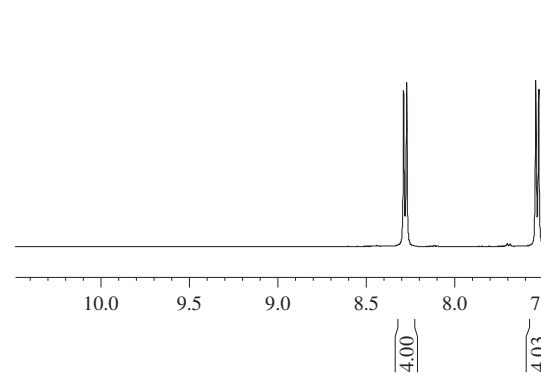

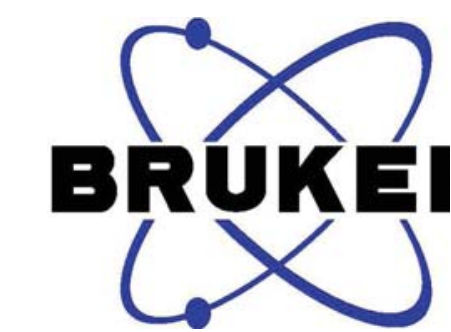

NAME

EXPNO

PROCNO

Date_-

INSTRUM

PROBHD

PULPROG

TD
SOLVENT

NS
DS

SWH

FIDRES

AQ
RG
DW

DW
DE

TE

D1
TD 0

2019.11 .23

1

20191124
20.59

spect

mm PADUL $13 \mathrm{C}$

$\mathrm{zg} 30$
65536

$\mathrm{CDCl} 3$

10000.000

0.152588

3.2768500

50.000

6.50

1.00000000

$=====-=$ CHanof fi

NUC1

P1

PL1

PL1W
SFO1

SFO

SI

WDW

WDW
SSB

${ }_{\mathrm{SB}}^{\mathrm{SSB}}$

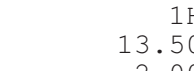
00.1325007 32768
500.1300228

$\mathrm{EM}$
0
1.00

1.00 


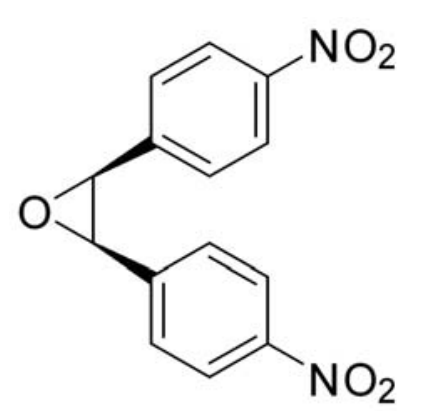

$2 f$

Bruker Avance III-500 $125 \mathrm{MHz}, \mathrm{CDCl} 3$

\section{Som}

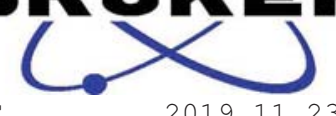

NAME

PROCNO

Time

20191124

INSTRUM 21.10

PROBHD $5 \mathrm{~mm}$ PADUL 13C

$\begin{array}{lr}\text { PULPROG } & \text { zgpg30 } \\ \text { TD } & 32768\end{array}$

SOLVENT

NS

DS

SWH 29761.904 Hz

FIDRES $\quad 0.908261 \mathrm{~Hz}$

$\begin{array}{lr}\text { AQ } & 0.5505524 \mathrm{sec} \\ \text { RG } & 1150\end{array}$

$\mathrm{DW}$

DE

TE
D1
D11

TD 0

16.800 usec

6.50 usec

$0.03000000 \mathrm{sec}$

$======$ CHANNEL

NUC1

PL1

PL1W
SFO1

$========$

$13 \mathrm{C}$

$4.50 \mathrm{~dB}$

$33.60015869 \mathrm{~W}$

$125.7703643 \mathrm{MHz}$

$=======$ CHANNEL $\mathrm{f} 2$ ========

CPDPRG2 waltz16

NUC2

PL2

PL12

$\mathrm{PL} 13$

PL2W

PL12W

PL13W

$\mathrm{SFO} 2$

SI

SF
WDW

S.SB

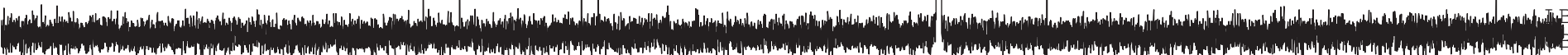

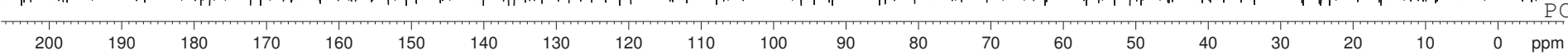

80.00 usec

$2.00 \mathrm{~dB}$

$17.46 \mathrm{~dB}$

$17.46 \mathrm{~dB}$

$16.79986763 \mathrm{~W}$

$0.47786582 \mathrm{~W}$

$0.47786582 \mathrm{~W}$

$500.1320005 \mathrm{MHz}$

EM

0
1.0 $\mathrm{~Hz}$

0
1.40
$125.7577730 \mathrm{MHz}$ 


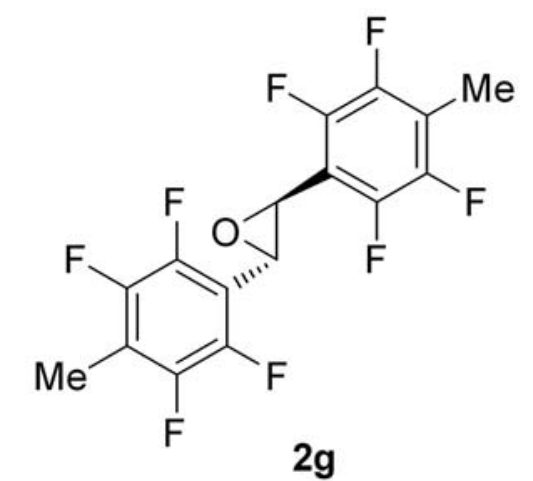

NAME

EXPNO

PROCNO

Date_-
Time

INSTRUM

PROBHD PULPROG

TD

$$
\begin{aligned}
& \text { SOL } \\
& \text { NS } \\
& \text { DS } \\
& \text { SWH }
\end{aligned}
$$

SWH
FIDRES

$\mathrm{AQ}$

RG

RG
DW
DE

Bruker Avance III-500 $500 \mathrm{MHz}, \mathrm{CDCl} 3$

D1

2019.11 .26

20191127 18.21 spect

$5 \mathrm{~mm}$ PADUL $13 \mathrm{C}$ zg30
65536 $\mathrm{CDCl} 3$

10000.000 0.152588 3.2768500

512
50.000

6.50

1.00000000

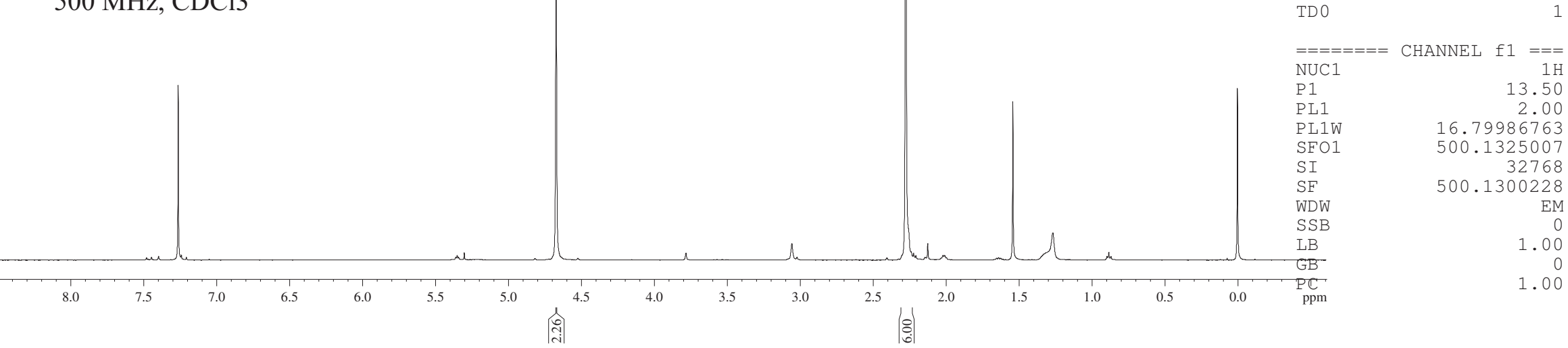




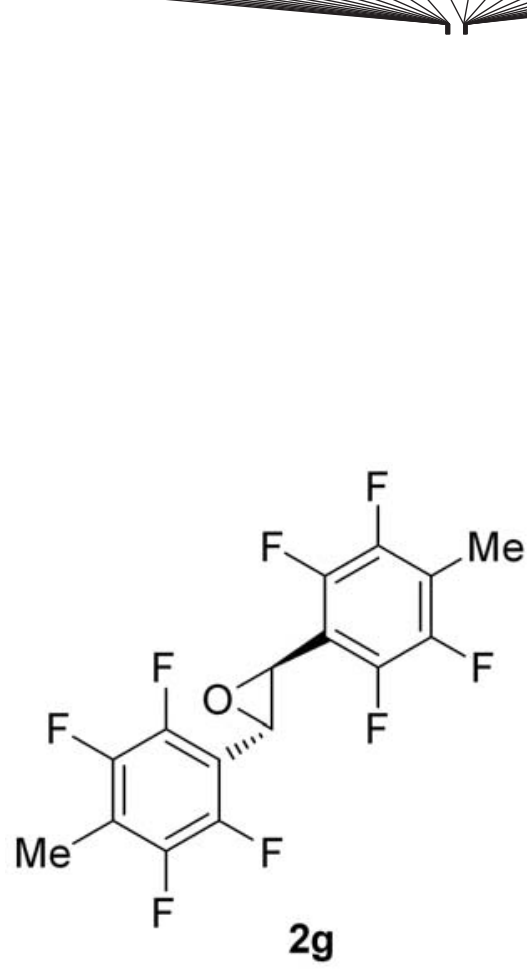

Bruker Avance III-500 $125 \mathrm{MHz}, \mathrm{CDCl} 3$
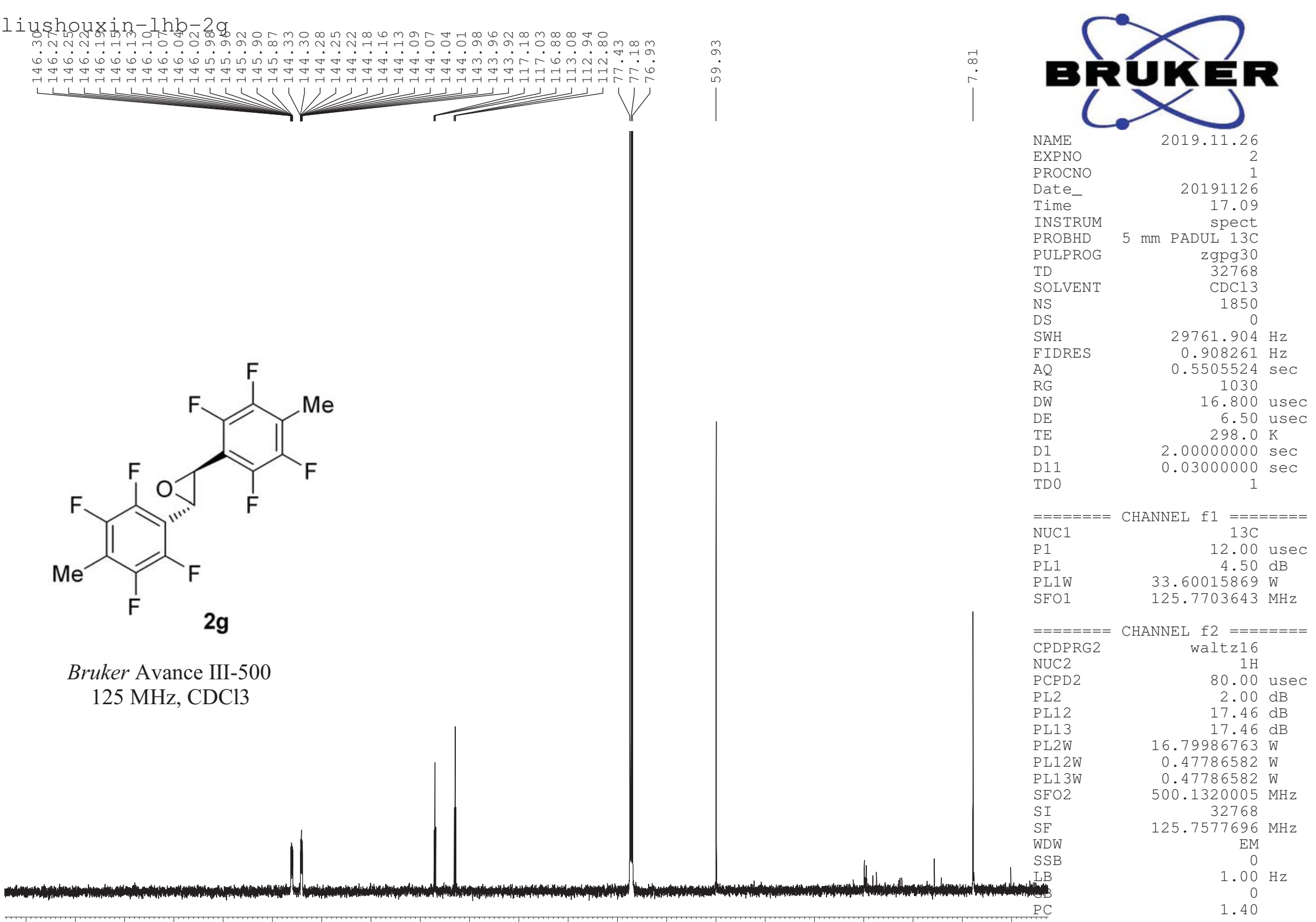


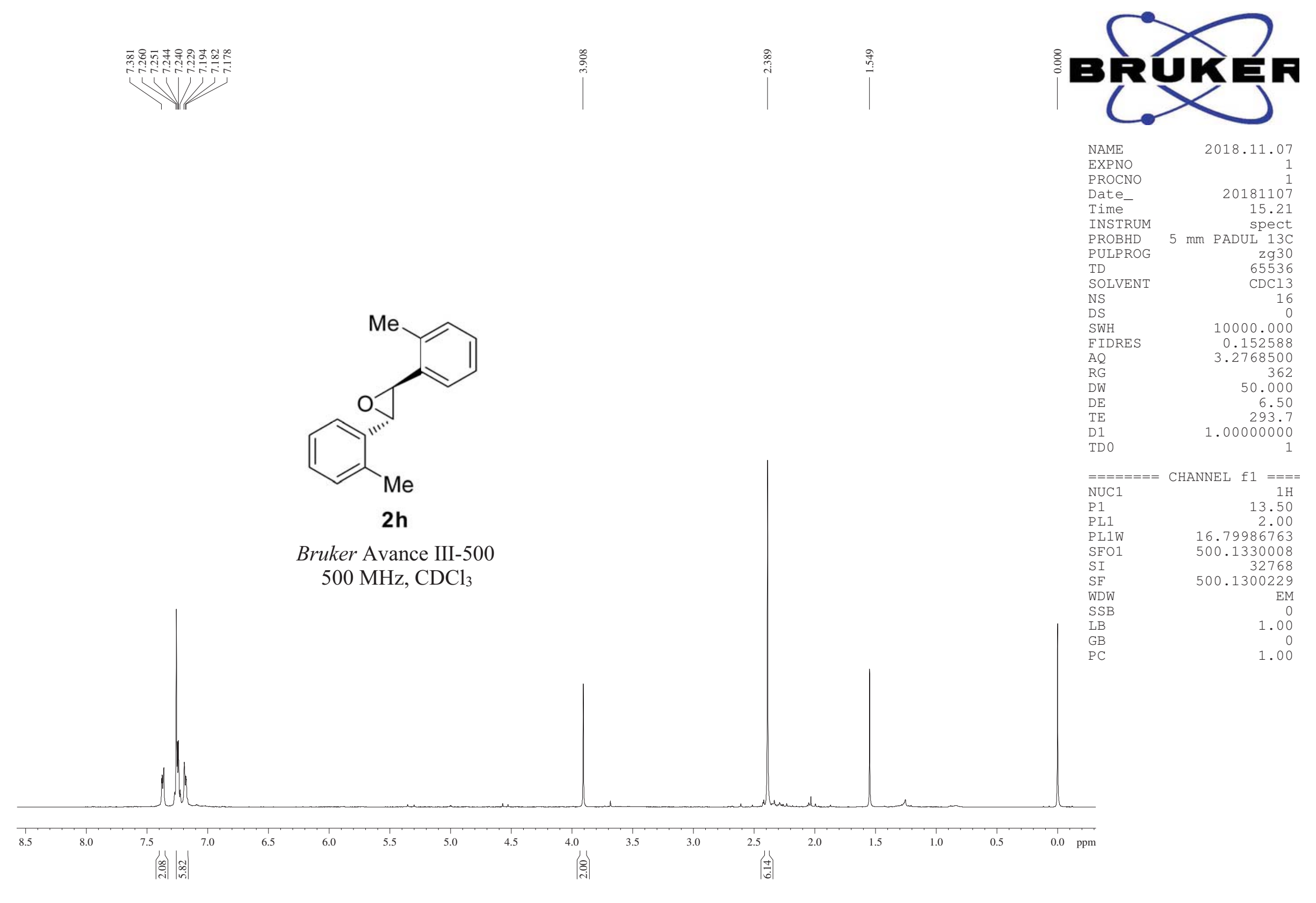




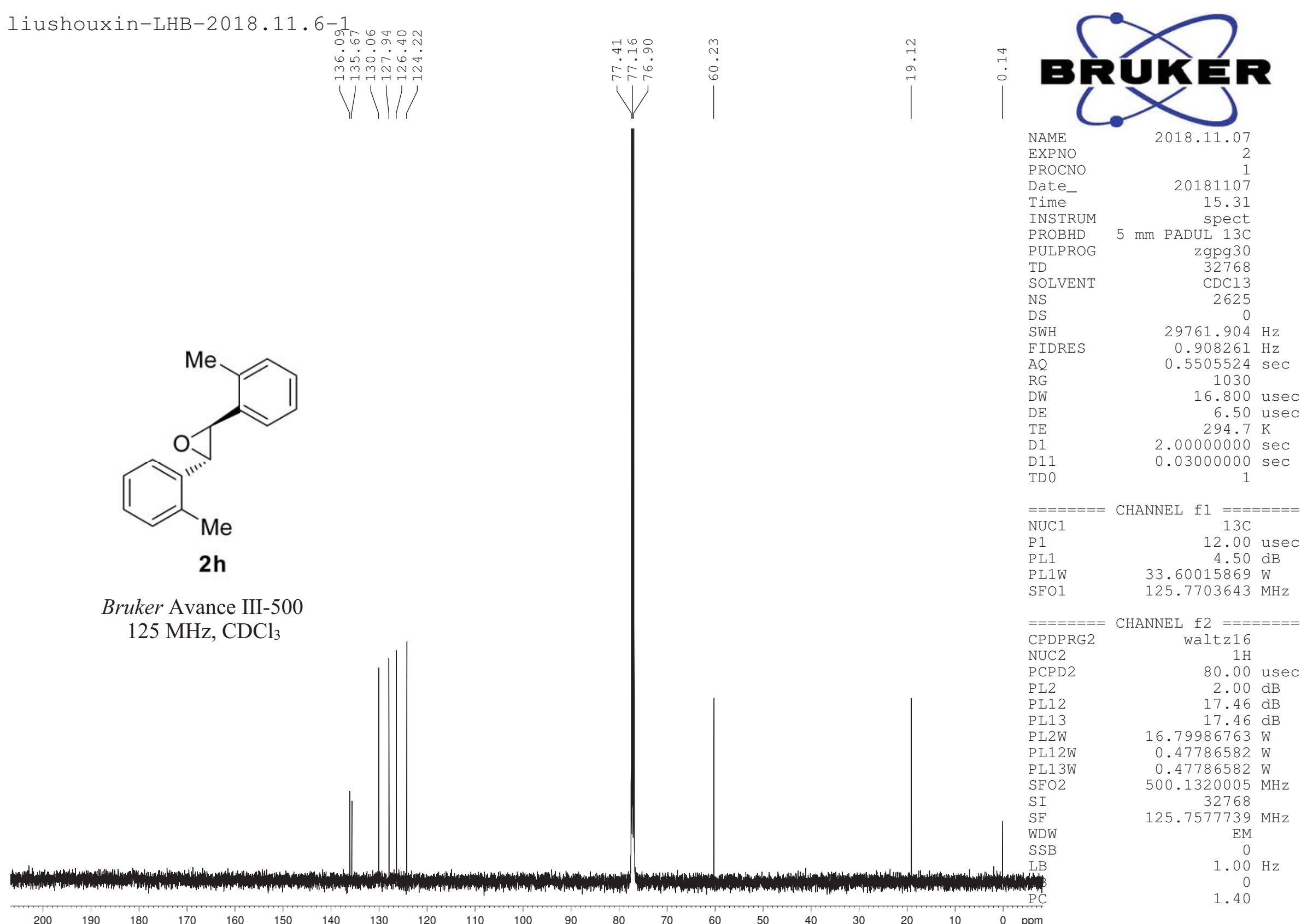




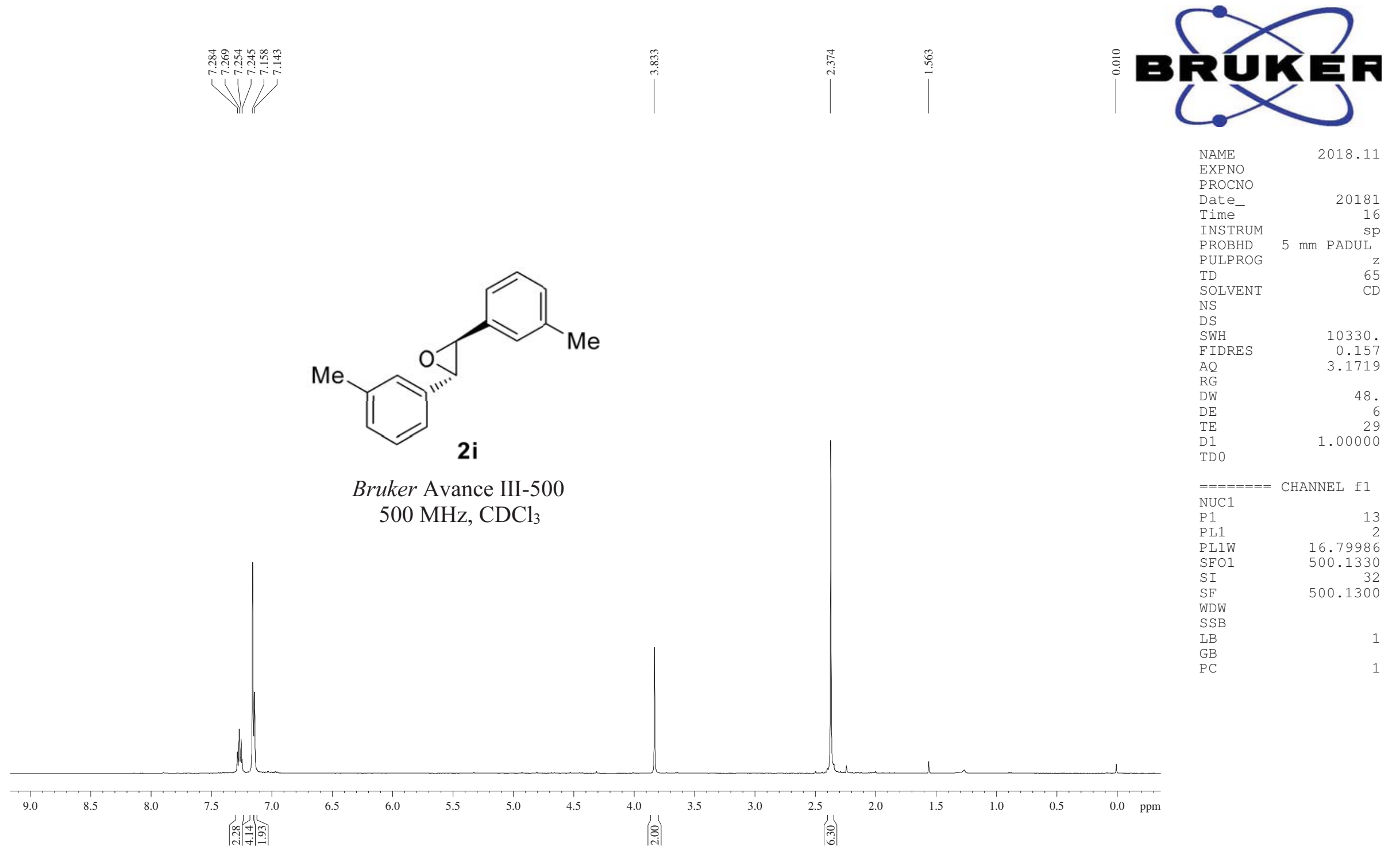




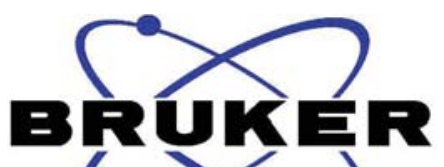
$(><)$

NAME

PROCNO

Date

Time-

INSTRUM

PROBHD

PULPROG

TD

SOLVENT

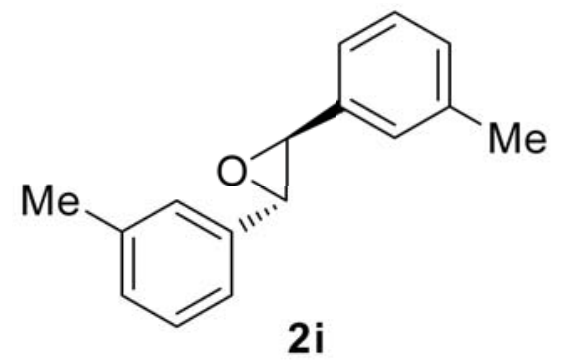

Me

Bruker Avance III-500 $125 \mathrm{MHz}, \mathrm{CDCl}_{3}$

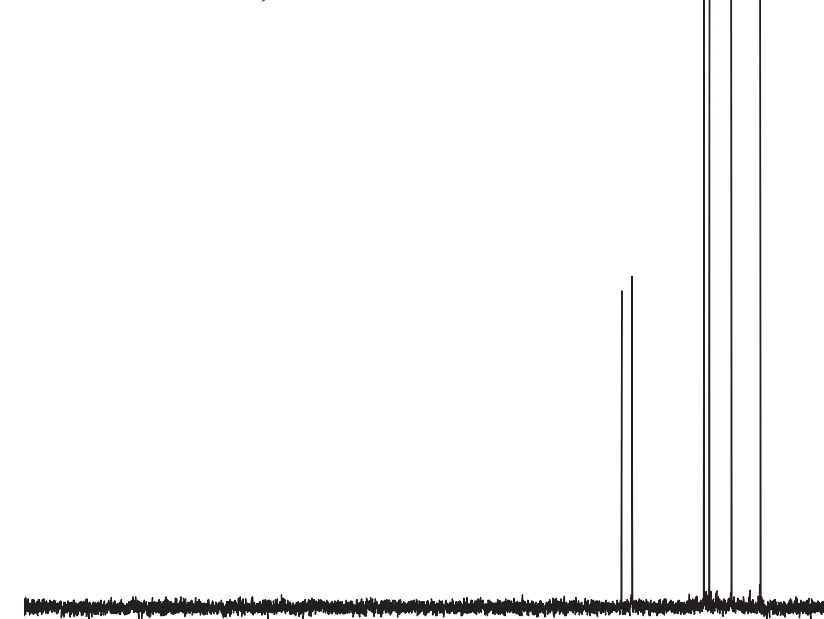

DS

SWH

$29761.904 \mathrm{~Hz}$

$\mathrm{AQ}$
$\mathrm{RG}$

DW

DE

D1

D11
TD 0

$=======$ CHANNEL

NUC1

P1

PL1
PL1W

$\mathrm{SFO1}$

20181121

16.20

$5 \mathrm{~mm}$ PADUL $13 \mathrm{C}$

zgpg 30
32768

$\mathrm{CDCl} 3$

$0.908261 \mathrm{~Hz}$

0.5505524 sec

16.800 usec

6.50 usec
$295.4 \mathrm{~K}$

$.00000000 \mathrm{sec}$

$0.03000000 \mathrm{sec}$

\section{$=======$}

CPDPRG

$\mathrm{NUC2}$

PCPD2

PL2
PL12

PL12
PL13

PL13
PL $2 W$

PL2W
PL12W

PL13W

SFO2

SI

$\mathrm{SF}$

WDW

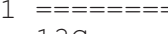
$13 \mathrm{C}$ 2.00 use
$4.50 \mathrm{~dB}$ $33.60015869 \mathrm{~W}$ $125.7703643 \mathrm{MHz}$ CHANNEL f 2 ======= waltz16 80.00 use $2.00 \mathrm{~dB}$ $17.46 \mathrm{~dB}$ $17.46 \mathrm{~dB}$ $16.79986763 \mathrm{~W}$ $0.47786582 \mathrm{~W}$ $0.47786582 \mathrm{~W}$ $500.1320005 \mathrm{MHz}$ $125.7577785 \mathrm{MH}$ $\mathrm{EM}$
0
$1.00 \mathrm{~Hz}$
0 1.40 


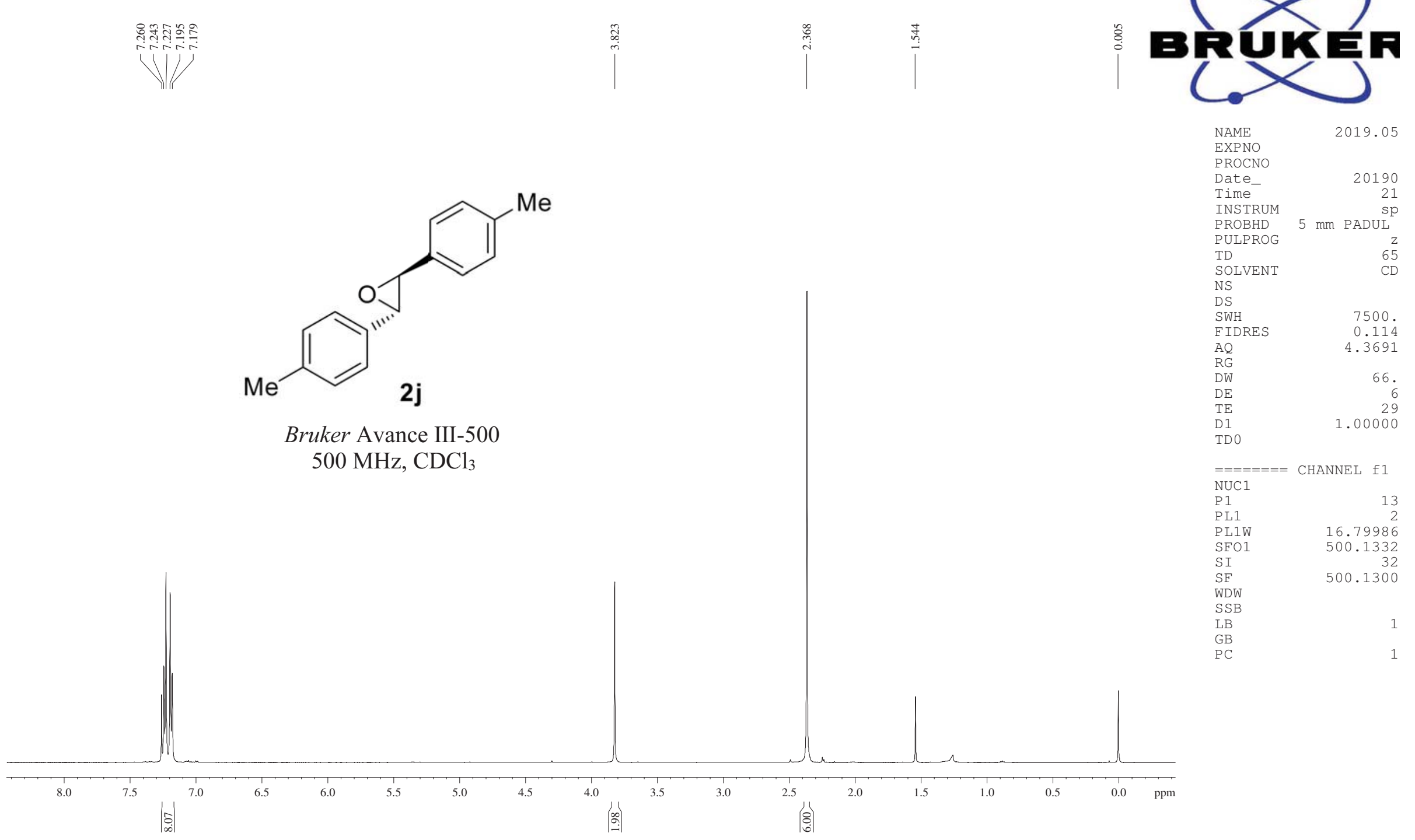




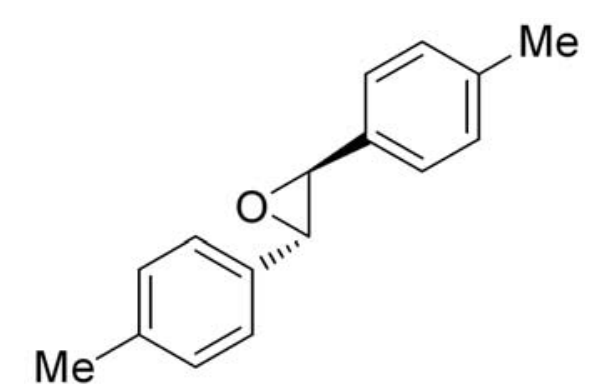

2j

Bruker Avance III-500 $125 \mathrm{MHz}, \mathrm{CDCl} 3$

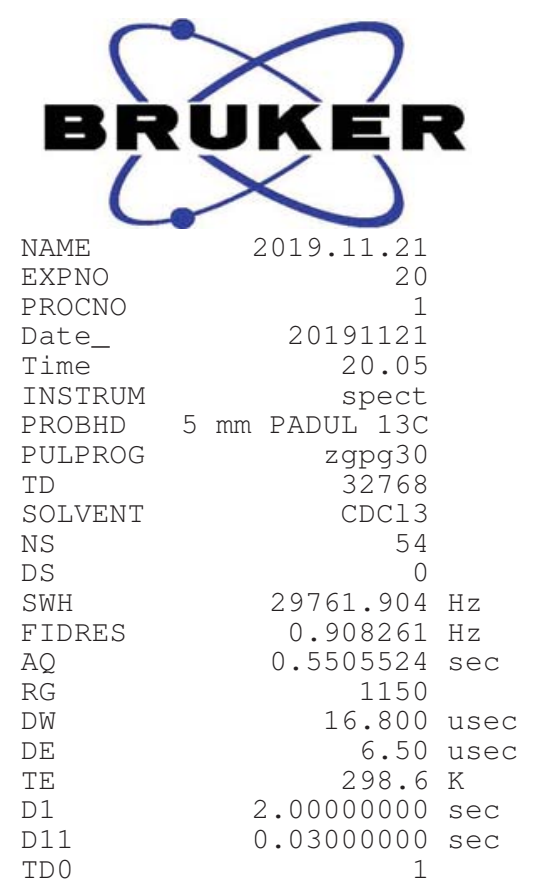

NUC1

P 1

PL1
PL1W

SFO1

$=======$

12.00 usec

$4.50 \mathrm{~dB}$

$33.60015869 \mathrm{~W}$

$125.7703643 \mathrm{MHz}$

CHANNEL $12====$

CPDPRG2 waltz16

$\mathrm{NUC2}$

PL2

PL12

$\mathrm{PL} 13$

PL2W

PL12W

PL13W

$\mathrm{SFO} 2$

SI

SF

WDW
SSB
LB

$S S B$
LB
80.00 use

$2.00 \mathrm{~dB}$

$17.46 \mathrm{~dB}$

$17.46 \mathrm{~dB}$

$16.79986763 \mathrm{~W}$

$0.47786582 \mathrm{~W}$

$50.47786582 \mathrm{~W}$

$500.1320005 \mathrm{MHz}$

$125.7577757 \mathrm{MH}$

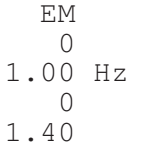

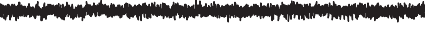
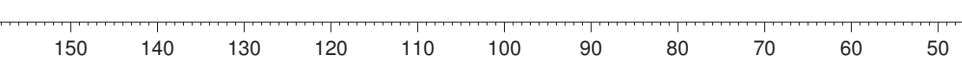


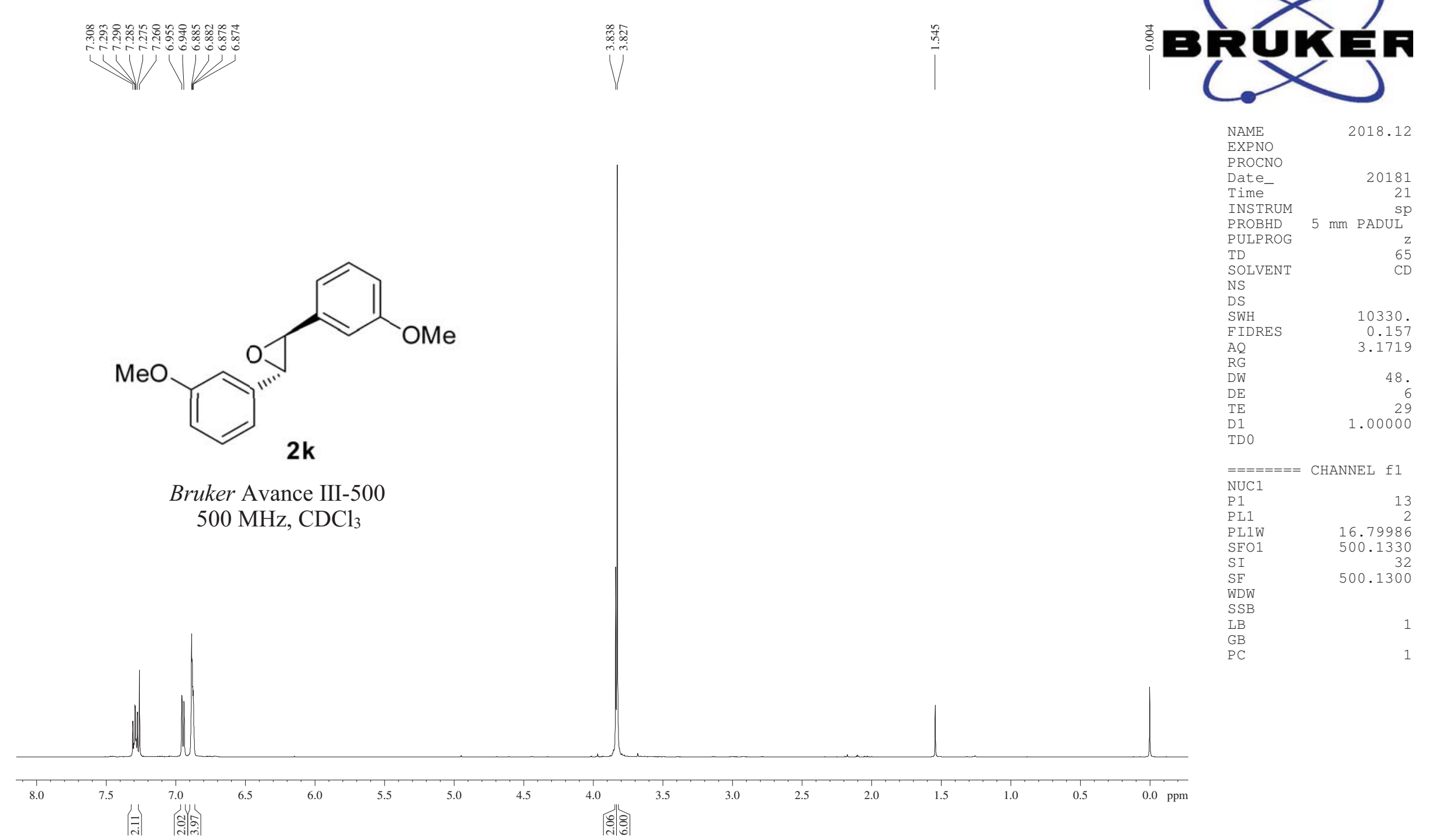




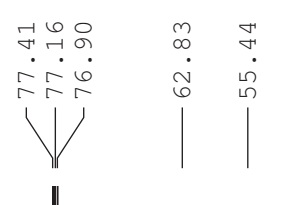

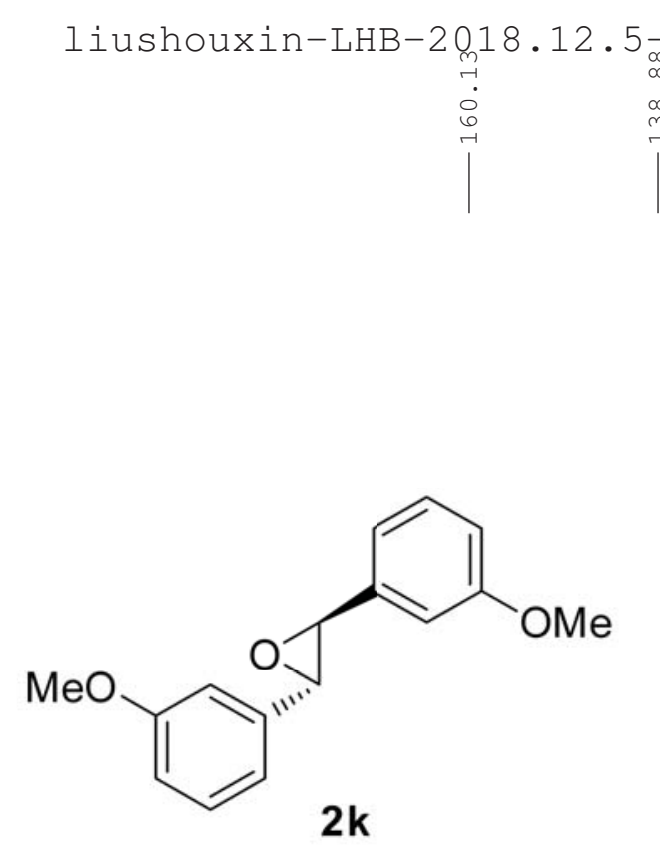

Bruker Avance III-500

$125 \mathrm{MHz}, \mathrm{CDCl}_{3}$
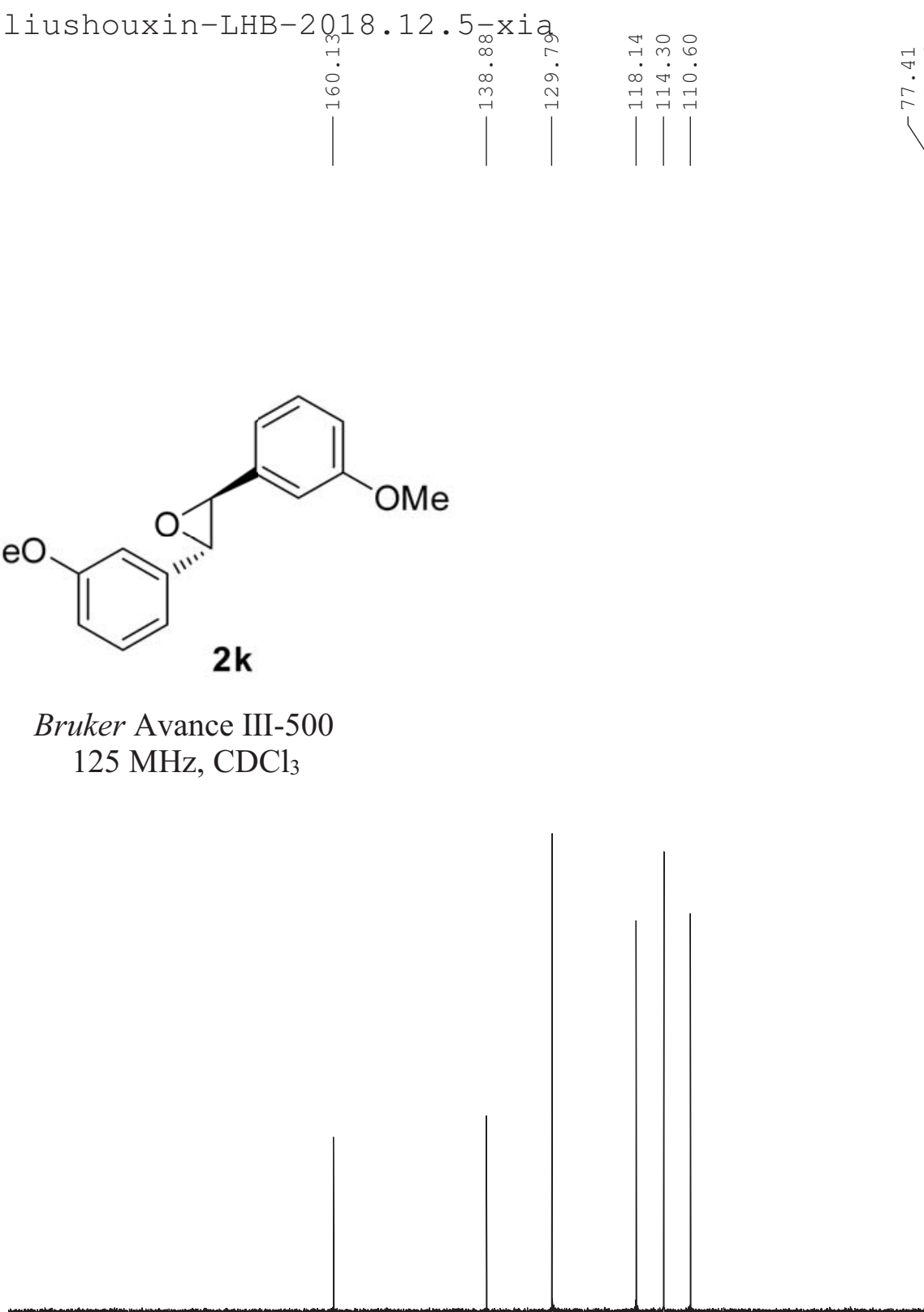

Brixter $(><)$

NAME
EXPNO

PROCNO

2018.12 .06

Time-

INSTRUM

PROBHD

PULPROG

TD

SOLVENT

NS
DS

SWH

$\begin{array}{lr}\text { SWH } & 29761.904 \mathrm{~Hz} \\ \text { FIDRES } & 0.908261 \mathrm{~Hz}\end{array}$

PIDRES $0.908261 \mathrm{~Hz}$

${ }_{\mathrm{R} G}^{\mathrm{AQ}}$

$\mathrm{DW}$

DW
DE
TE

$\mathrm{TE}$
$\mathrm{D} 1$

D11
TD 0

20181206

21.24

$5 \mathrm{~mm}$ PADUL 13C

zgpg 30
32768

$\mathrm{CDCl} 3$

$0.5505524 \mathrm{sec}$
1820

16.800 usec

6.50 usec

$.00000000 \mathrm{sec}$

$0.03000000 \mathrm{sec}$

- CHANNEL

NUC1

PL1

PL1W
SEO1

$======$

$13 \mathrm{C}$

$4.50 \mathrm{~dB}$

$33.60015869 \mathrm{~W}$

$125.7703643 \mathrm{MHz}$

$=======$ CHANNEL $\mathrm{f} 2 \mathrm{2}========$

CPDPRG2 waltz16

NUC2

$1 \mathrm{H}$

PL2

$\mathrm{PL} 12$

$\mathrm{PL} 13$

PL2W

PLI2W

PL13W

$\mathrm{SFO} 2$

SI

SF
WDW

WDW
SSB

SSB
LB

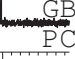

$0 \mathrm{ppm}$
80.00 usec

$2.00 \mathrm{~dB}$

$17.46 \mathrm{~dB}$

$17.46 \mathrm{~dB}$

$16.79986763 \mathrm{~W}$

$0.47786582 \mathrm{~W}$

$0.47786582 \mathrm{~W}$

$500.1320005 \mathrm{MHz}$

$125.7577738 \mathrm{MHz}$

$\mathrm{EM}$
0
$1.00 \mathrm{~Hz}$
0
1.40 


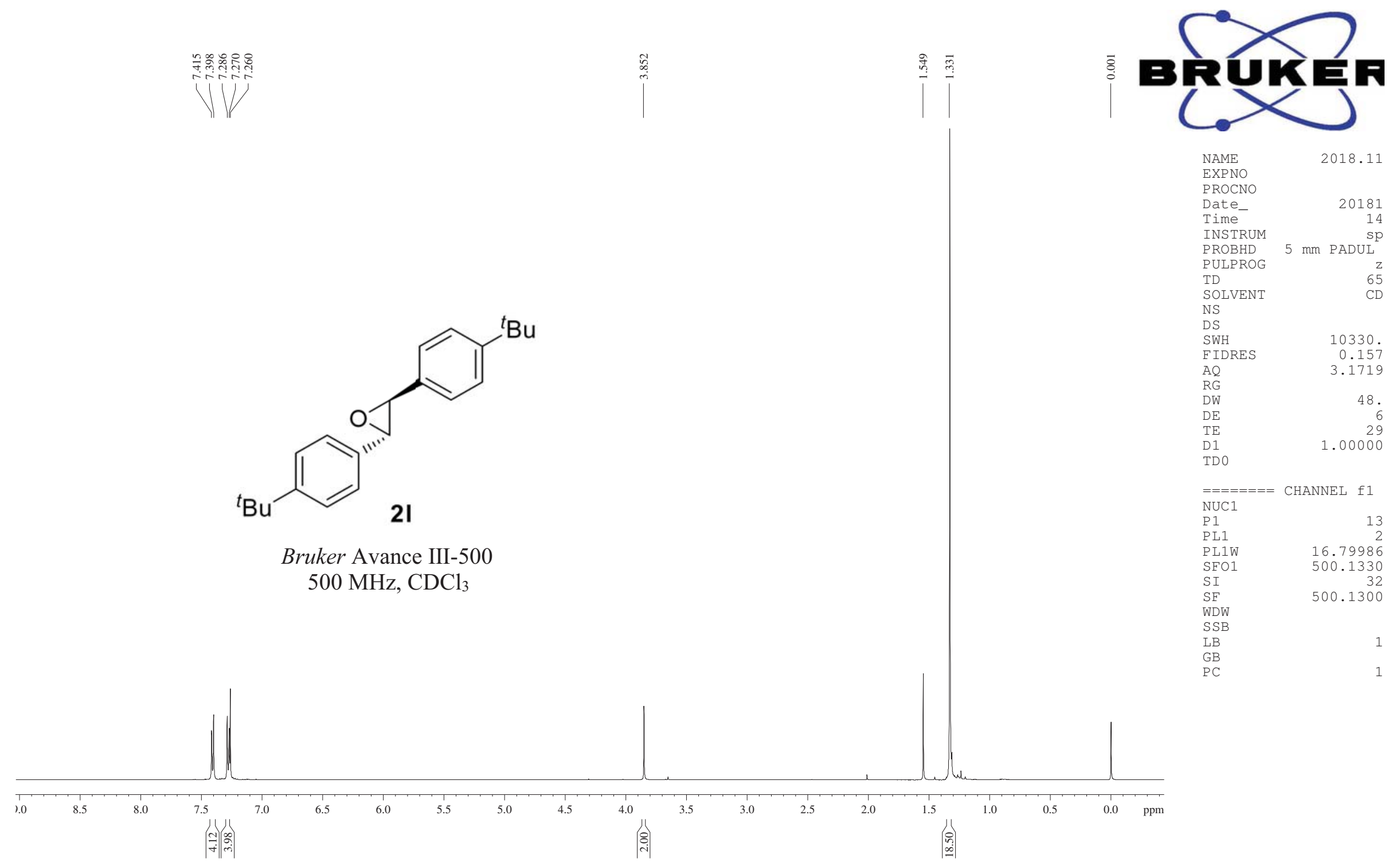




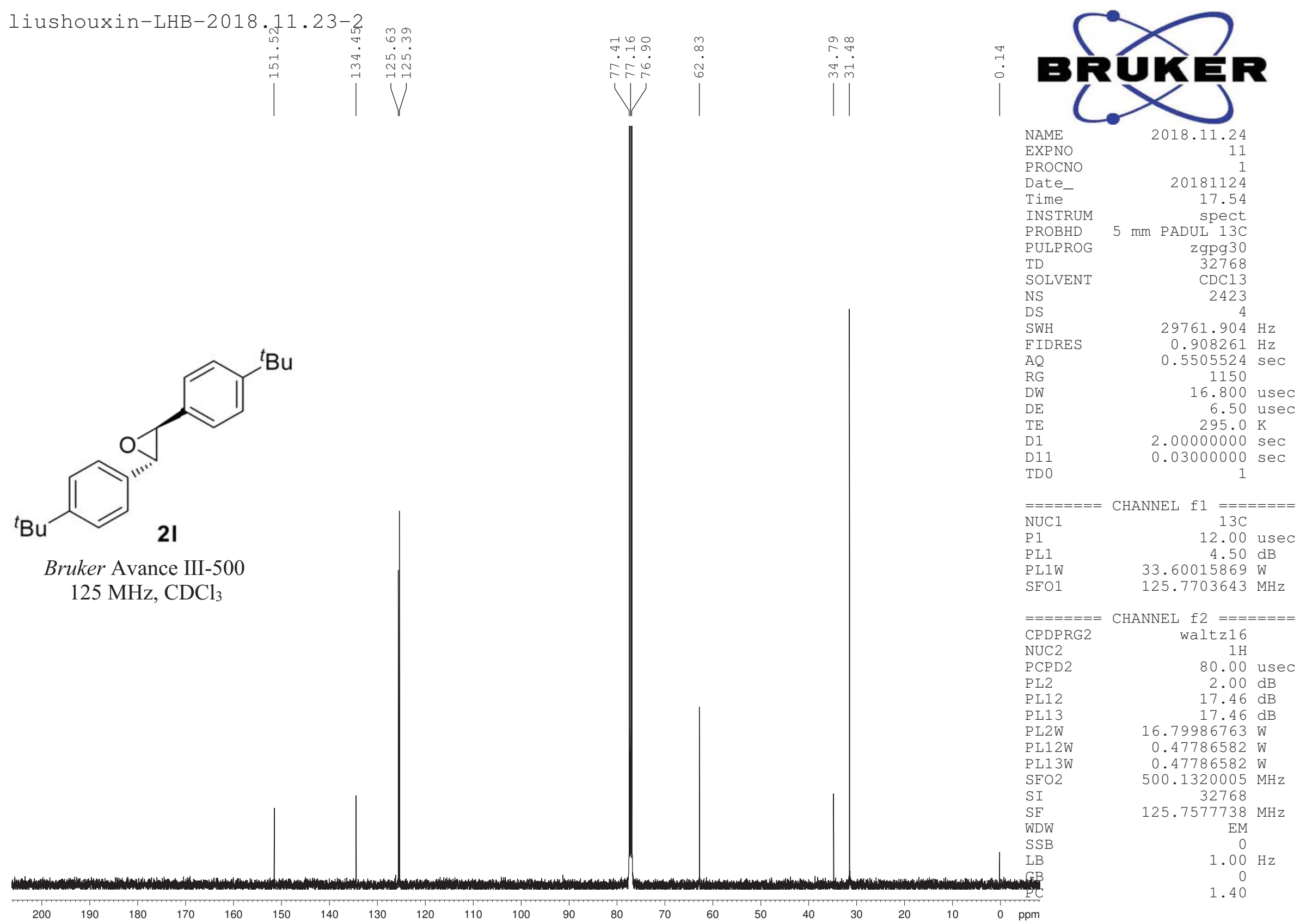




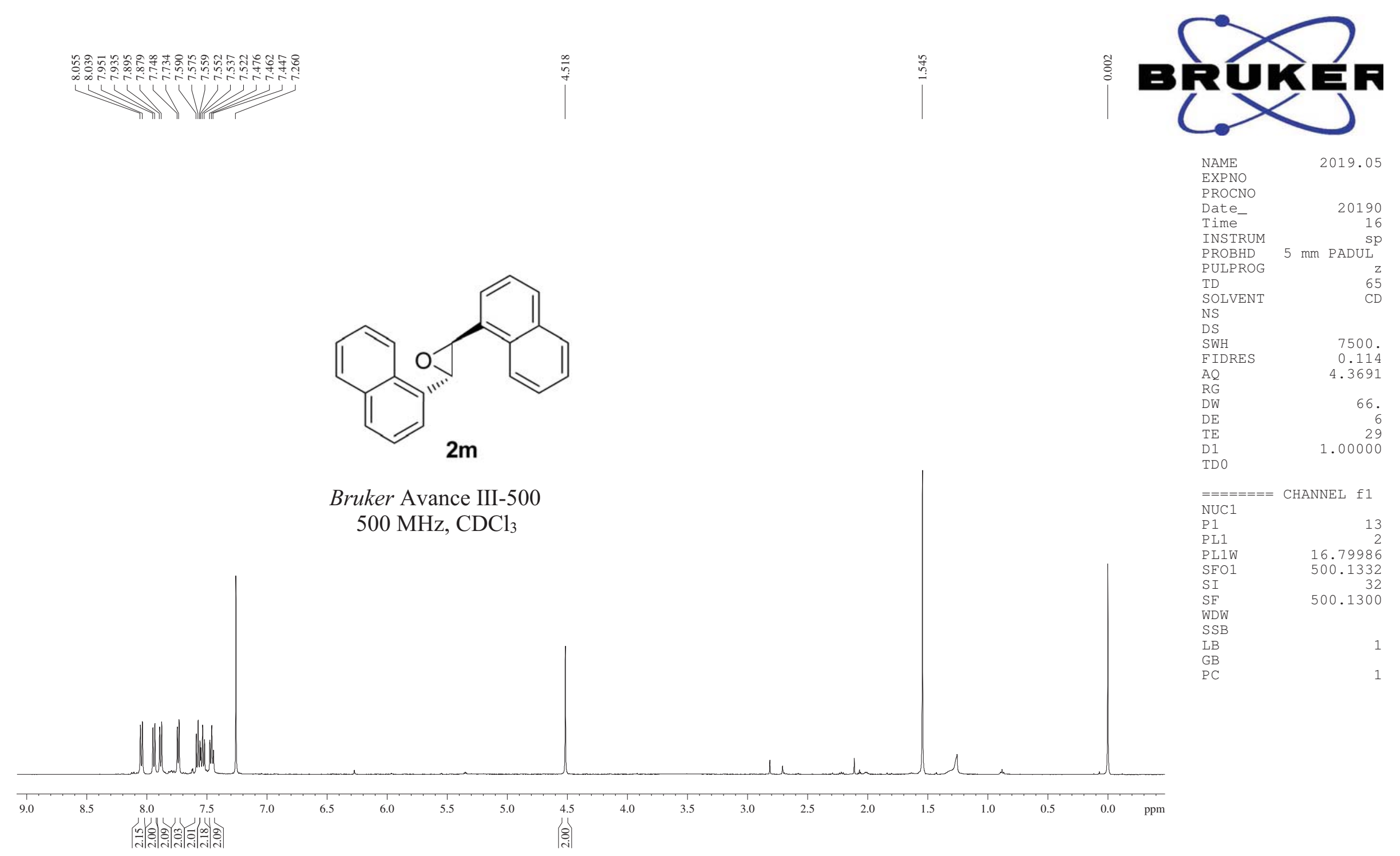




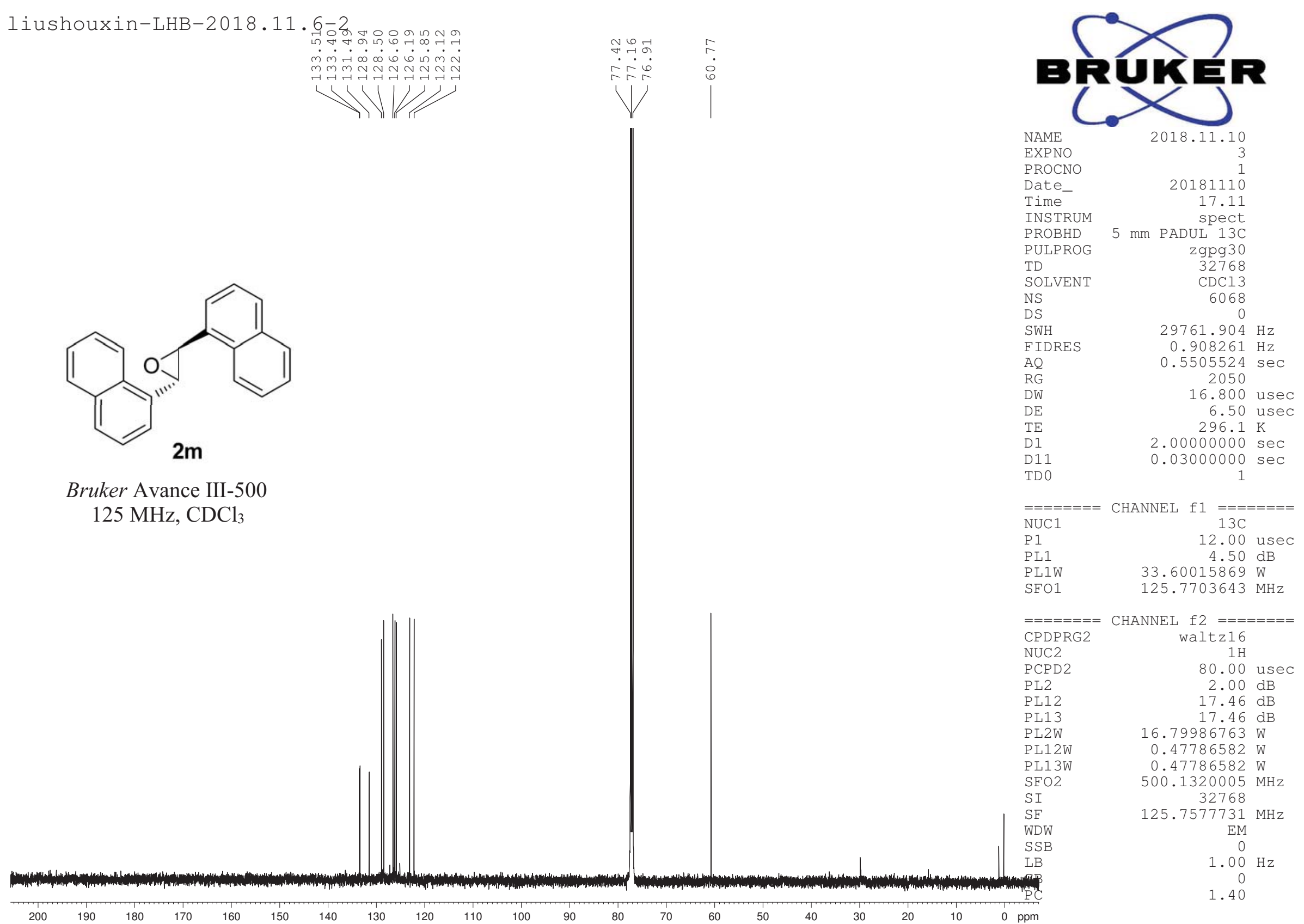




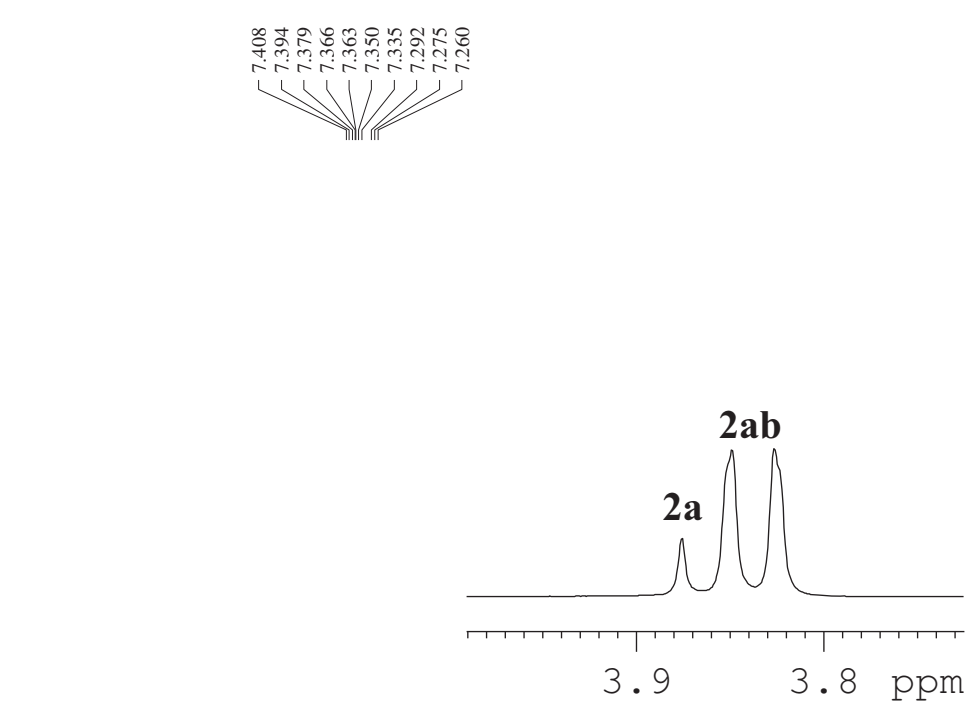

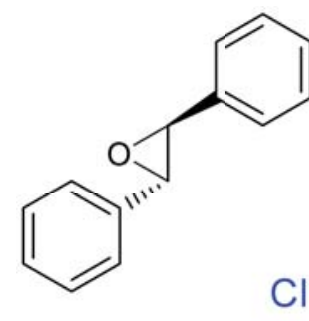

$2 a$ Bruker Avance III-500 $500 \mathrm{MHz}, \mathrm{CDCl}_{3}$

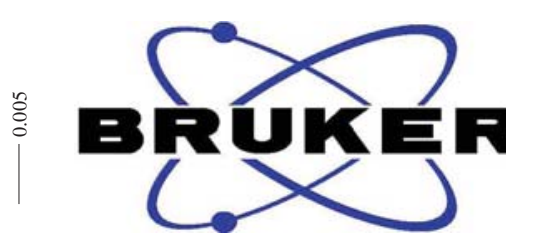

NAME

EXPNO

PROCNO

Date_-
Time

INSTRUM

PROBHD

PULPROG
TD

SOLVENT

NS

SWH

FIDRES

$\mathrm{AQ}$
$\mathrm{RG}$

RG

DE
TE

TE
D1
TD O

TDO

$=======$ CHANNEL $\mathrm{f} 1====$ NUC1

P1

$$
\text { PL }
$$

$$
\text { SF }
$$$$
\text { SI }
$$$$
\begin{aligned}
& S F \\
& \text { WDW }
\end{aligned}
$$$$
\begin{aligned}
& \text { WDW } \\
& \text { SSB }
\end{aligned}
$$$$
\begin{aligned}
& \mathrm{SSB} \\
& \mathrm{LB} \\
& \mathrm{GB}
\end{aligned}
$$$$
\text { GB }
$$

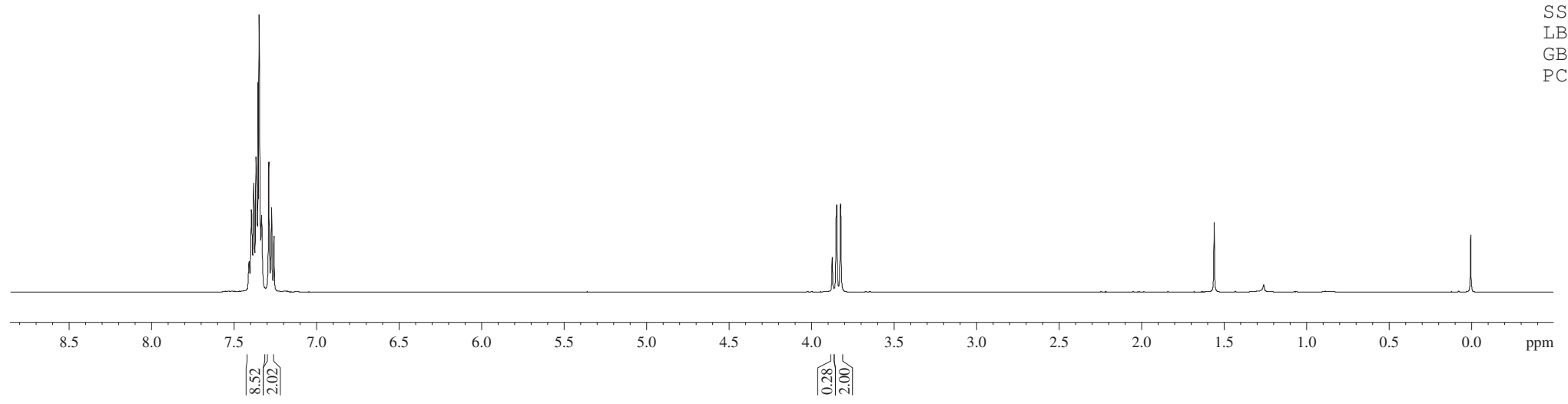




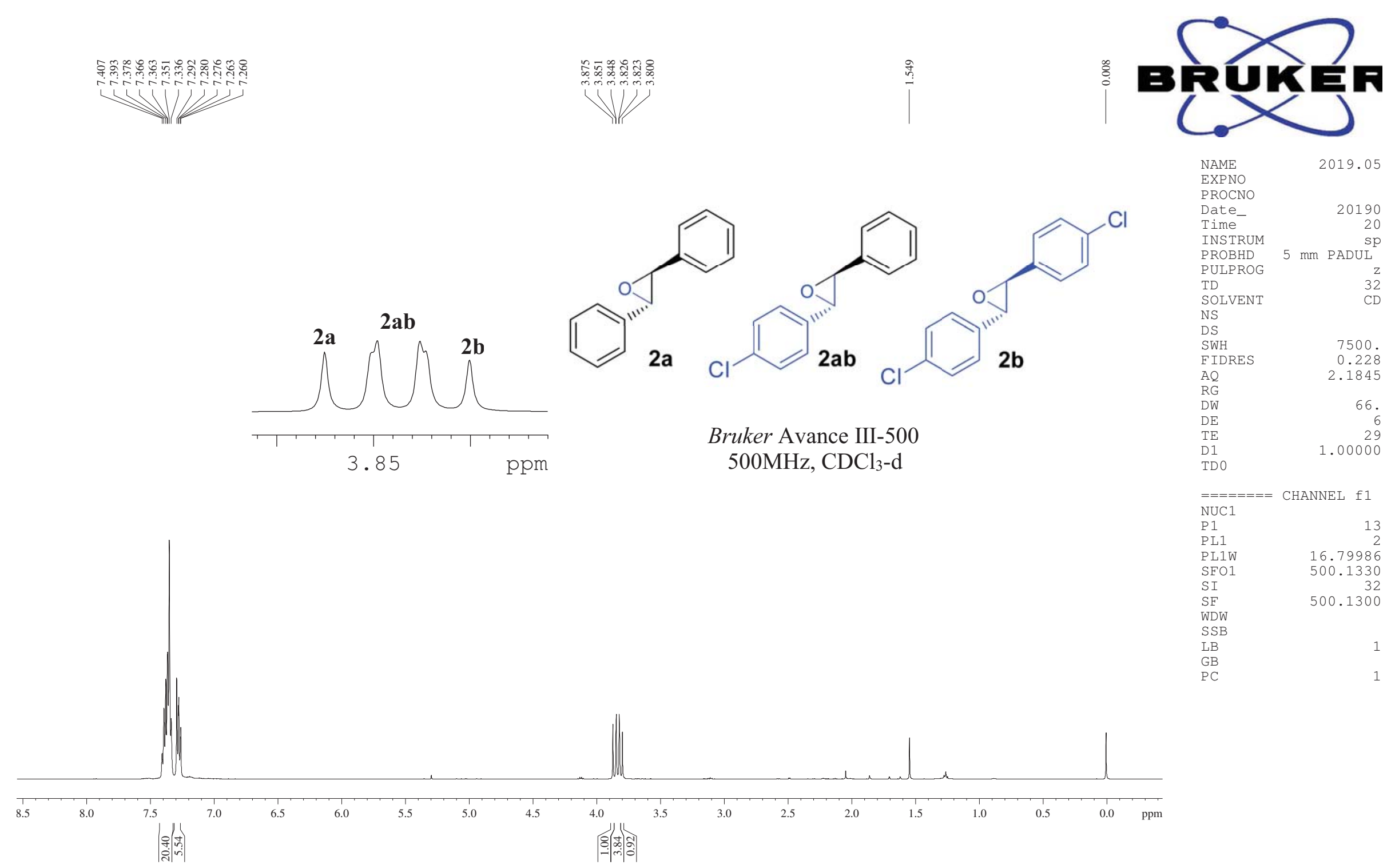




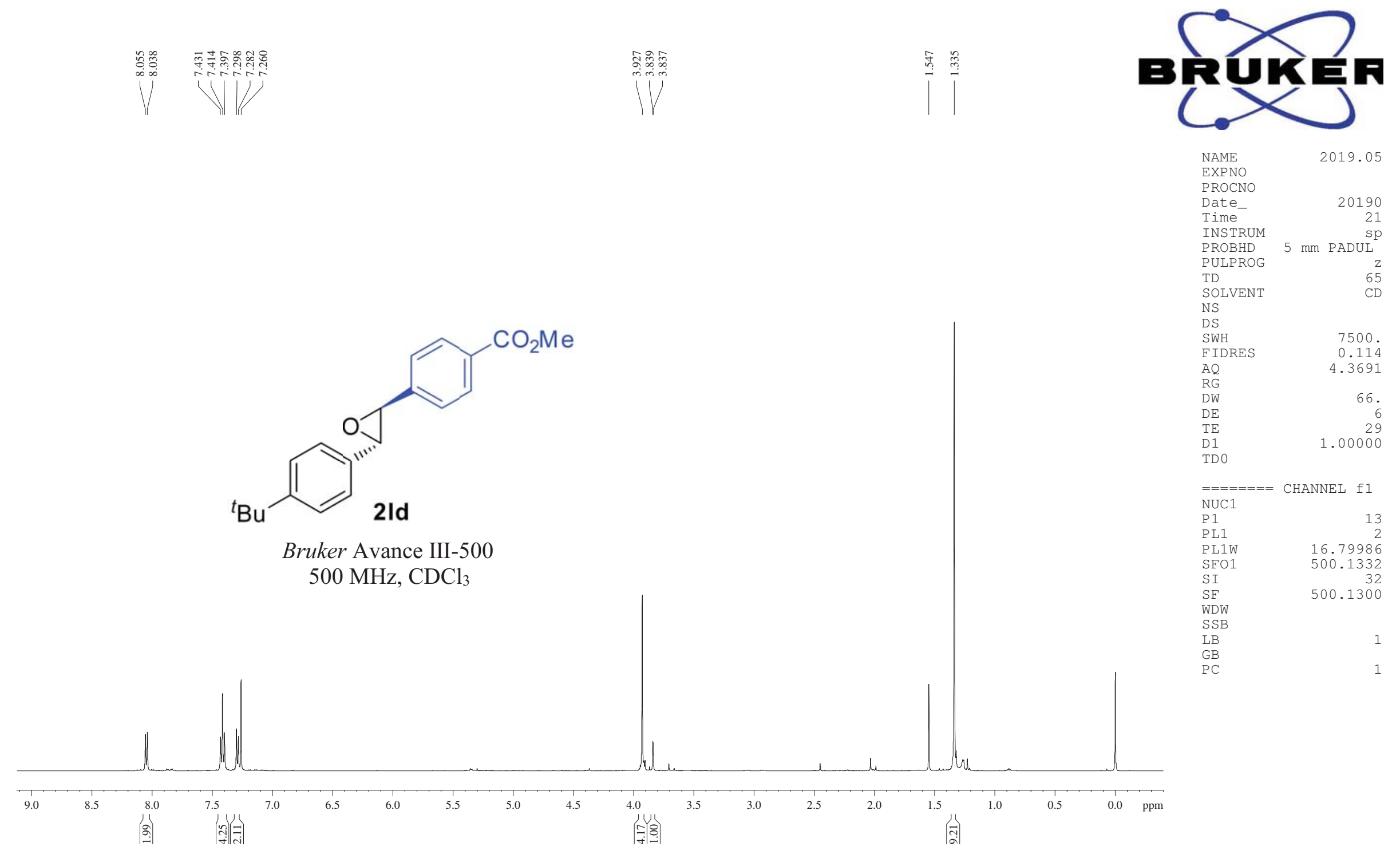




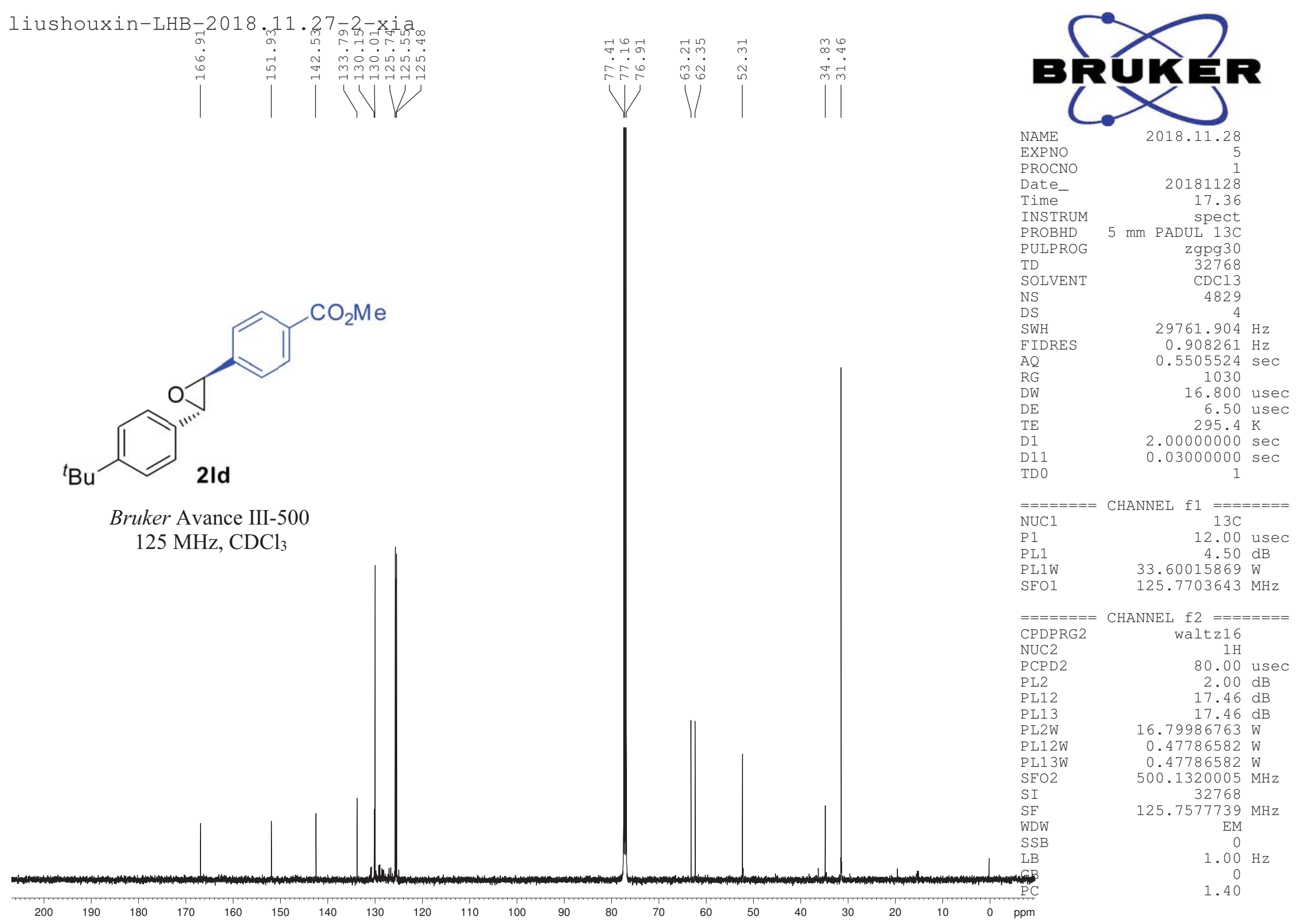




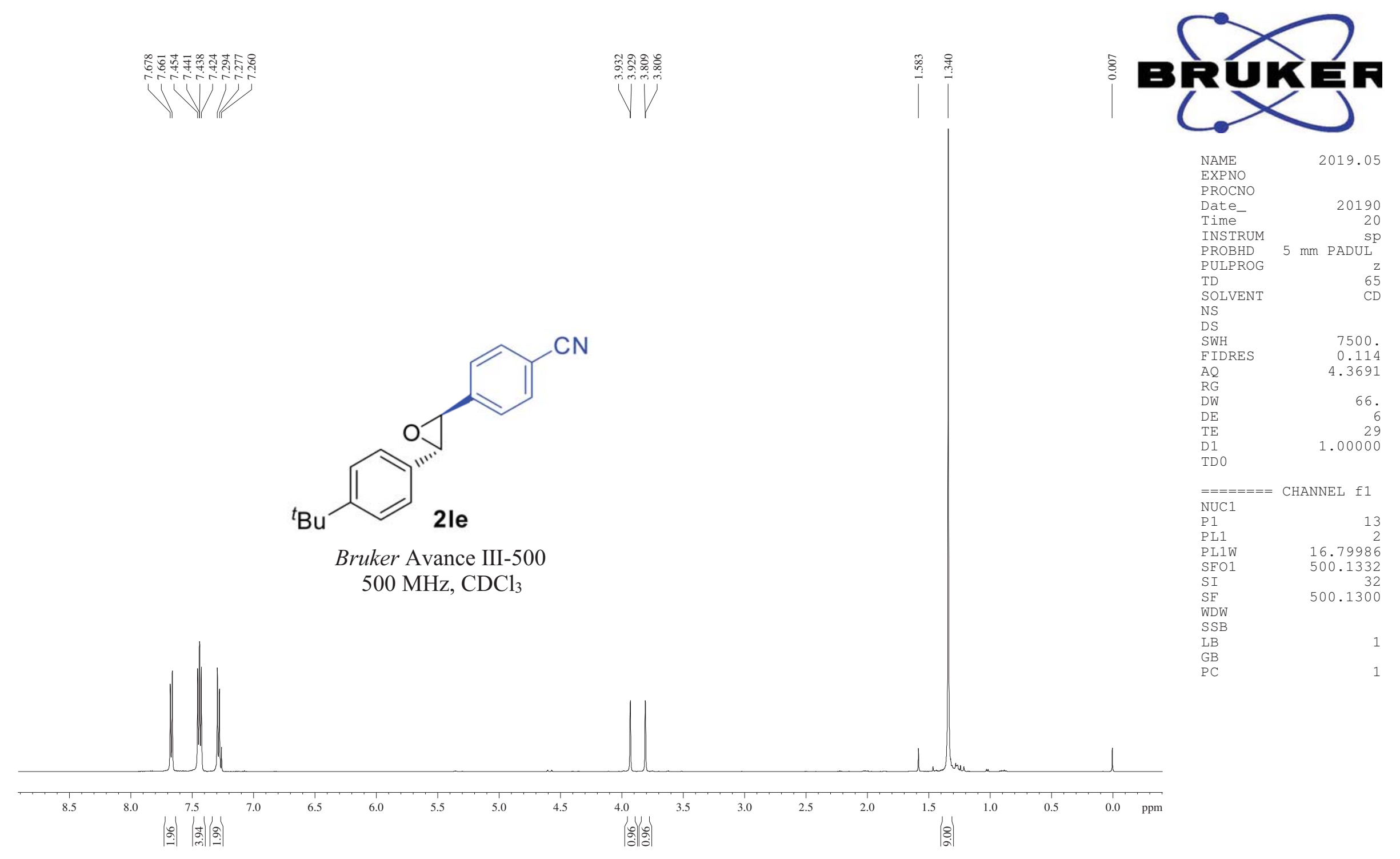




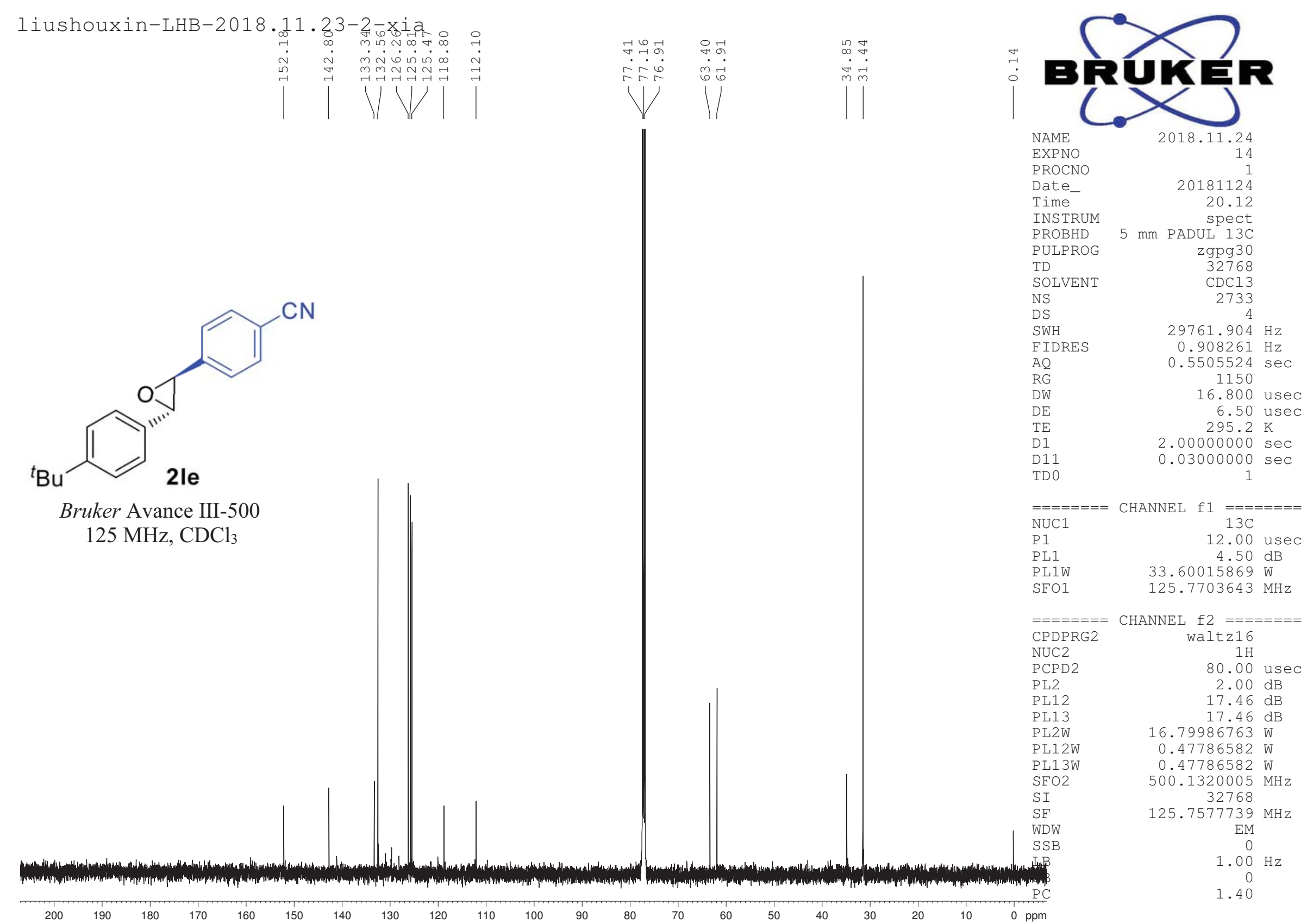




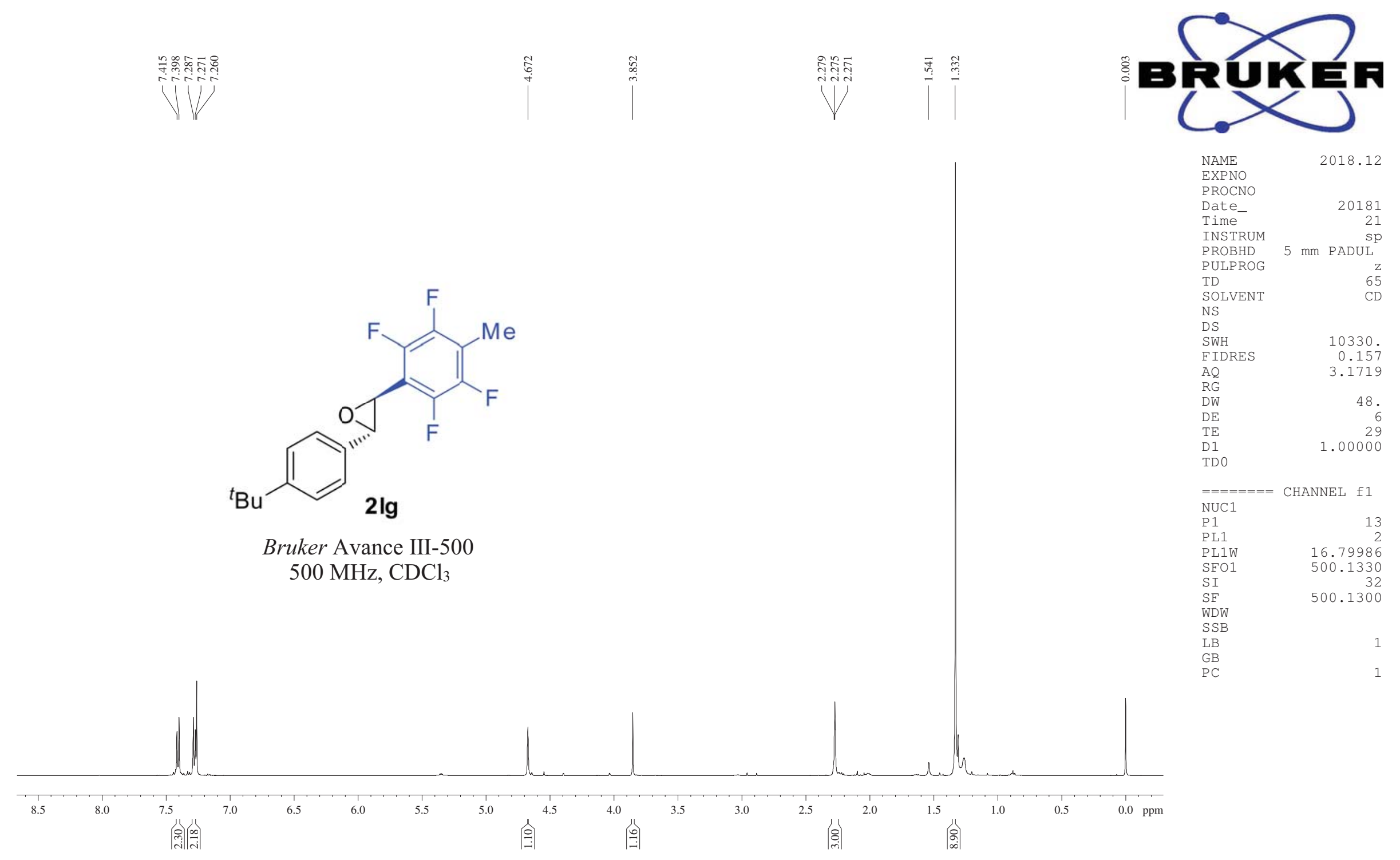




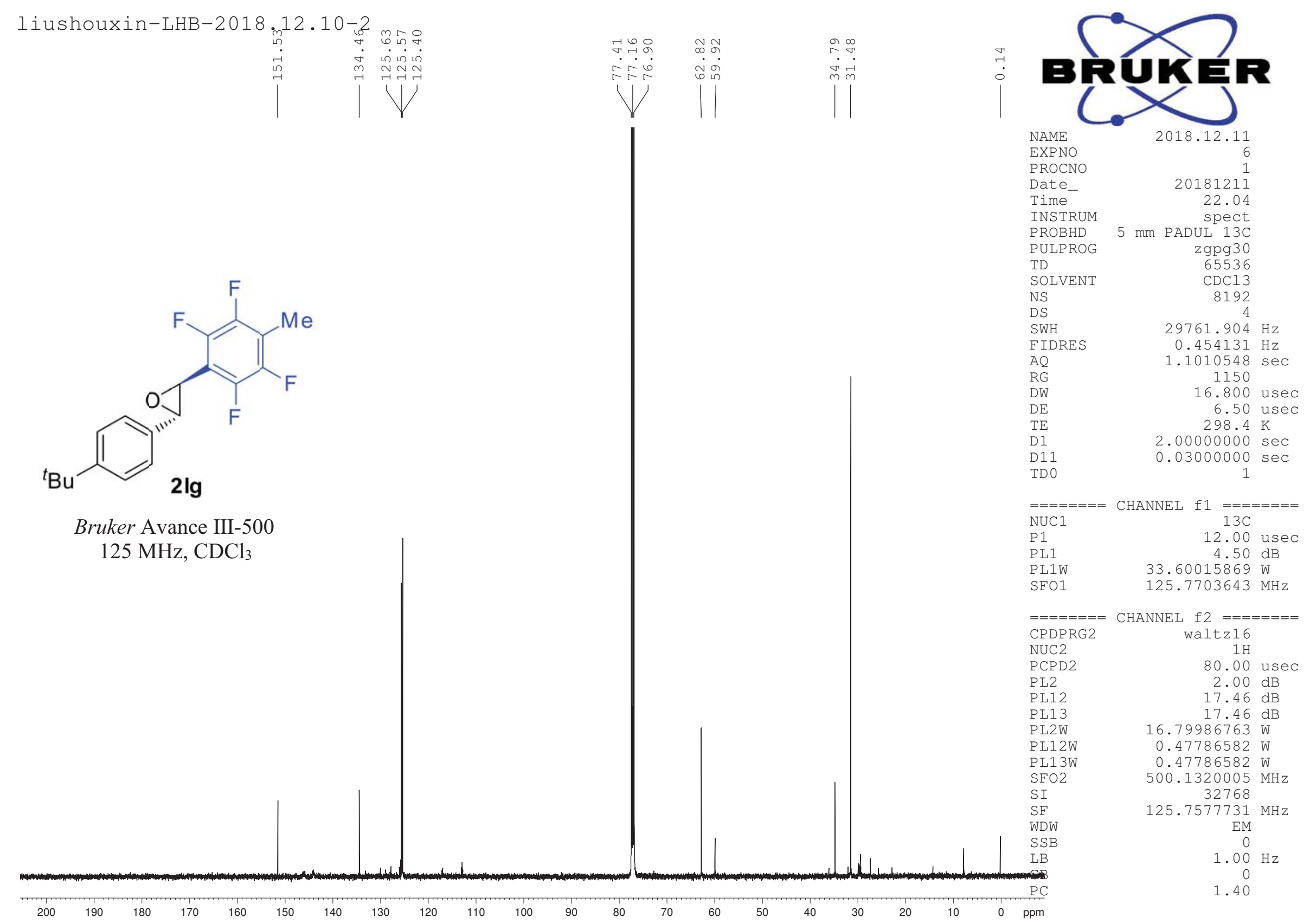




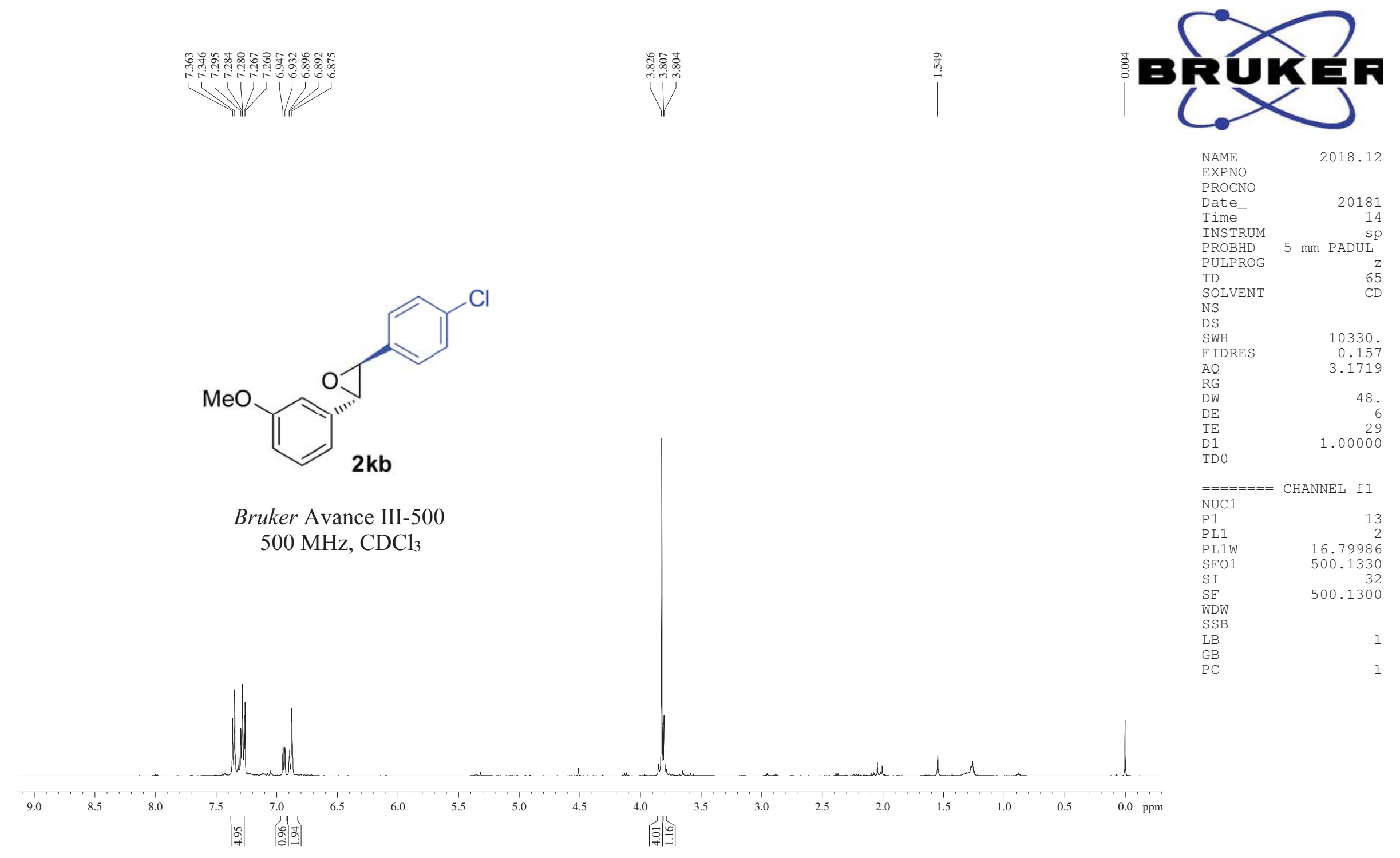




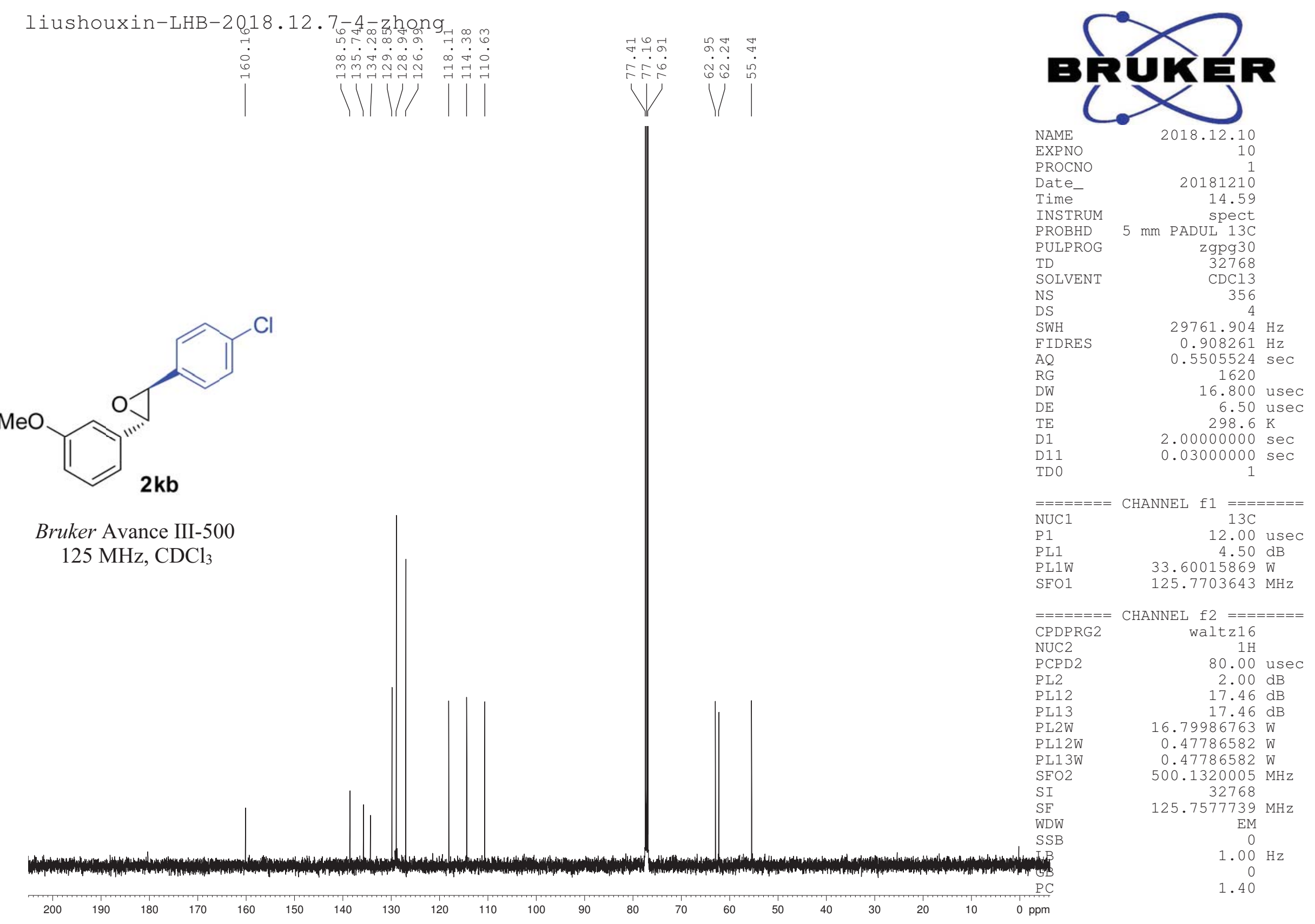




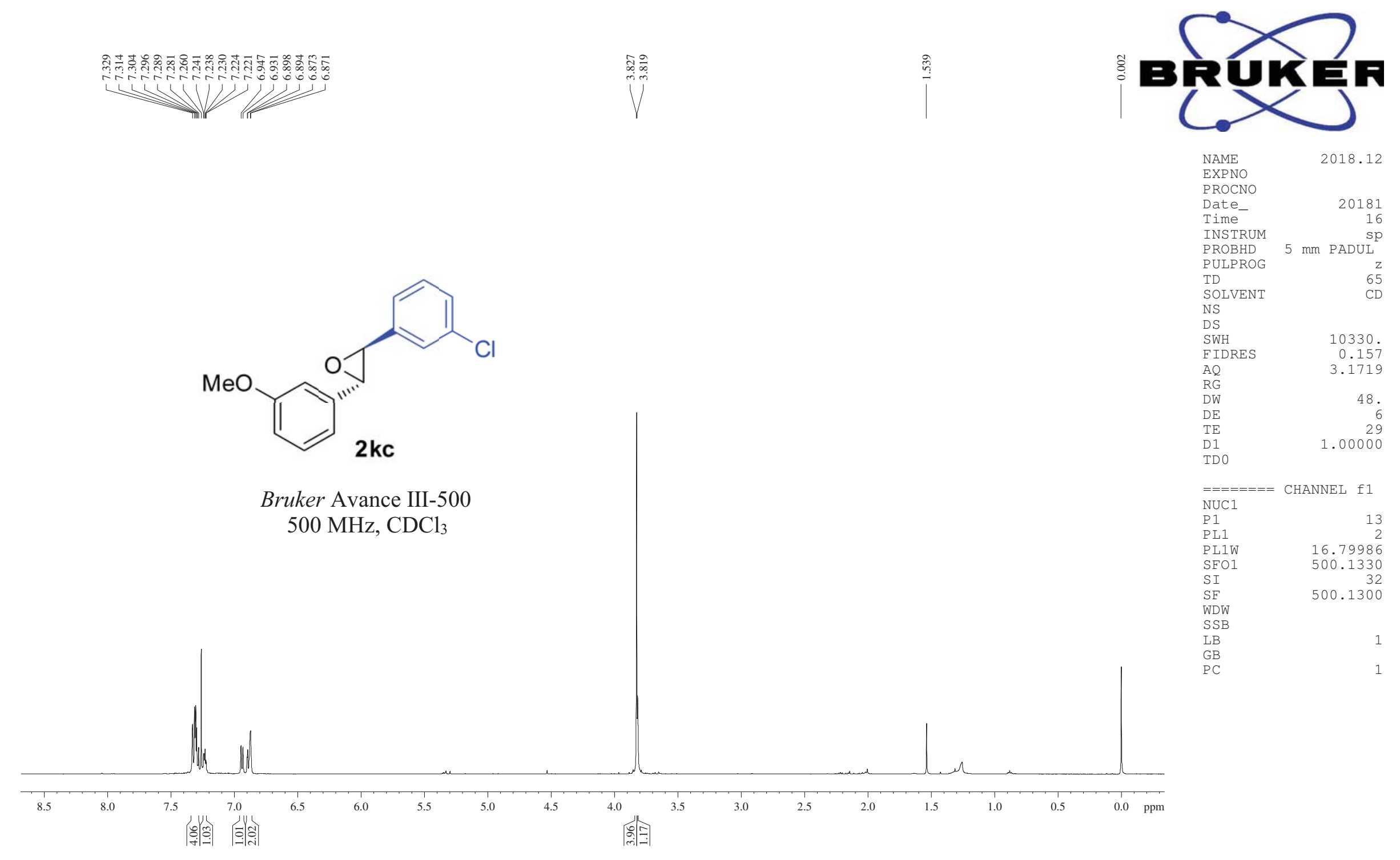



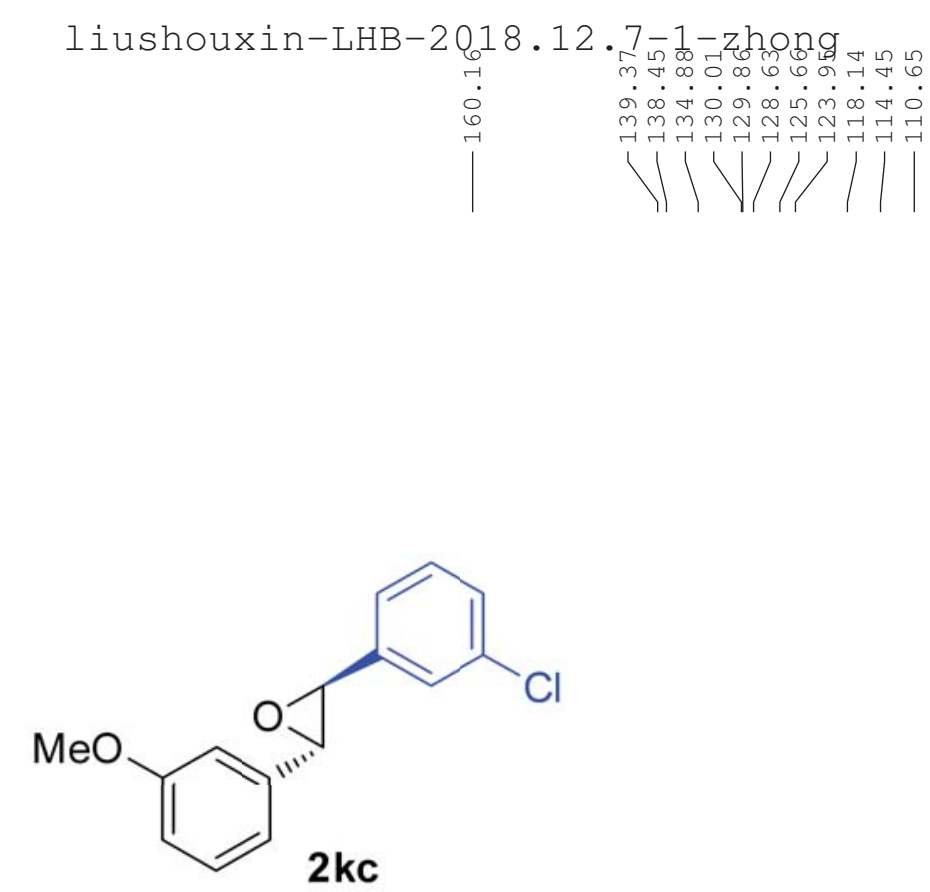

Bruker Avance III-500 $125 \mathrm{MHz}, \mathrm{CDCl}_{3}$

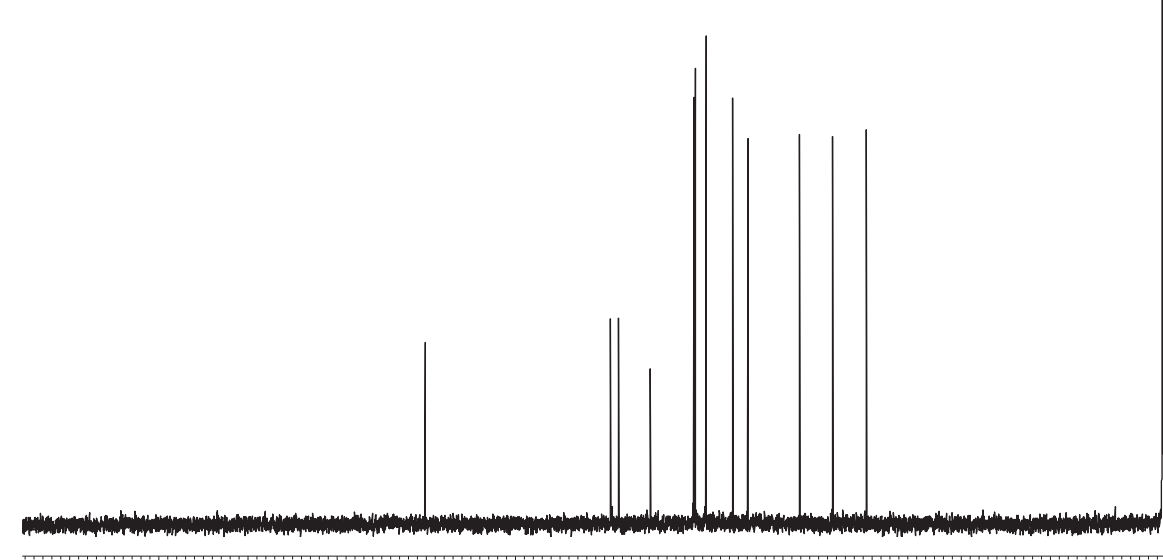

\section{1}

\section{(O)}

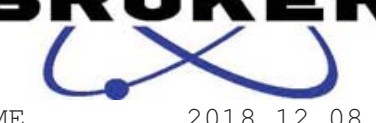

NAME

PROCNO

Date

Time-

PROBHD

PULPROG

TD

SOLVENT

NS

$\begin{array}{lr}\text { SWH } & 29761.904 \mathrm{~Hz} \\ \text { FIDRES } & 0.908261 \mathrm{~Hz}\end{array}$

AQ $\quad 0.5505524 \mathrm{sec}$

RG

DW

DE

D1

TDO

$=======$ CHANNEL $\mathrm{f}$

NUC1

PL1

PL1W
SFO1

20181208

17.05

$5 \mathrm{~mm}$ PADUL $13 \mathrm{C}$

zgpg 30

32768

6144

6.800 usec

$6.50 \mathrm{usec}$
$298.3 \mathrm{~K}$

$.00000000 \mathrm{sec}$

$0.03000000 \mathrm{sec}$

CPDPRG2

$\mathrm{NUC2}$

PL2

$\mathrm{PL} 12$

$\mathrm{PL} 13$

PL $2 \mathrm{~W}$

PL12W

PL13W

$\mathrm{SFO} 2$

$\mathrm{SI}$

SF

WDW

SSB
LB

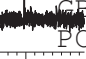

0 ppm
$=======$

$4.50 \mathrm{~dB}$

$33.60015869 \mathrm{~W}$

$125.7703643 \mathrm{MHz}$

CHANNEL f2 $========$

waltz16

80.00 use

$2.00 \mathrm{~dB}$

$17.46 \mathrm{~dB}$

$17.46 \mathrm{~dB}$

$16.79986763 \mathrm{~W}$

$0.47786582 \mathrm{~W}$

$0.47786582 \mathrm{~W}$

$500.1320005 \mathrm{MHz}$

$125.7577730 \mathrm{MHz}$

EM
0
$1.00 \mathrm{~Hz}$
0
1.40 


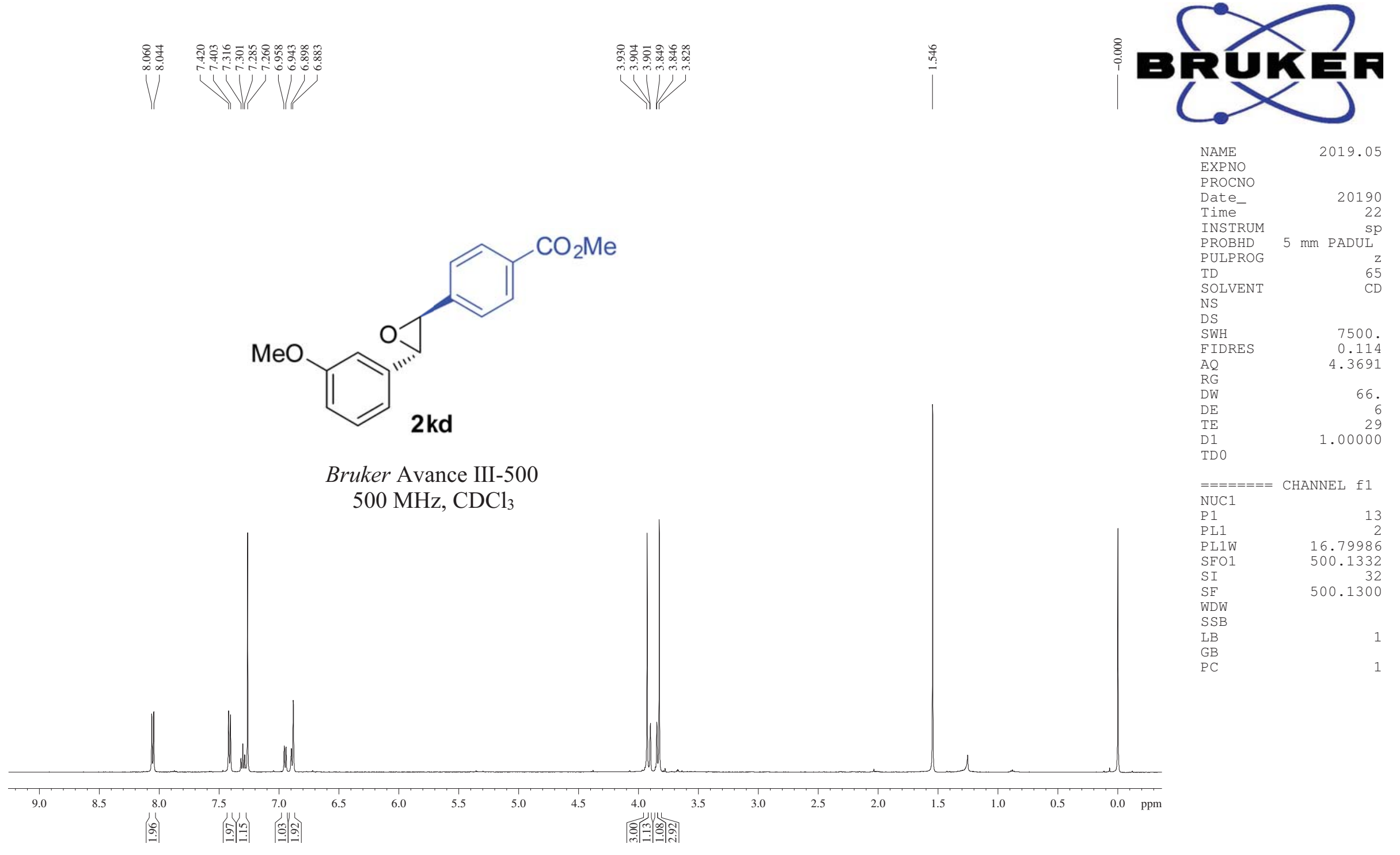




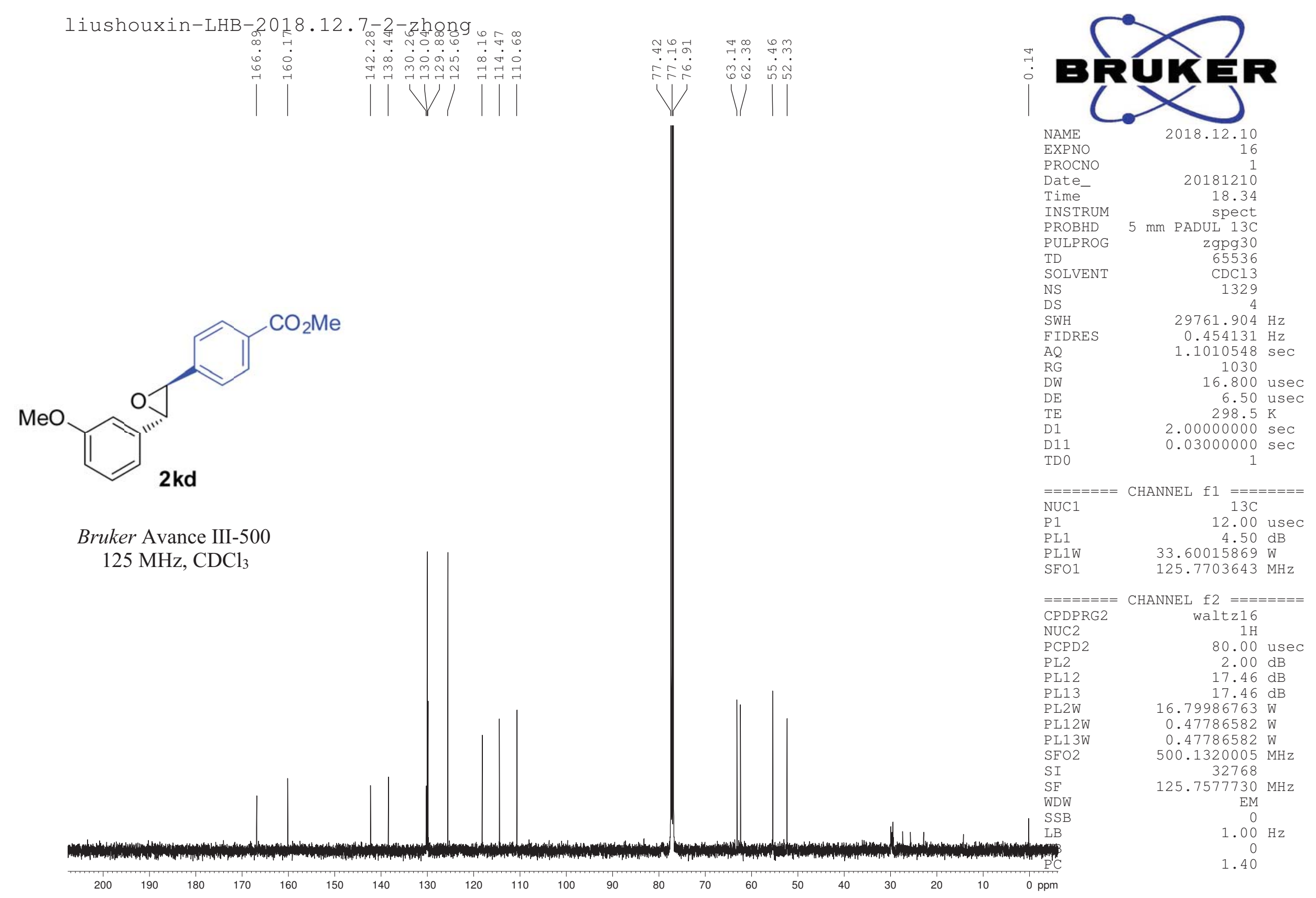




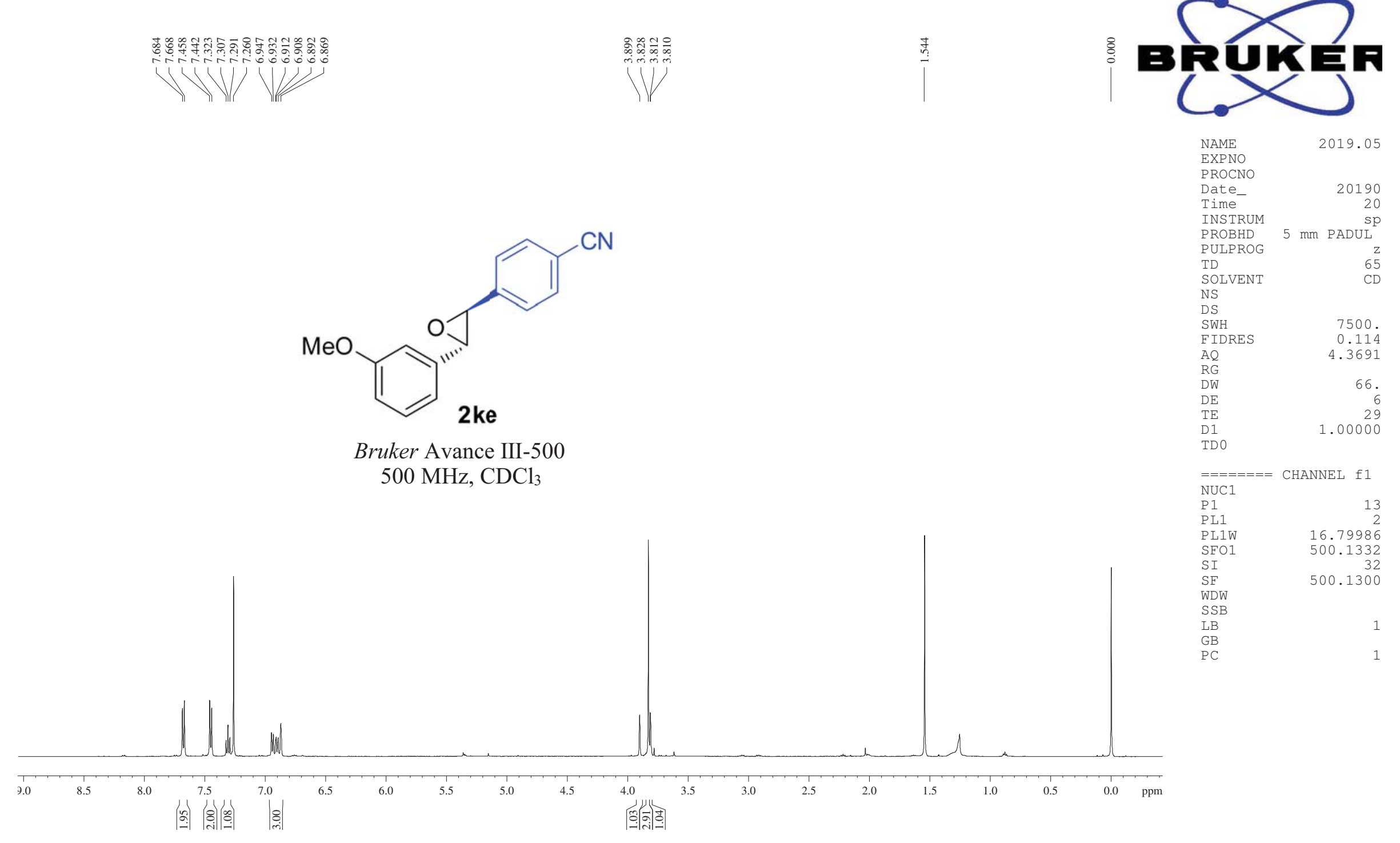




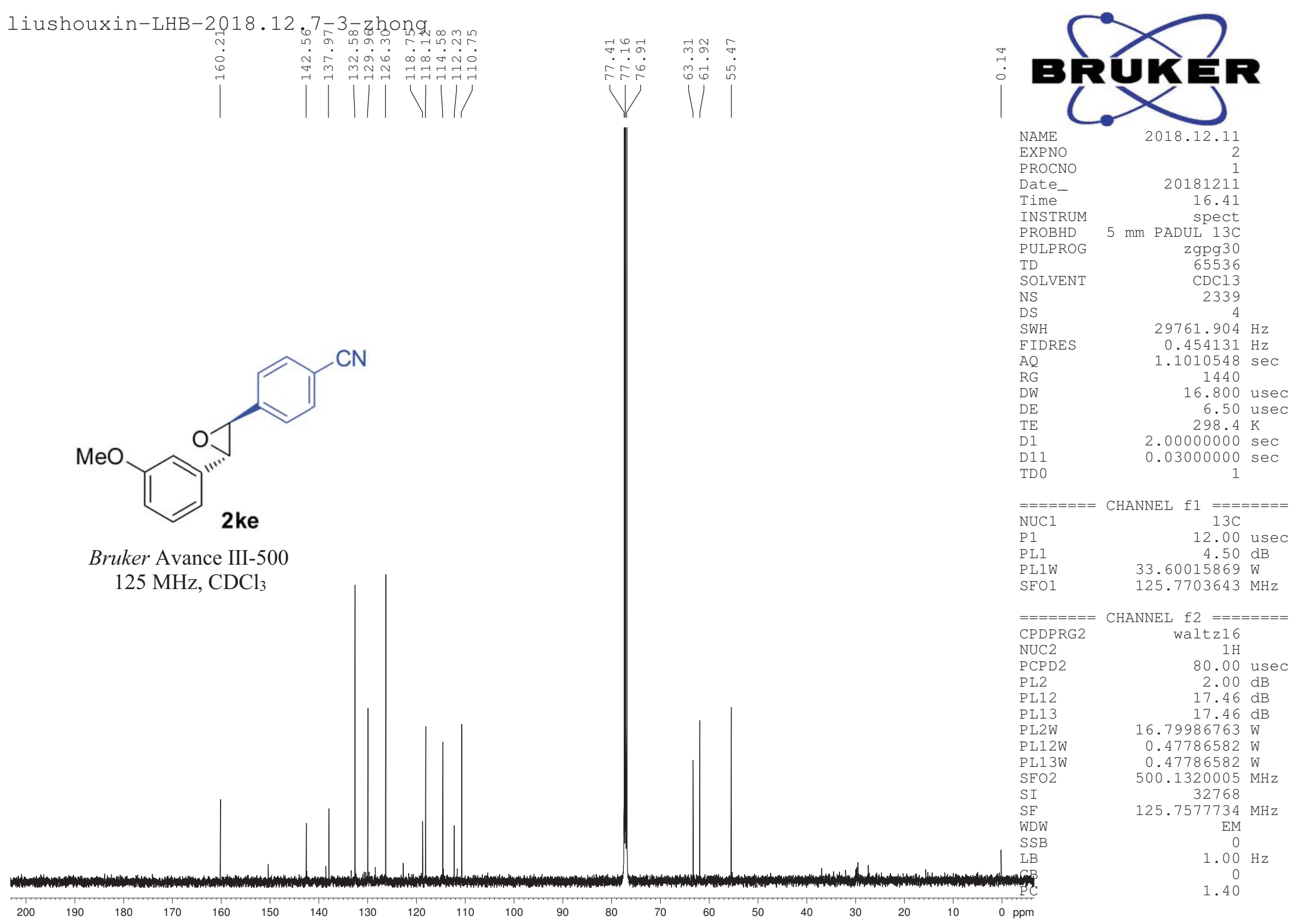




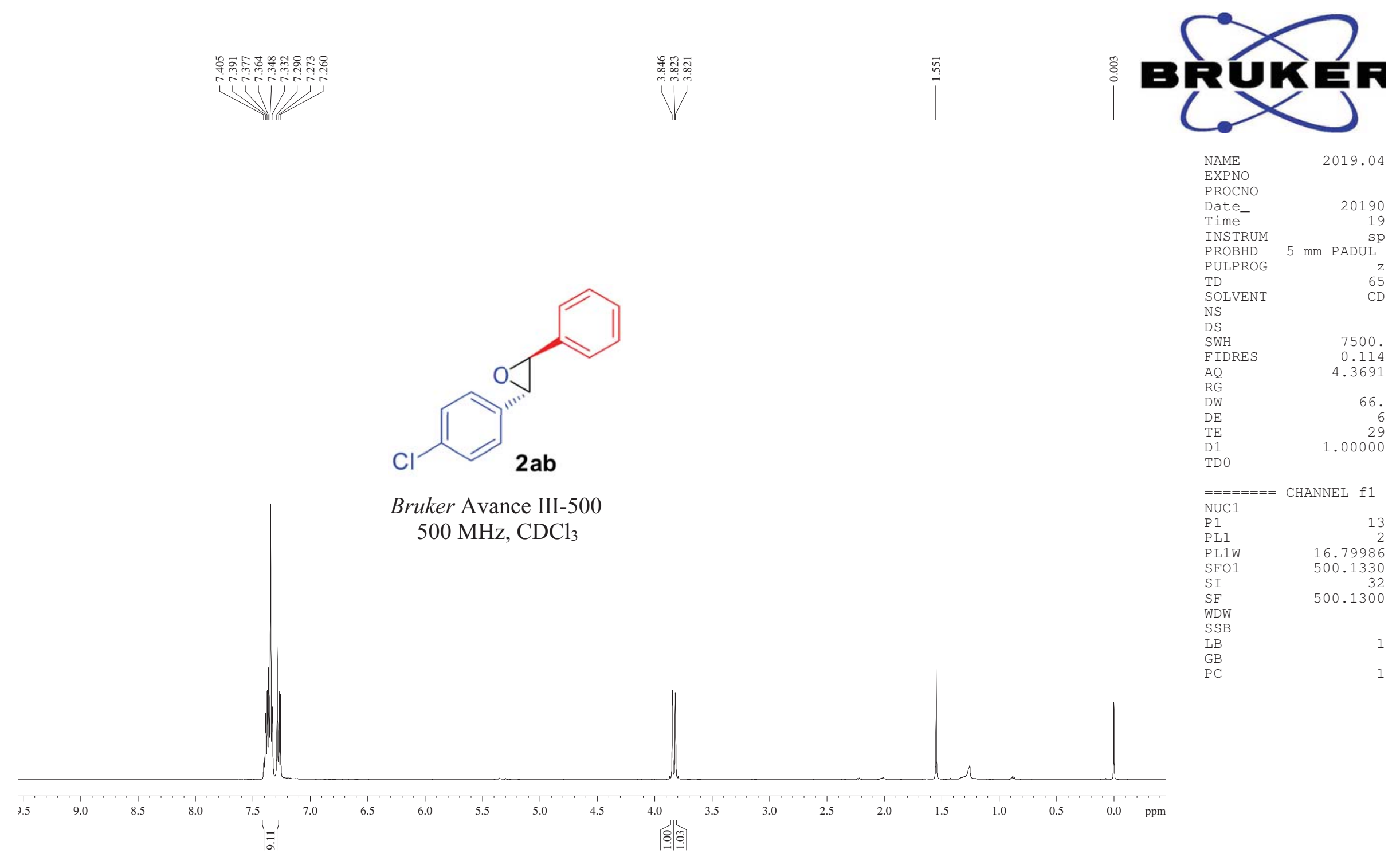




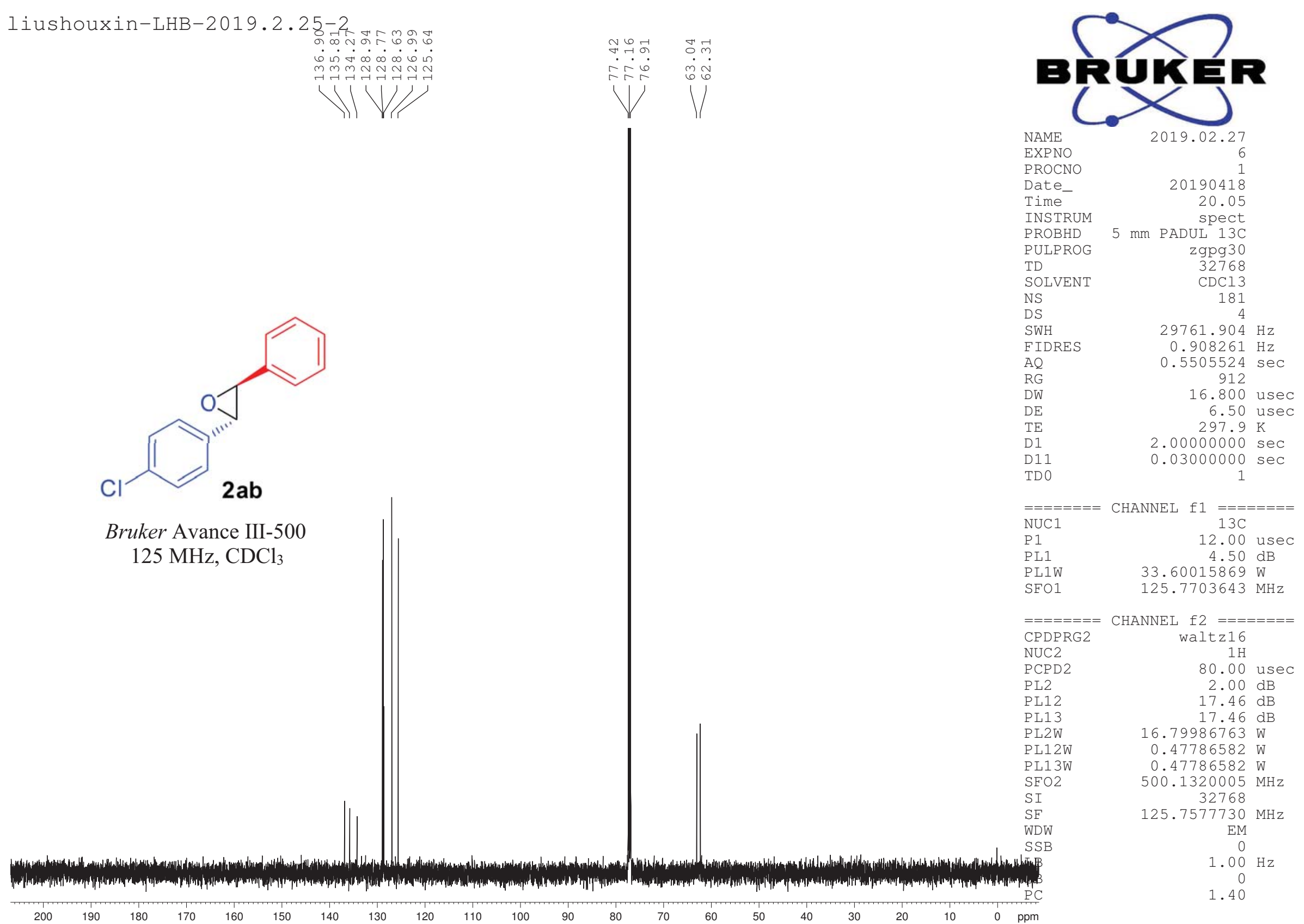




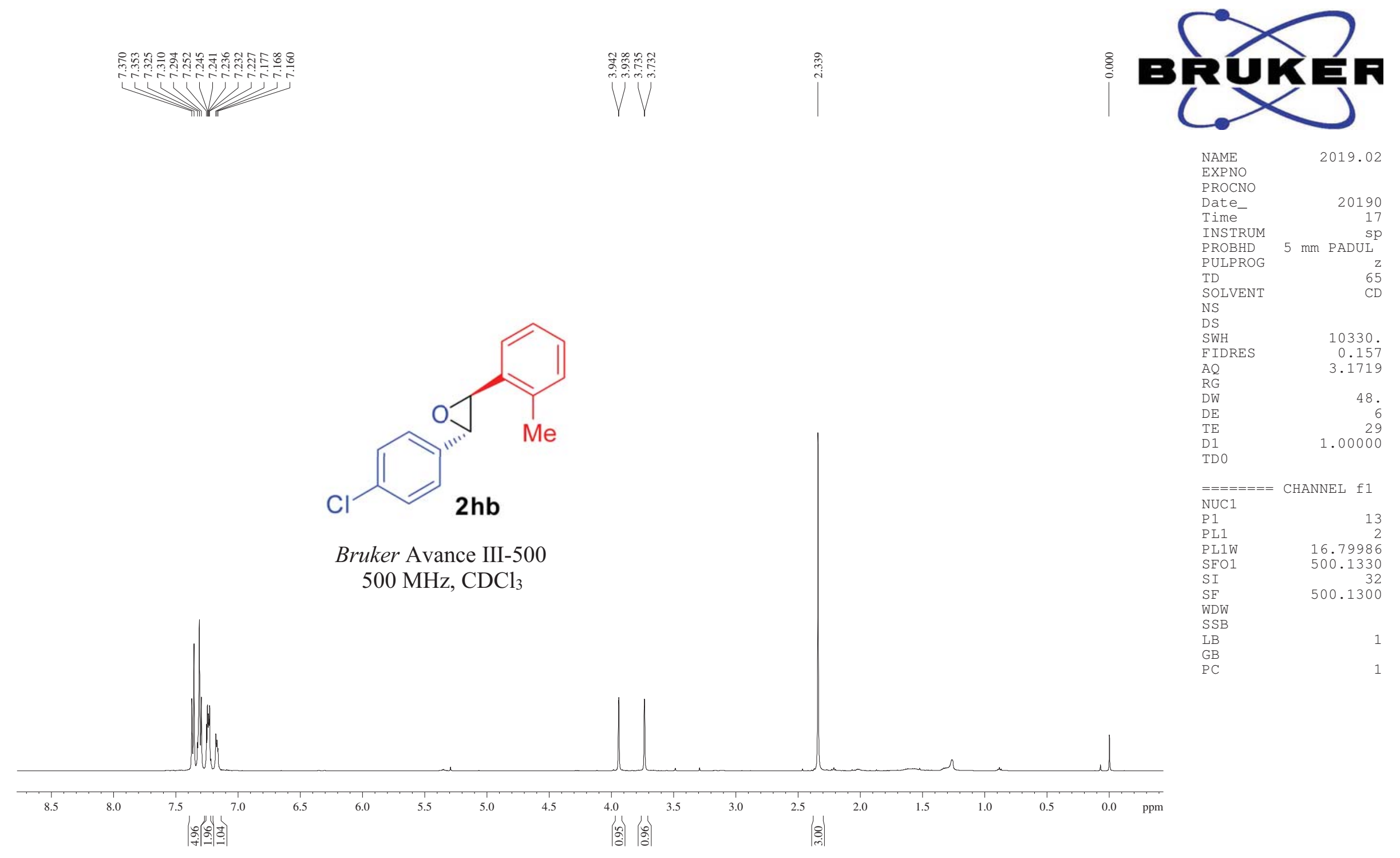




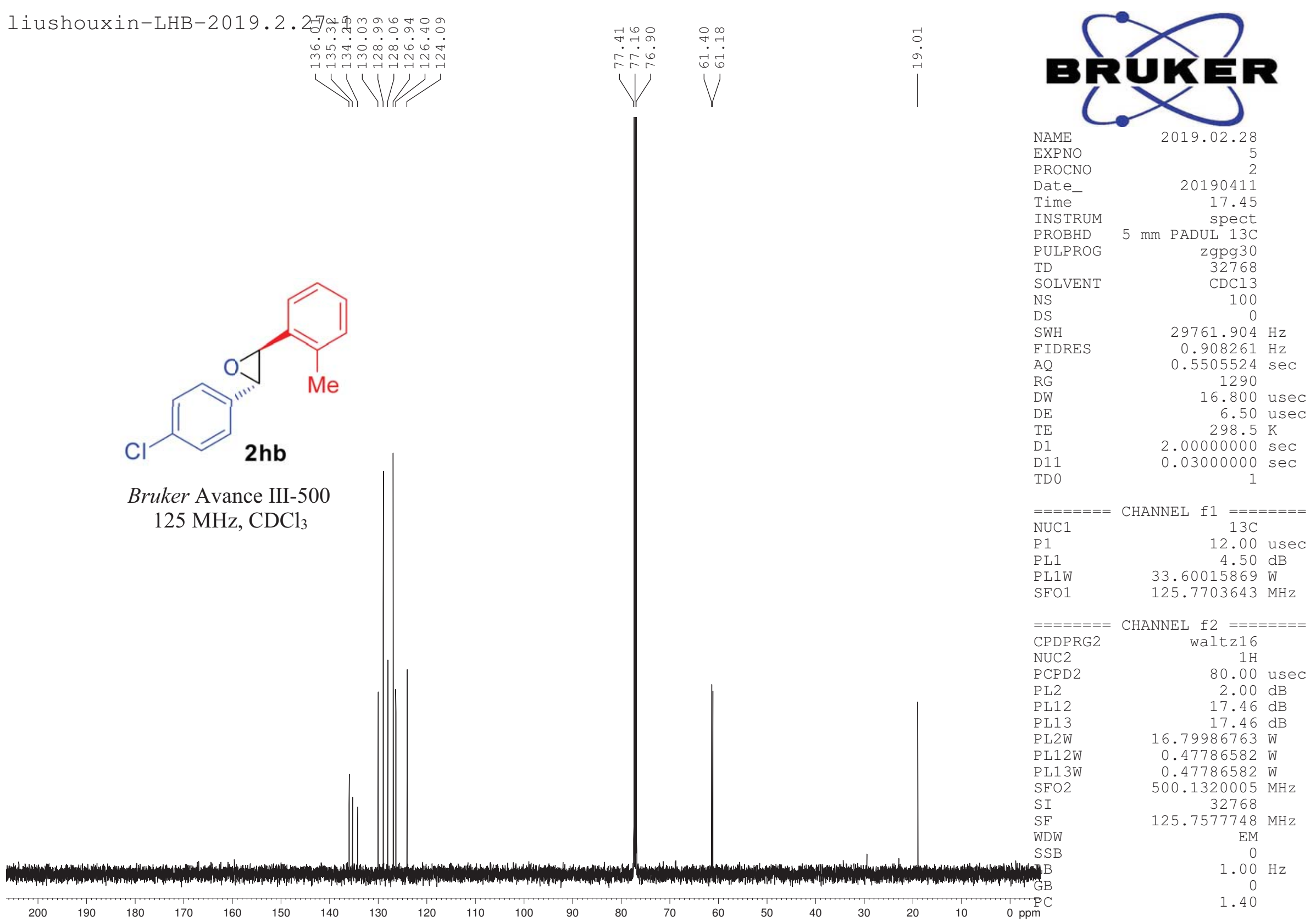




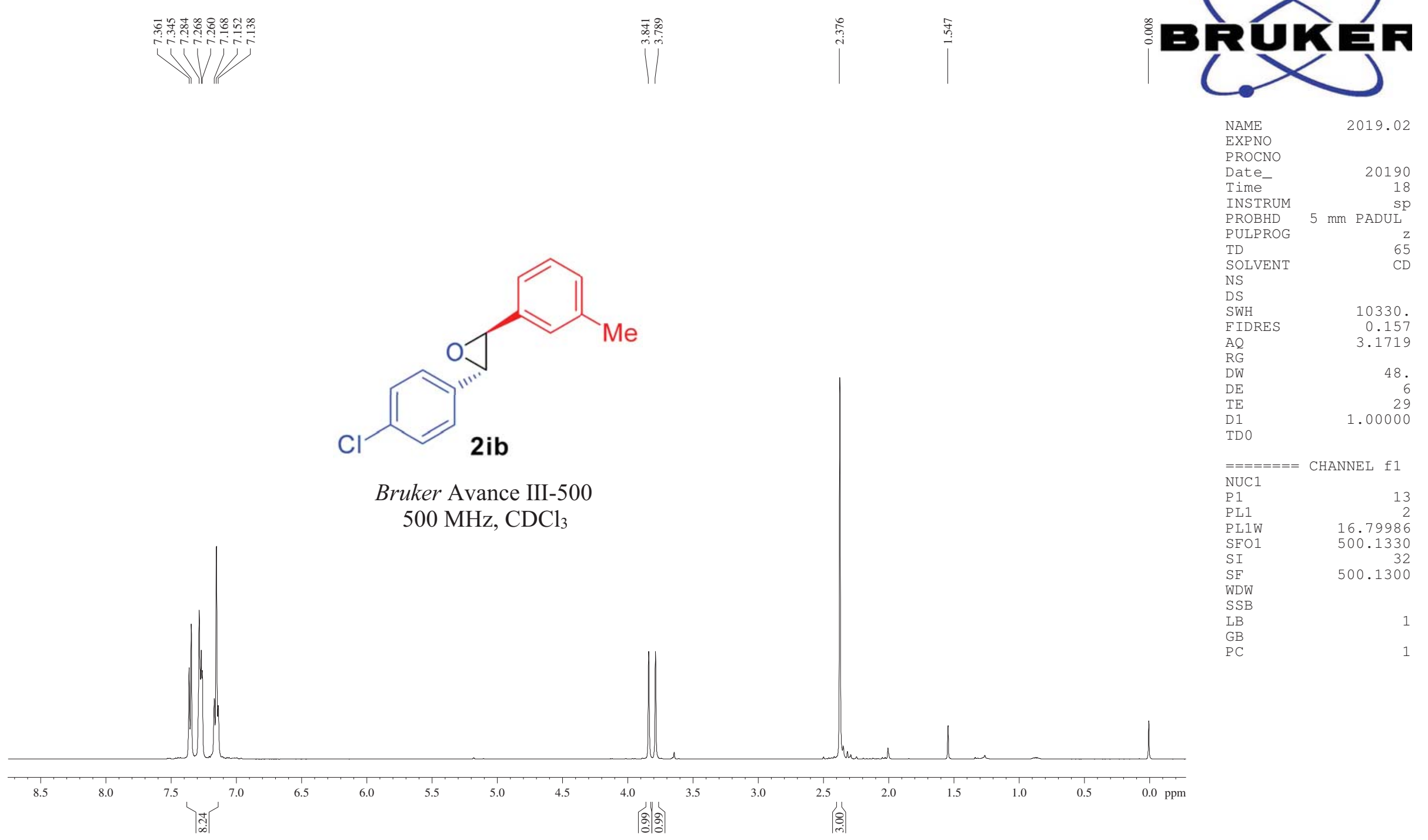




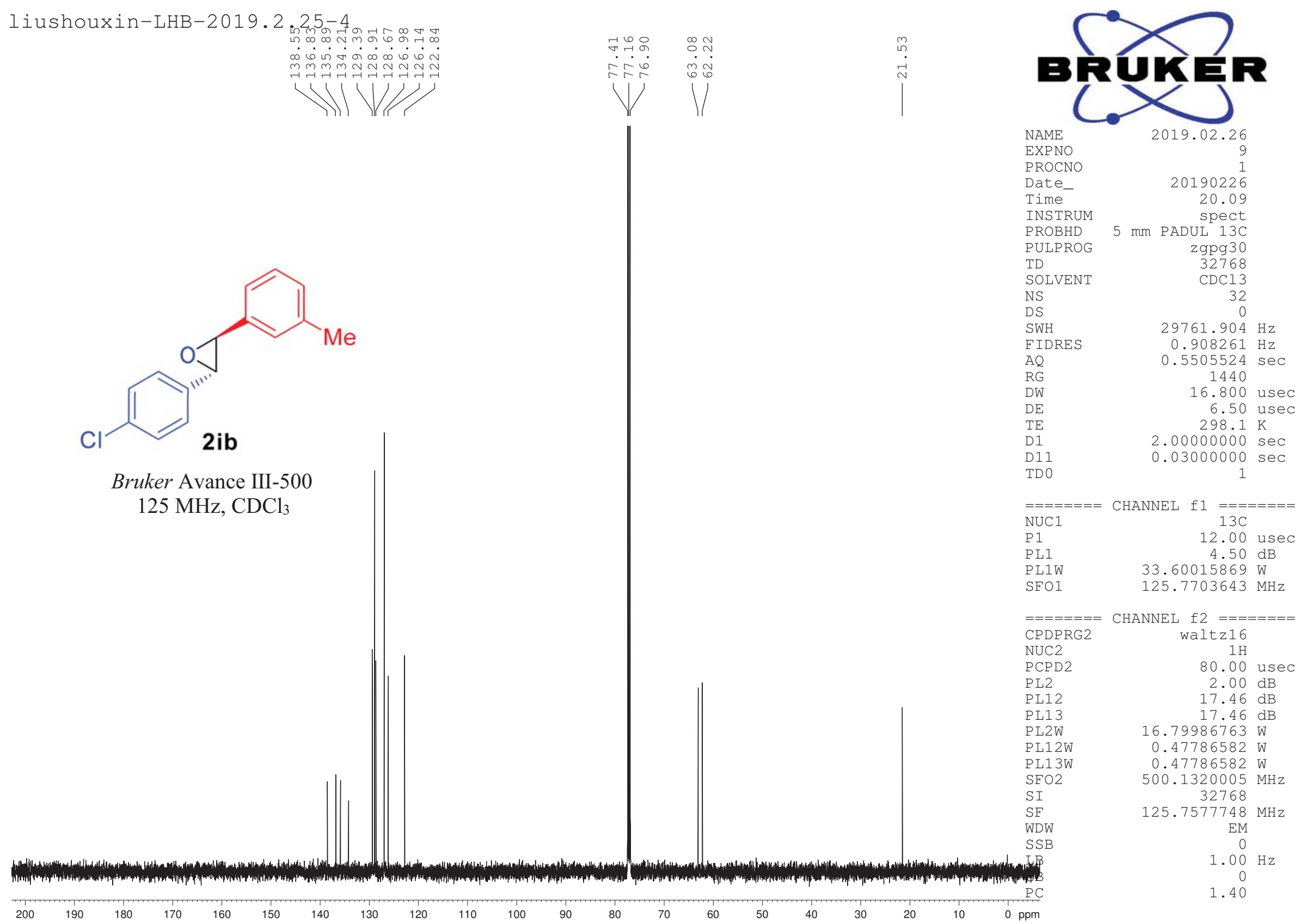




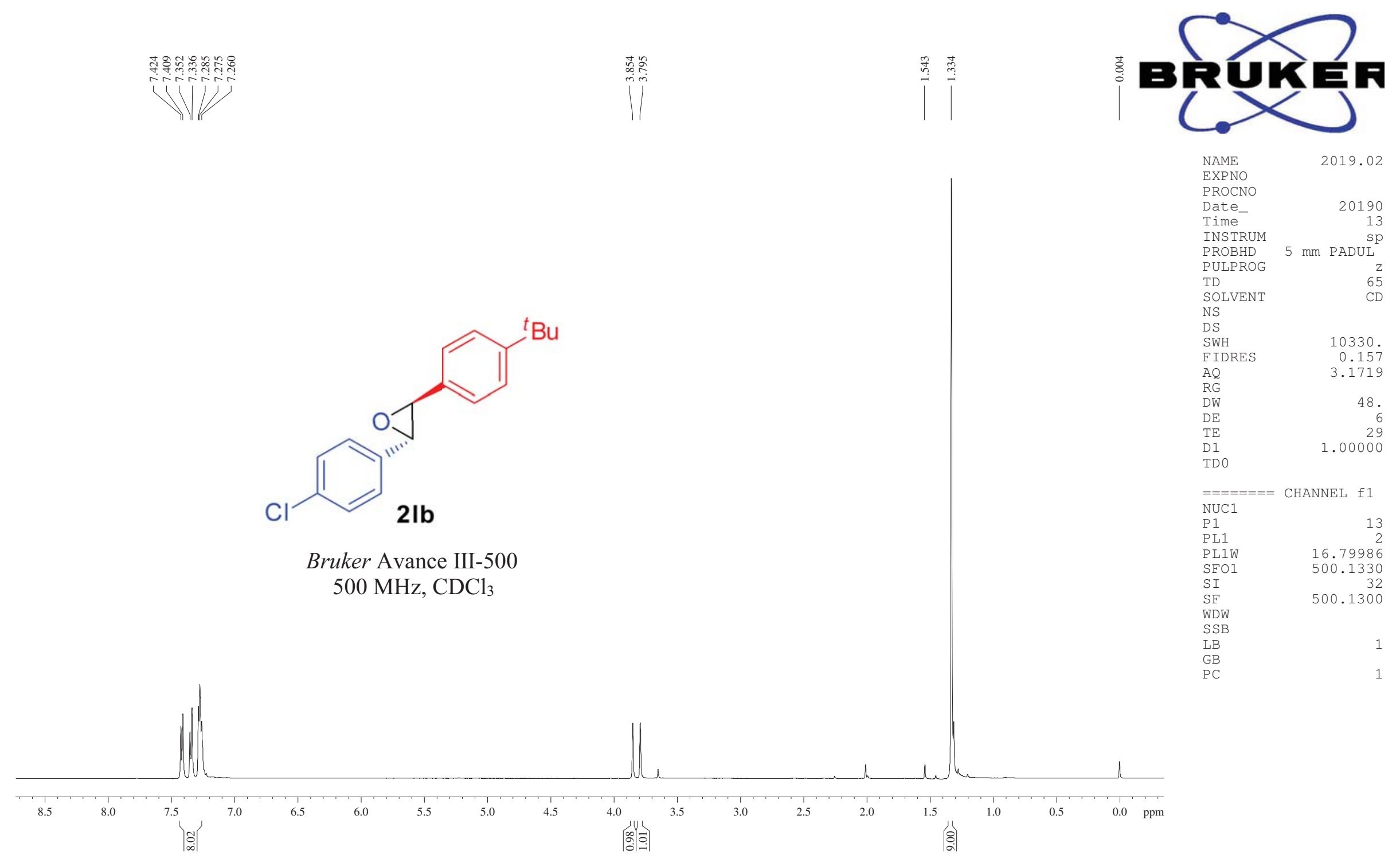




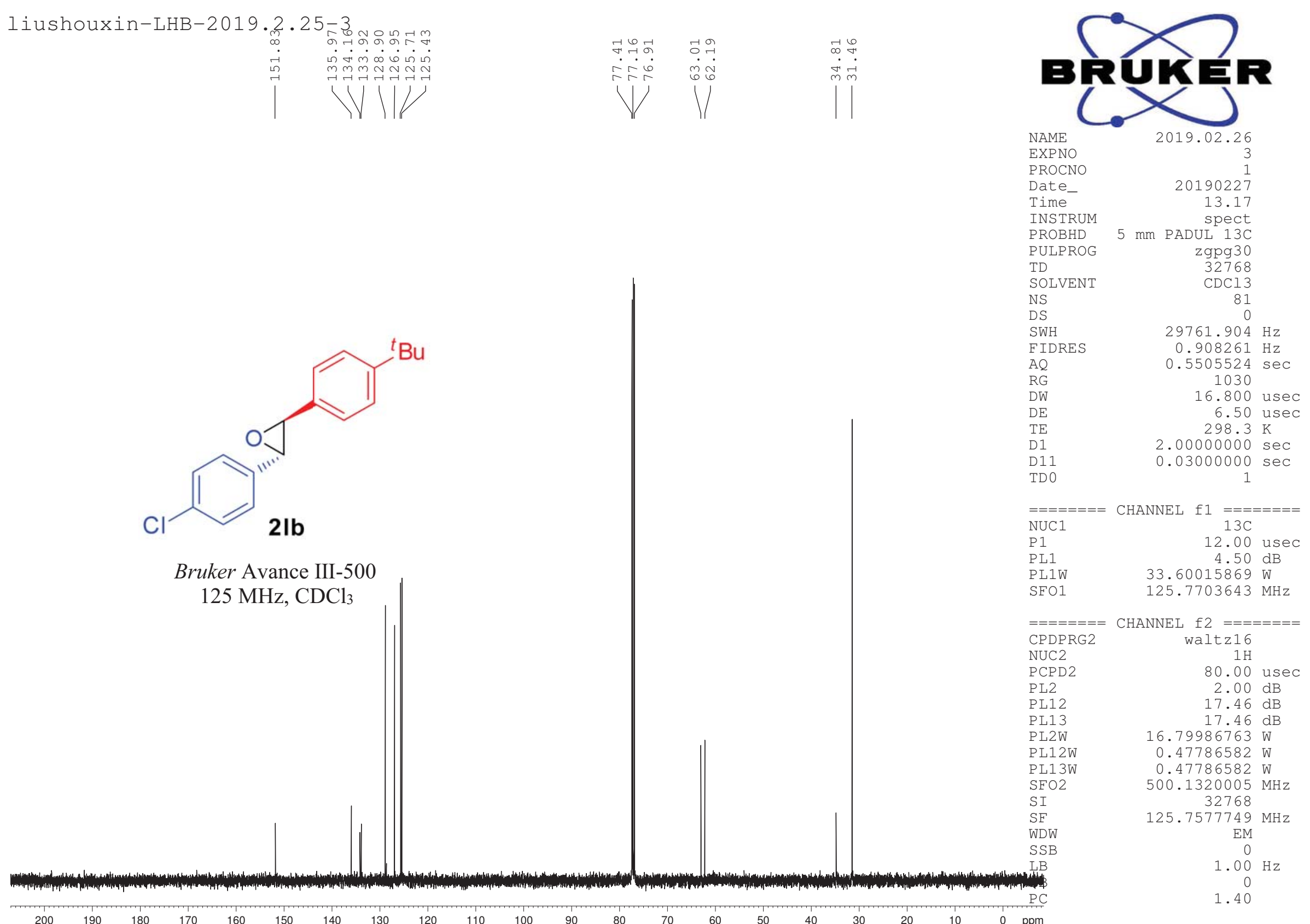




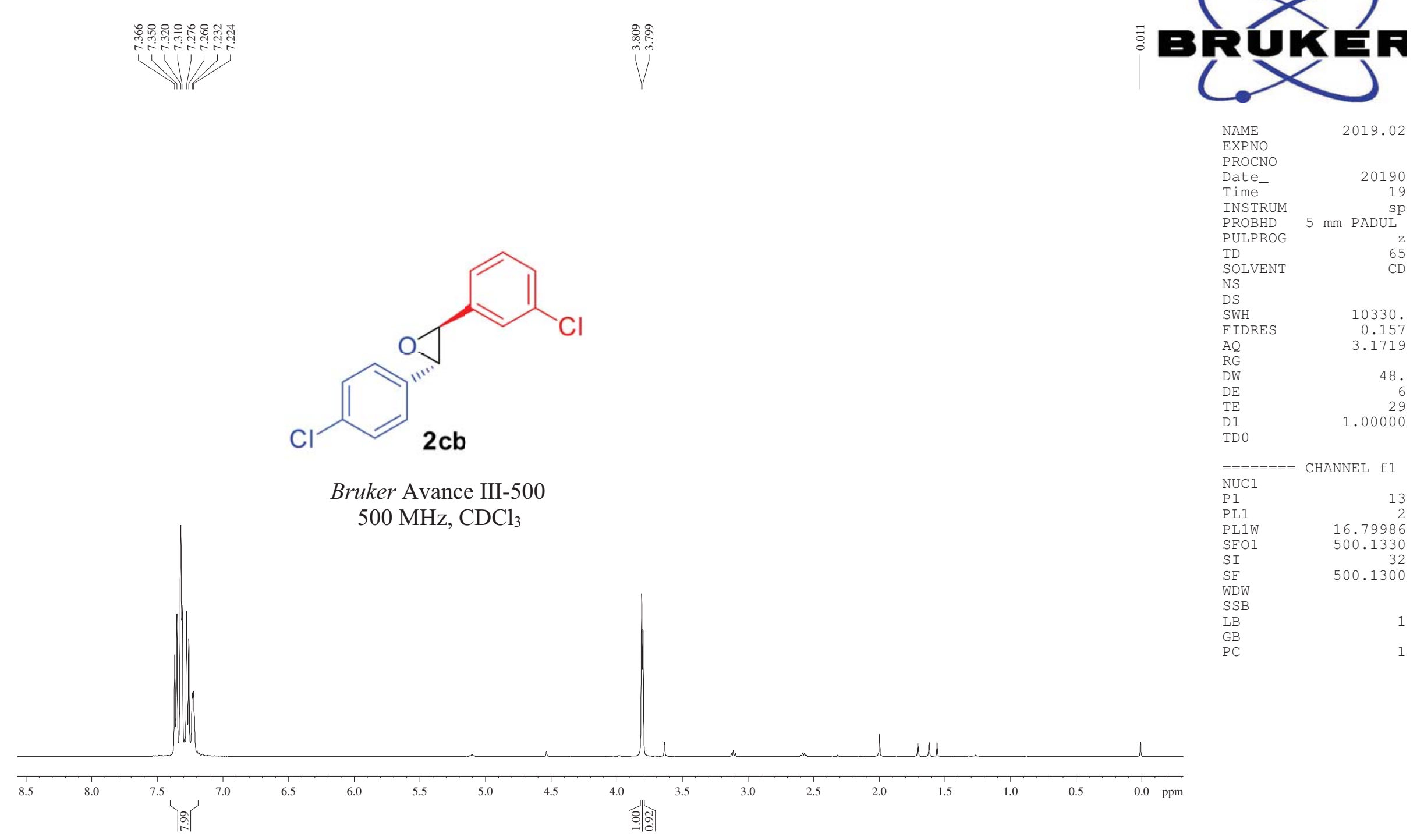




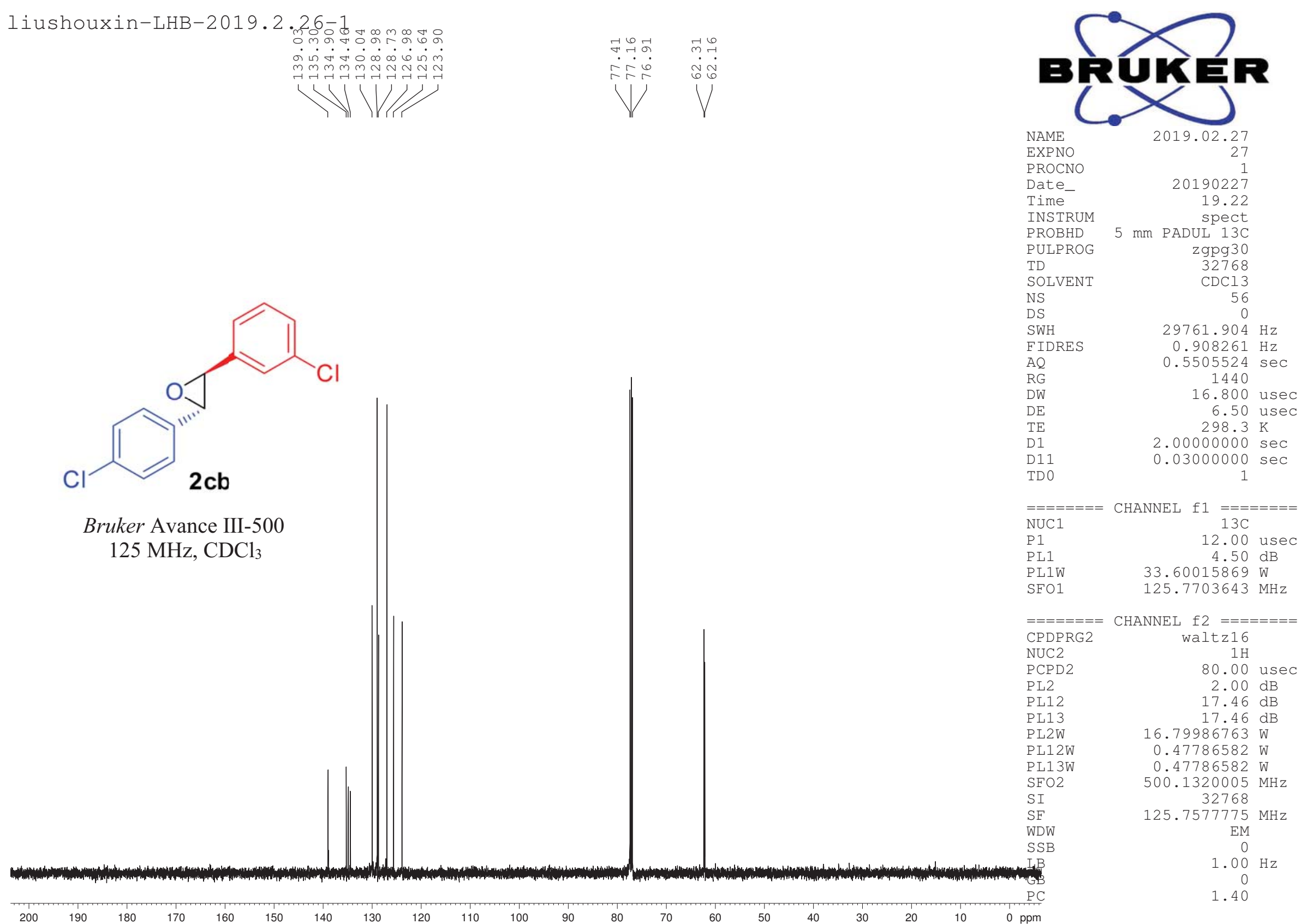




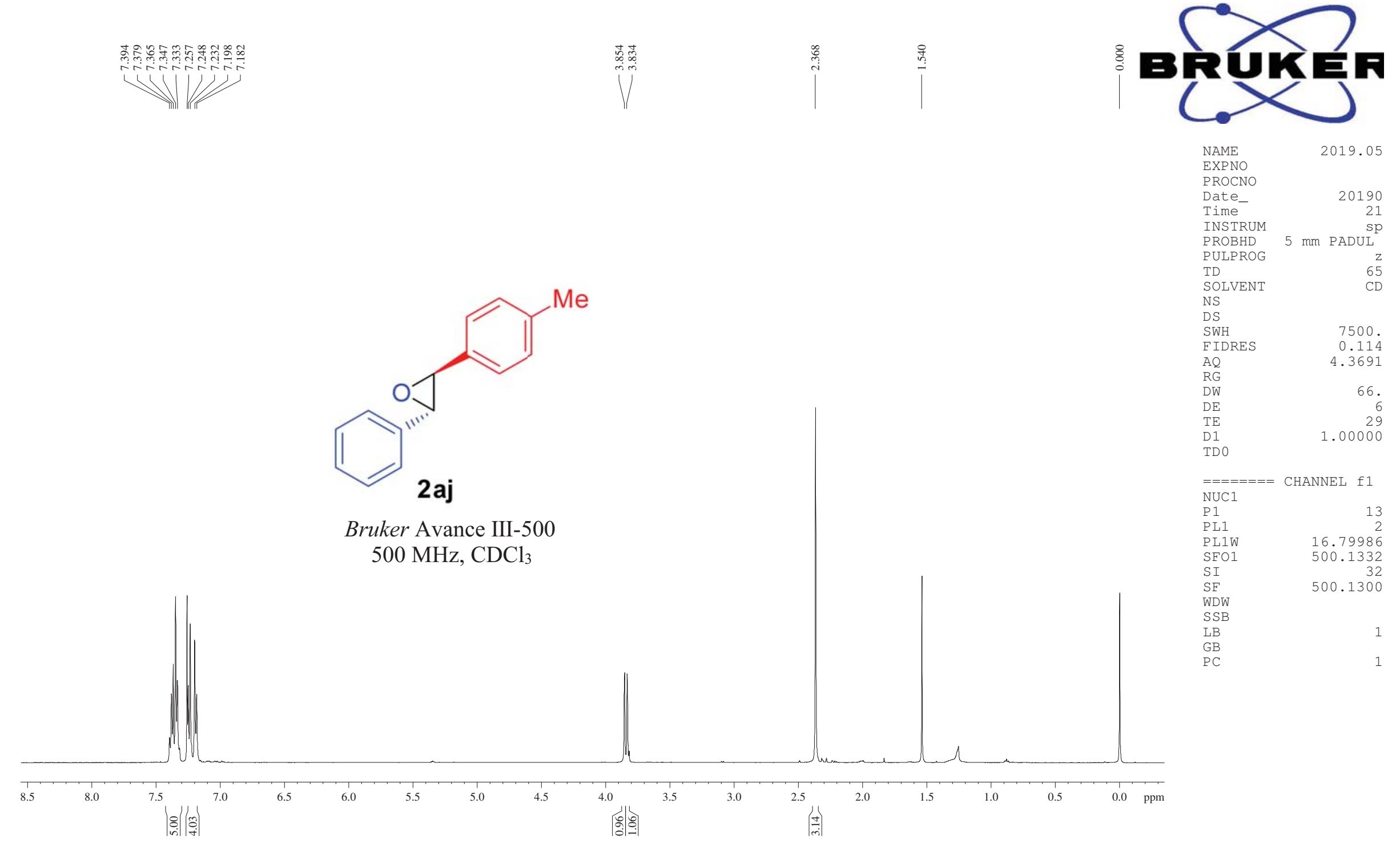




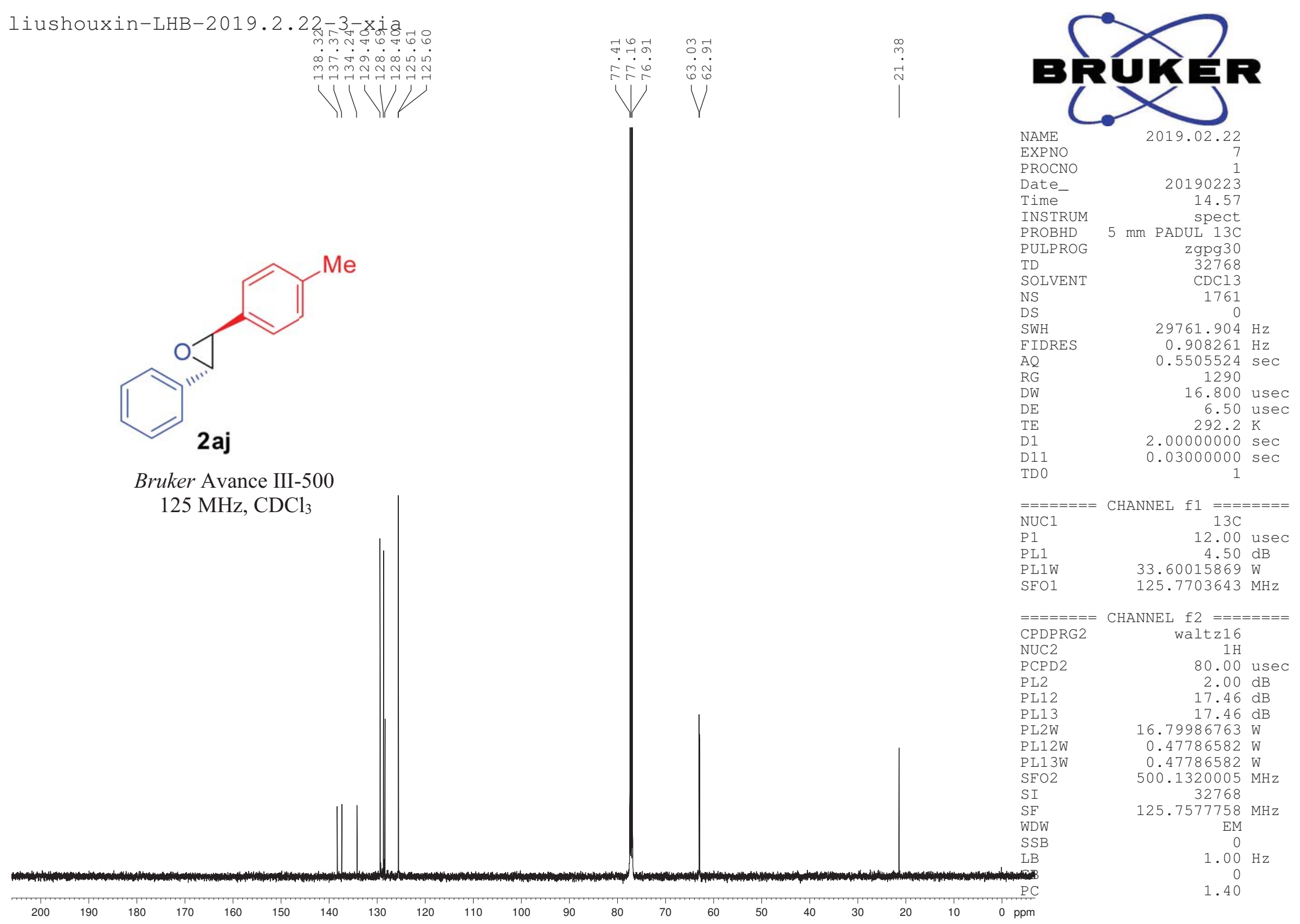




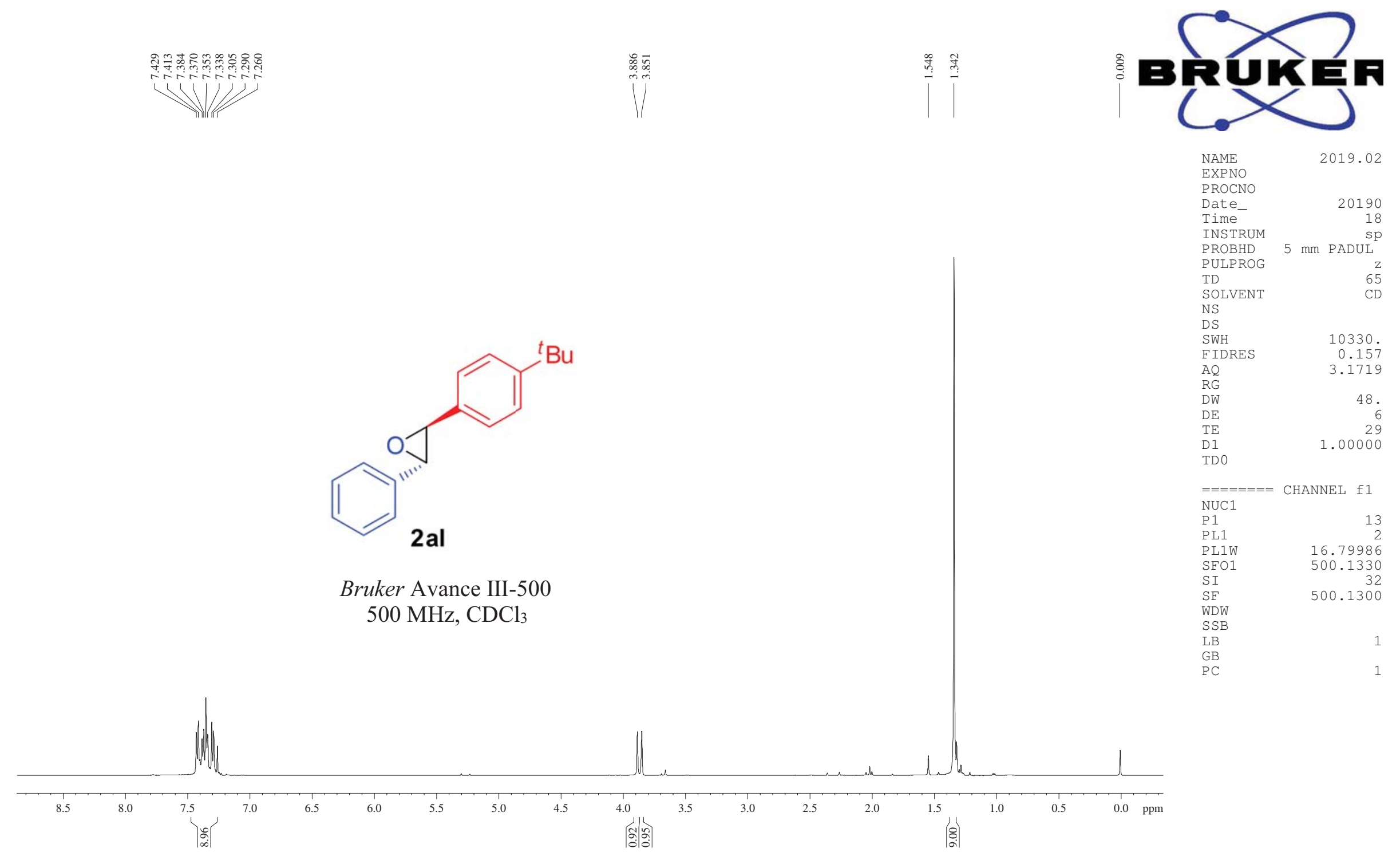




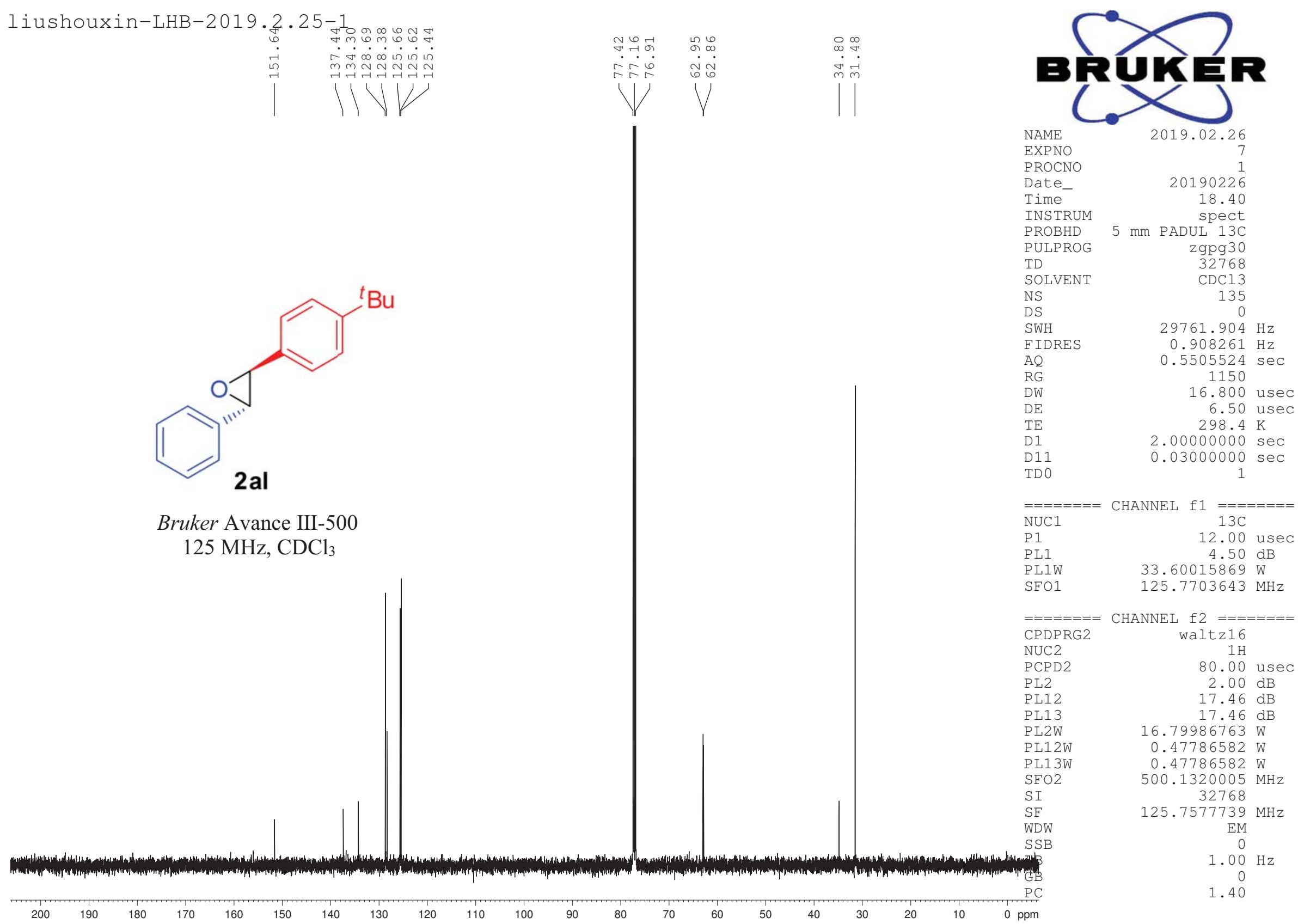




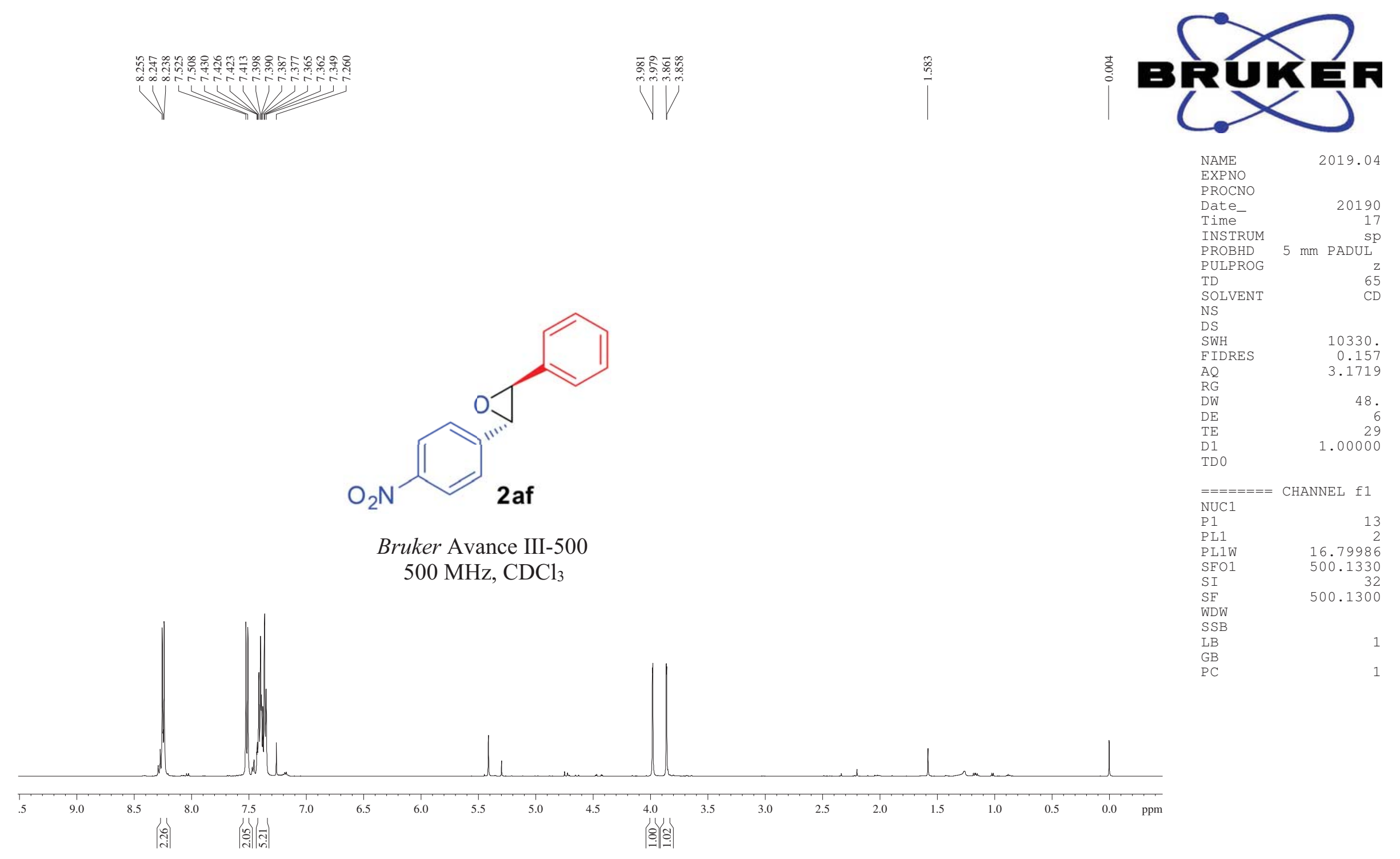




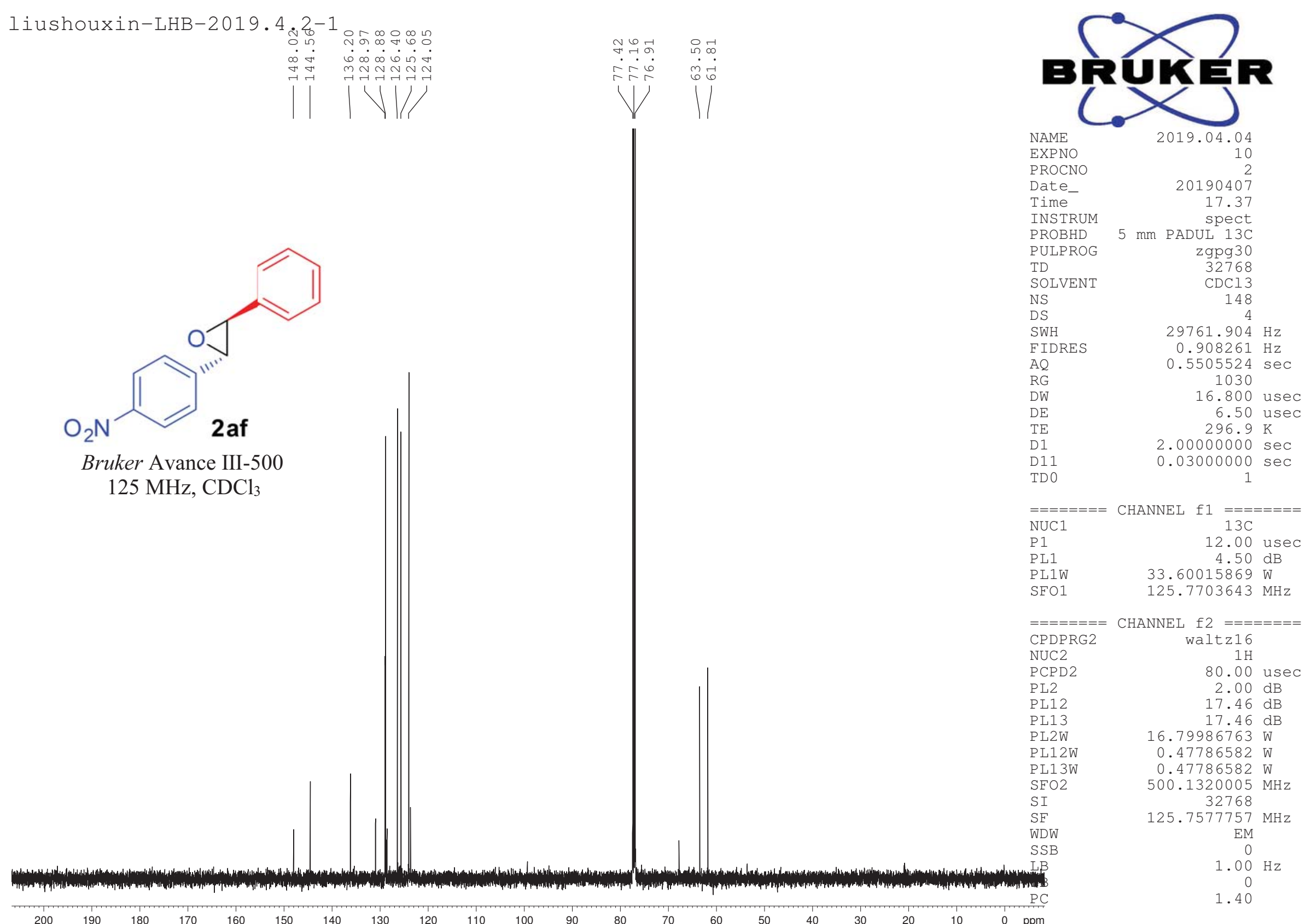




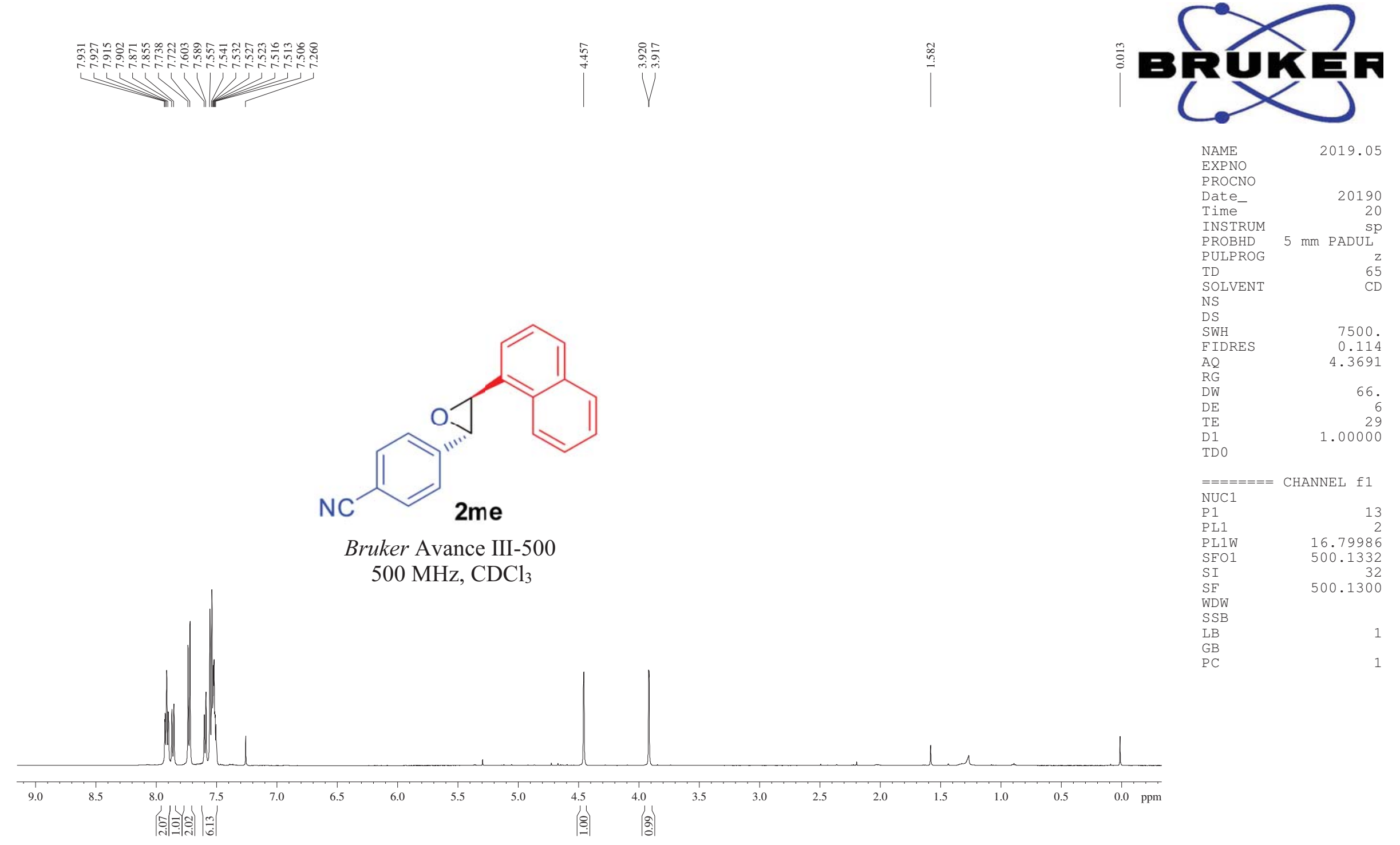




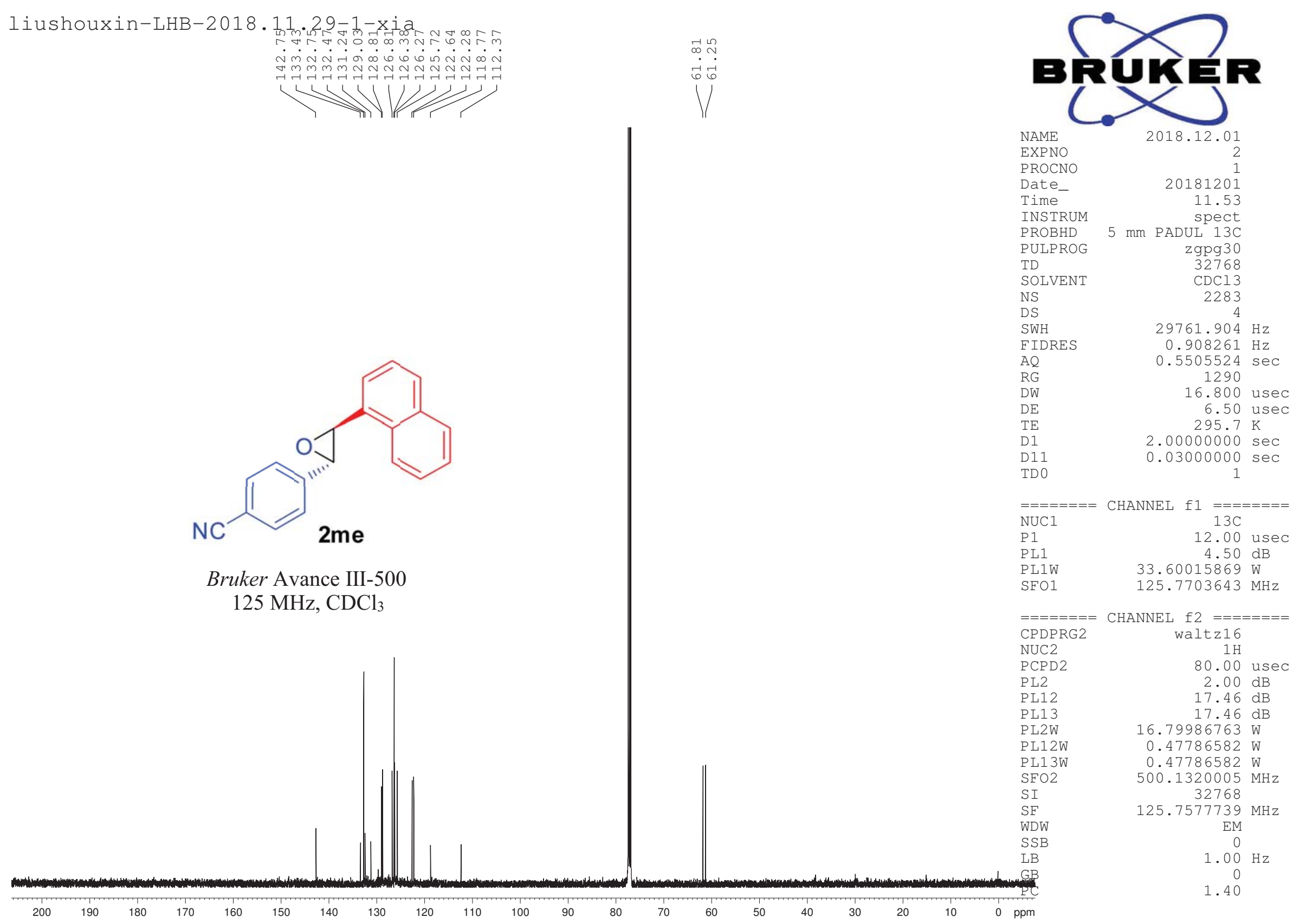




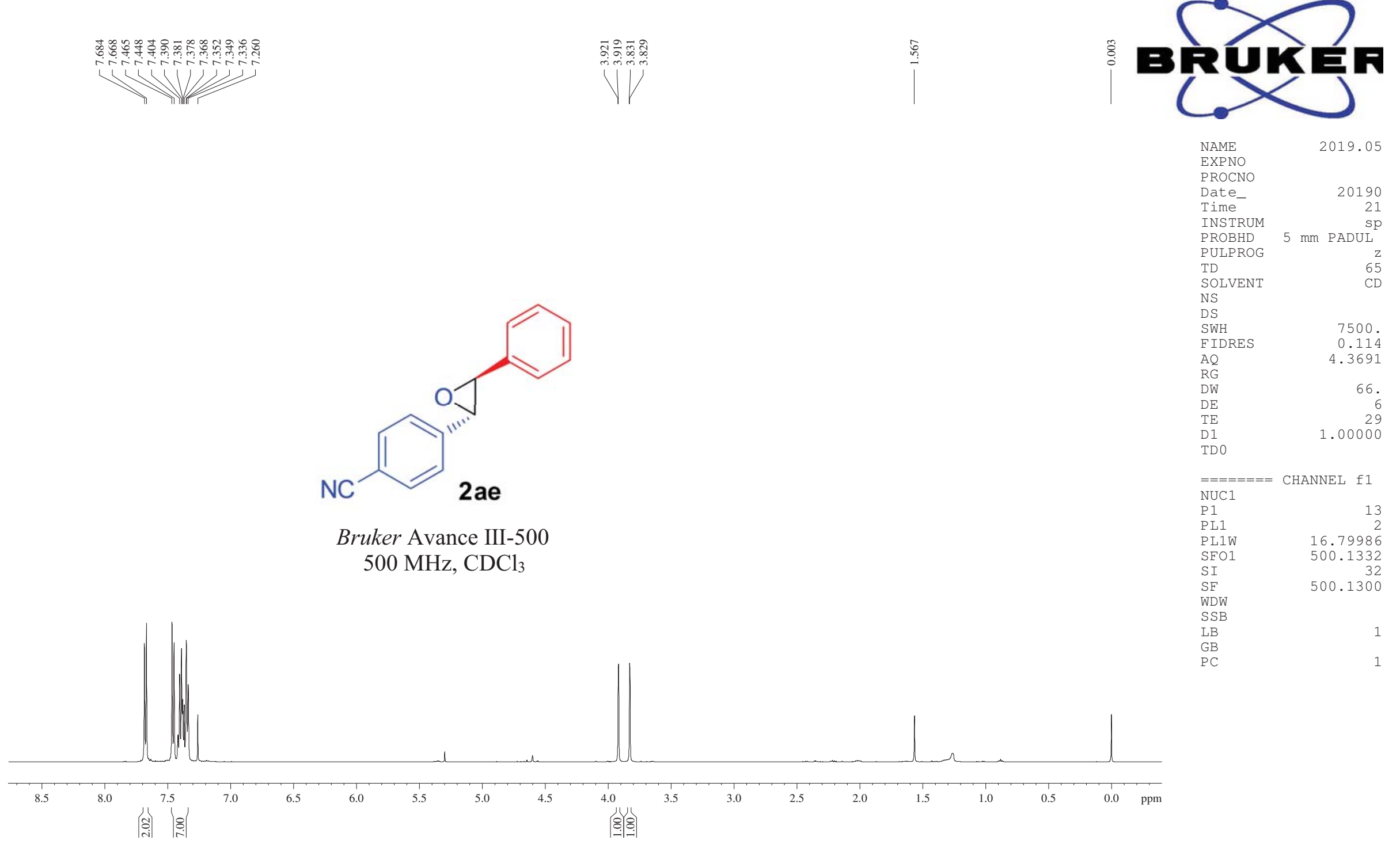




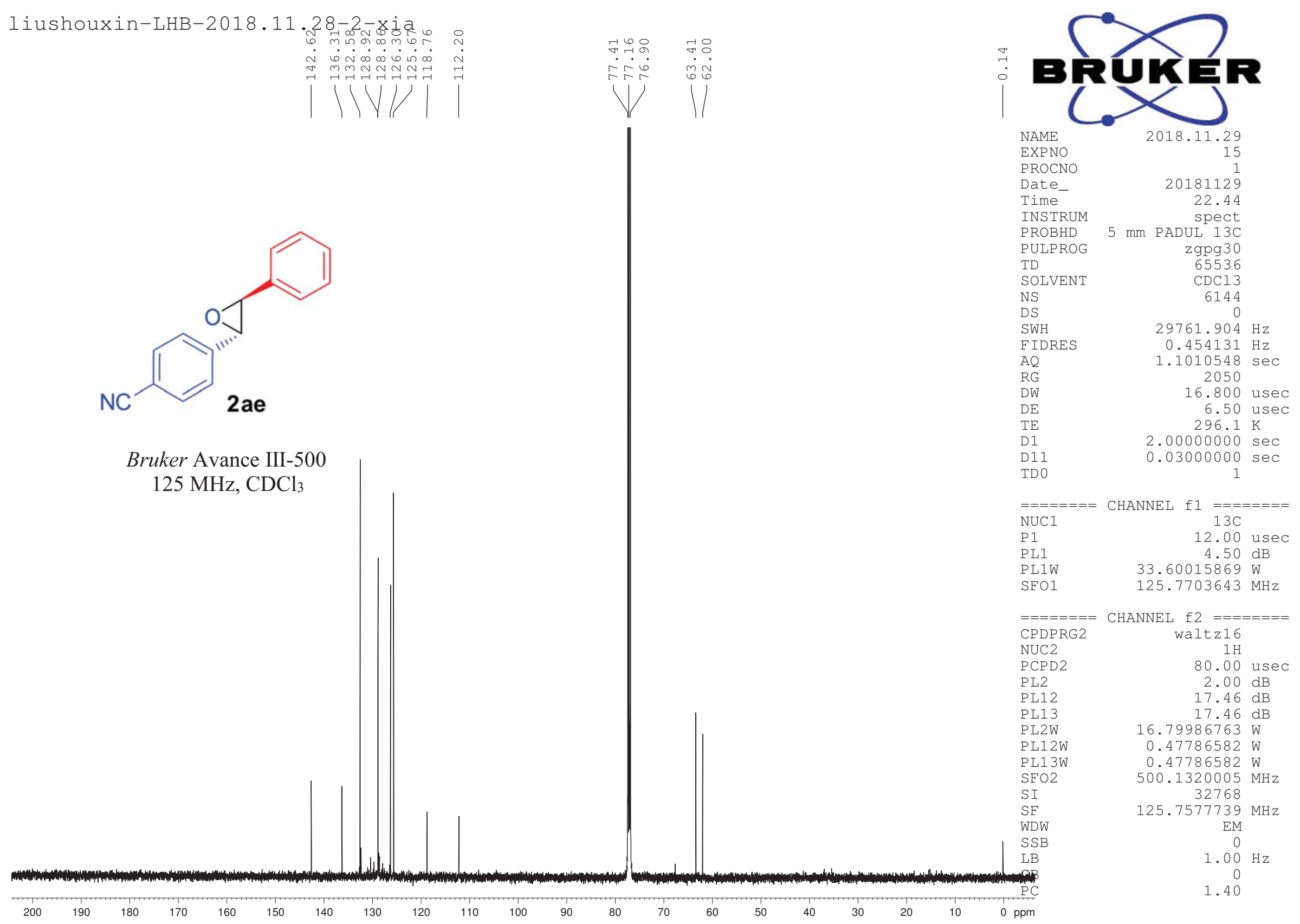




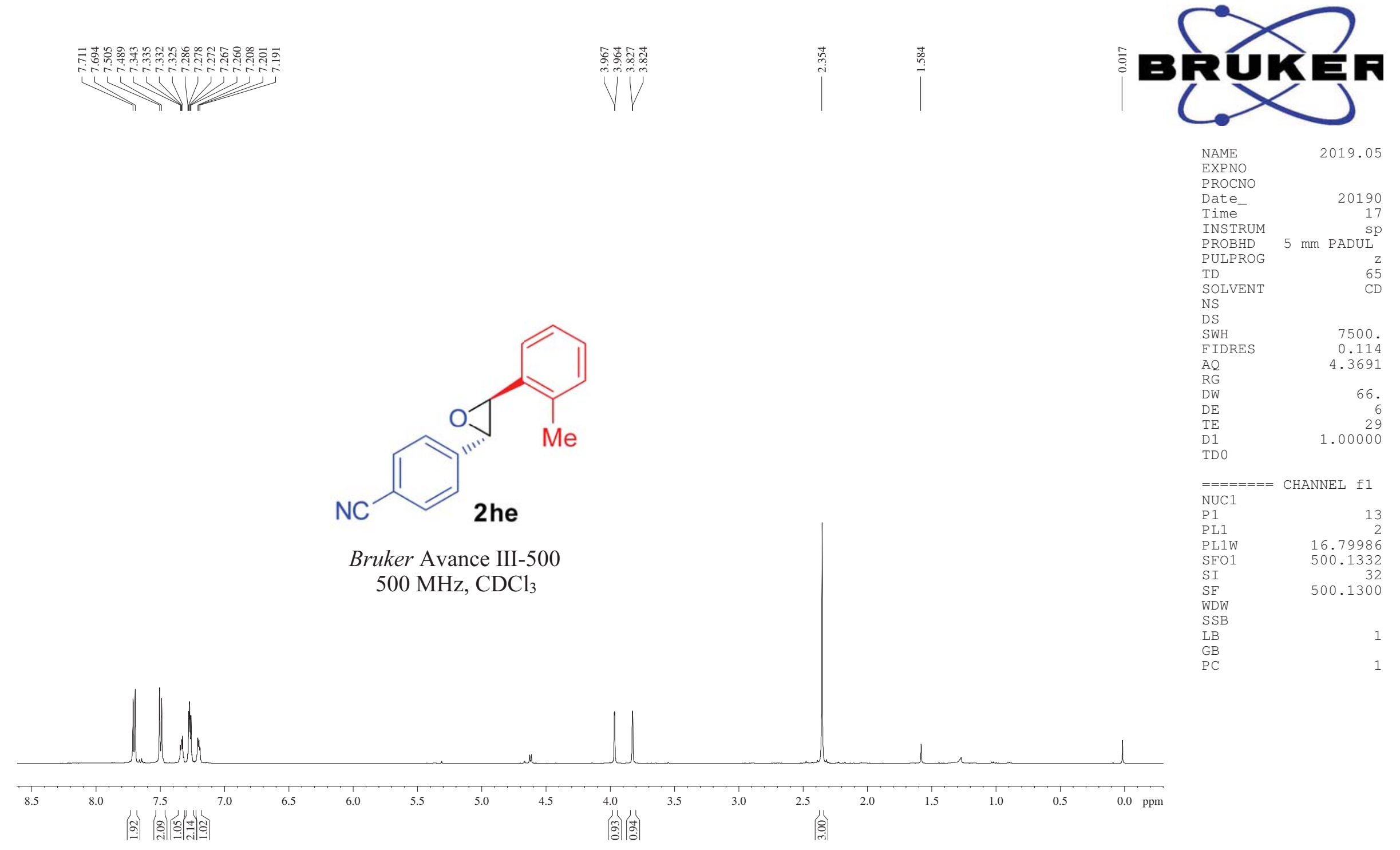




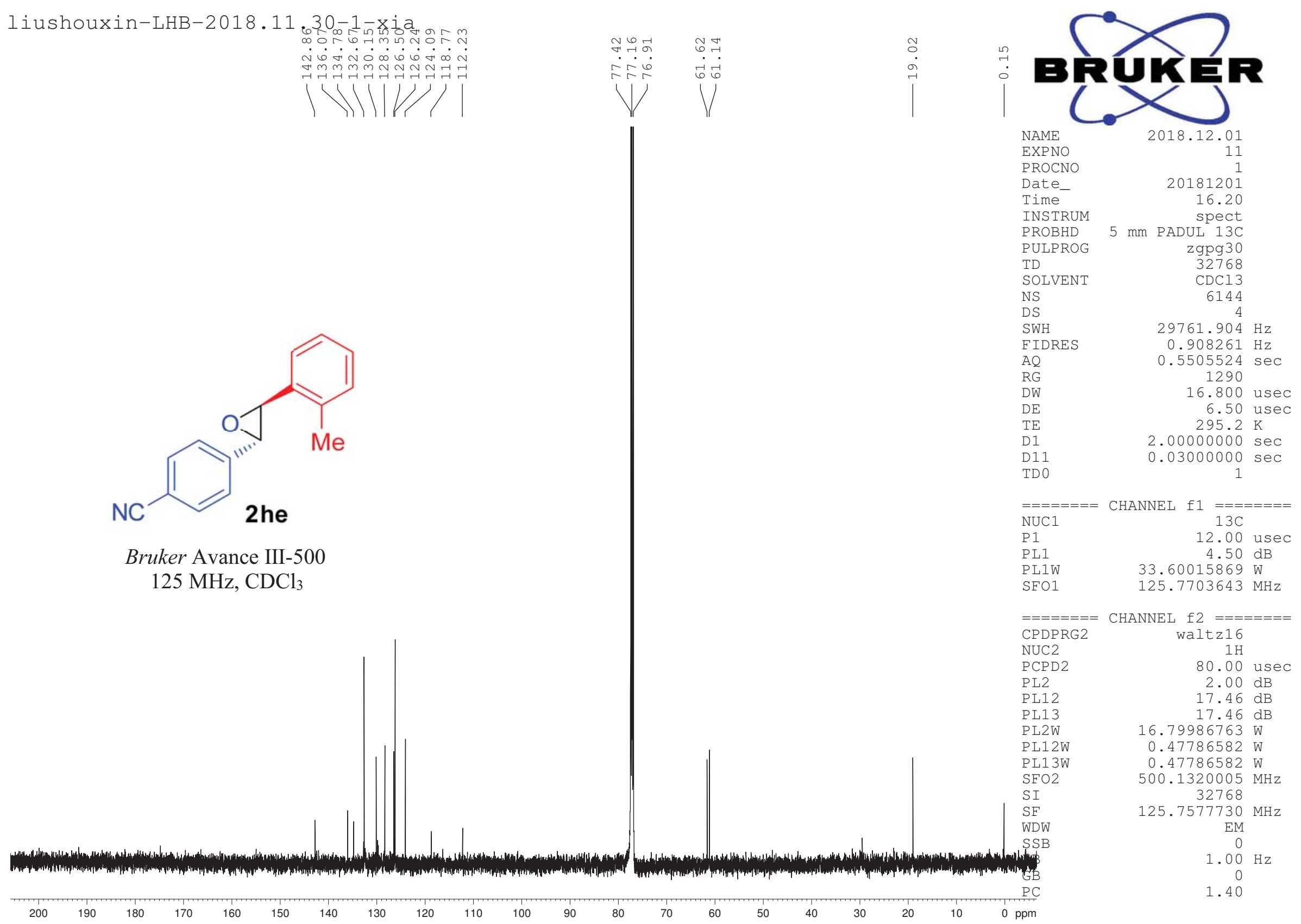




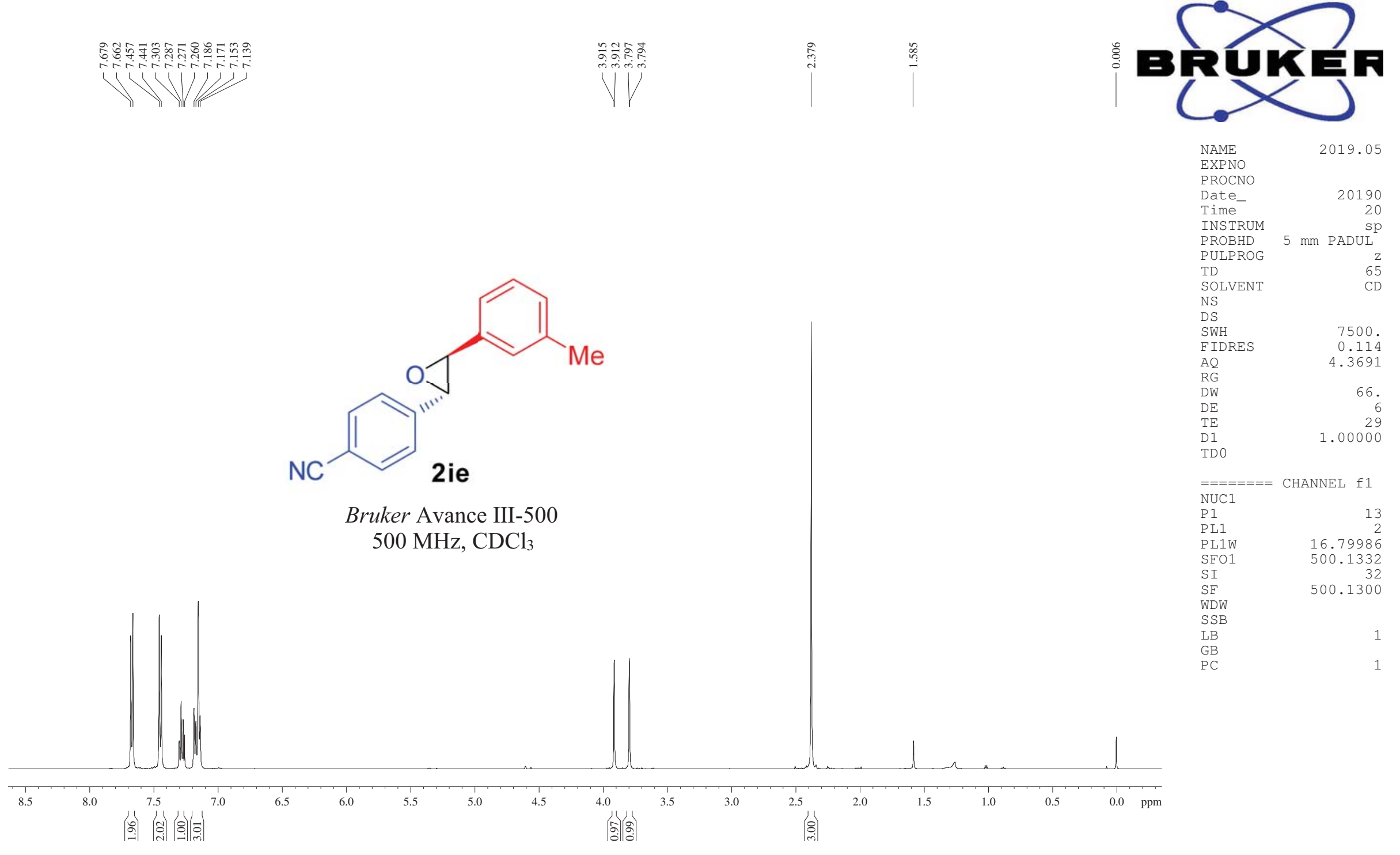




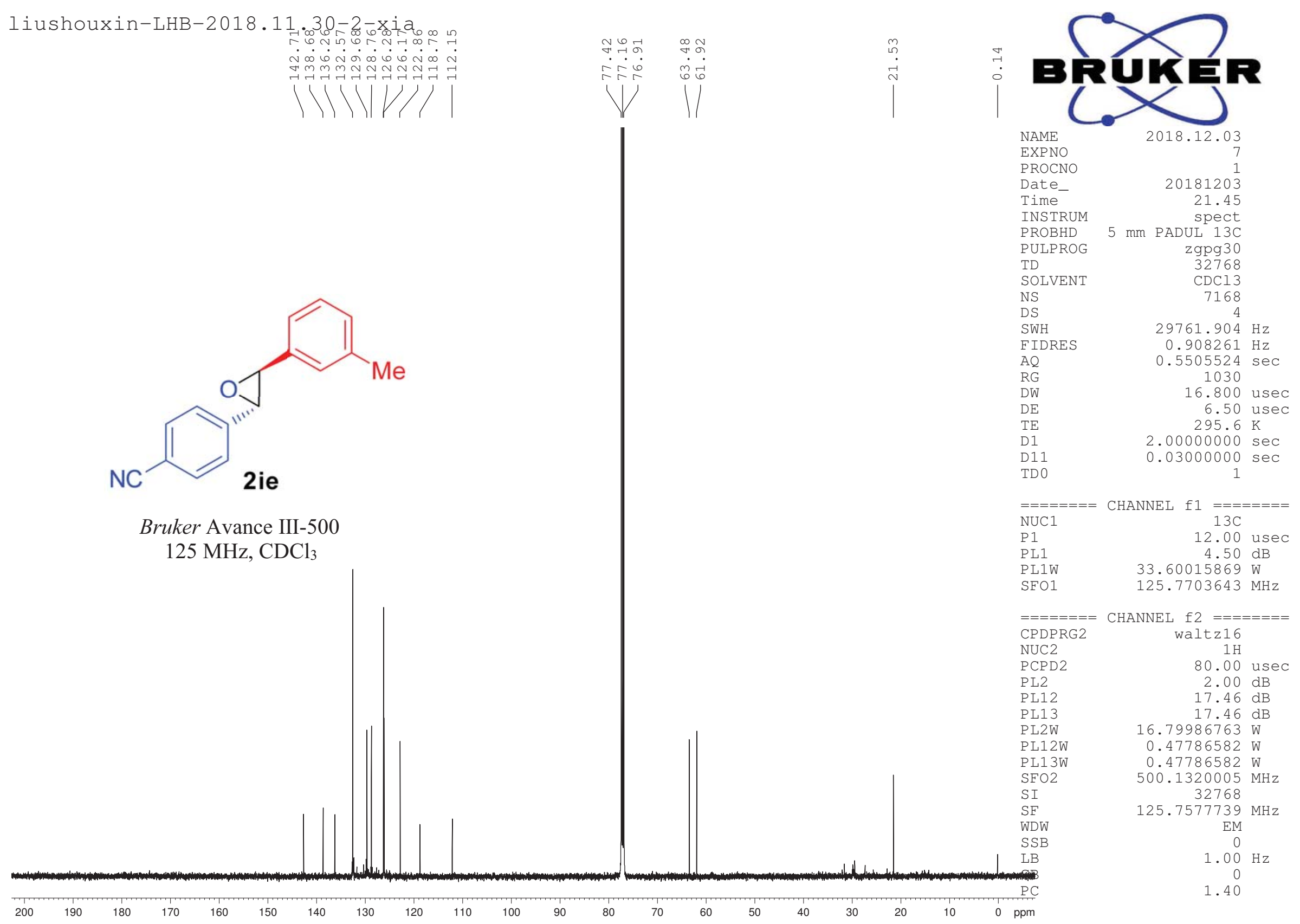




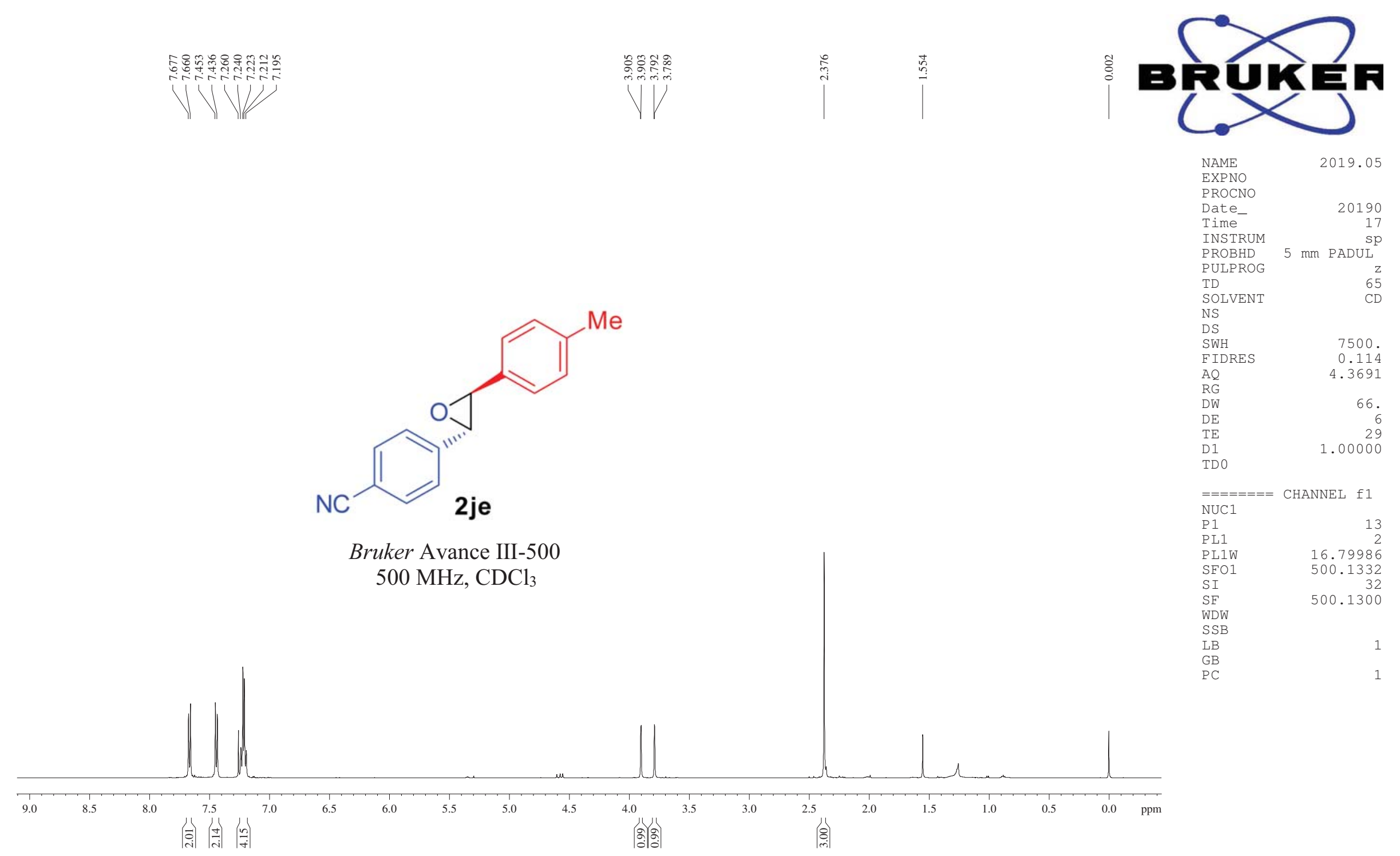




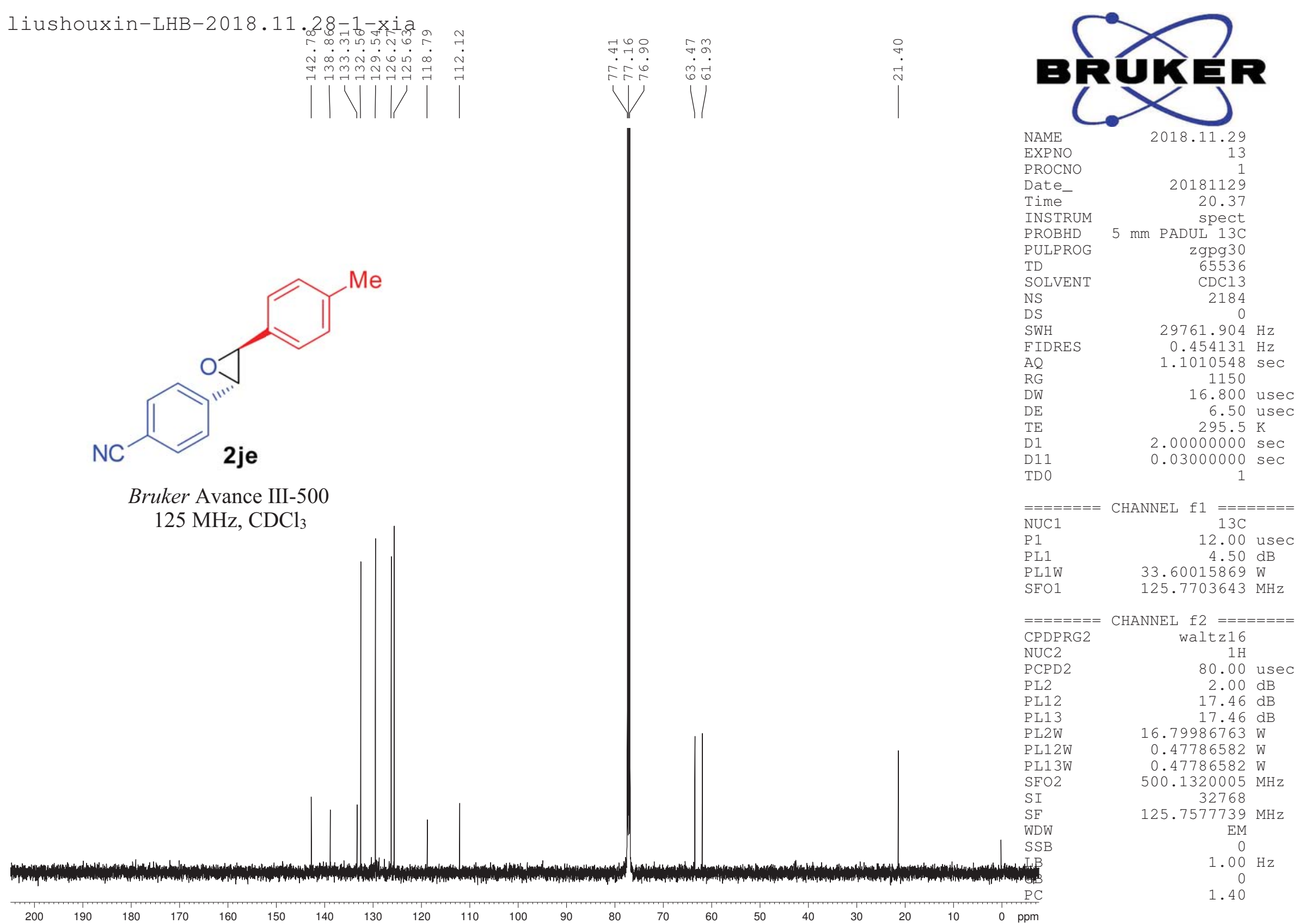




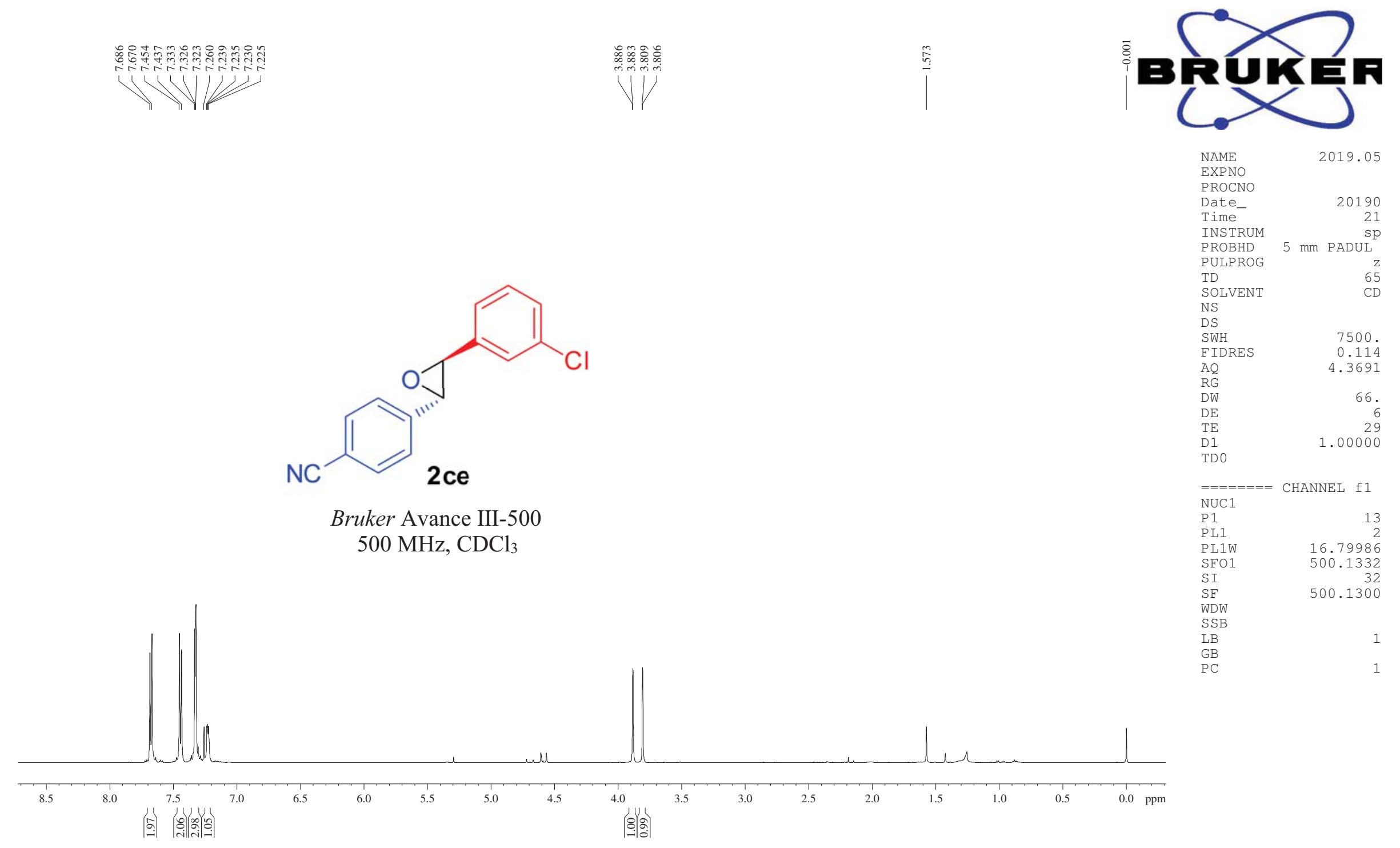




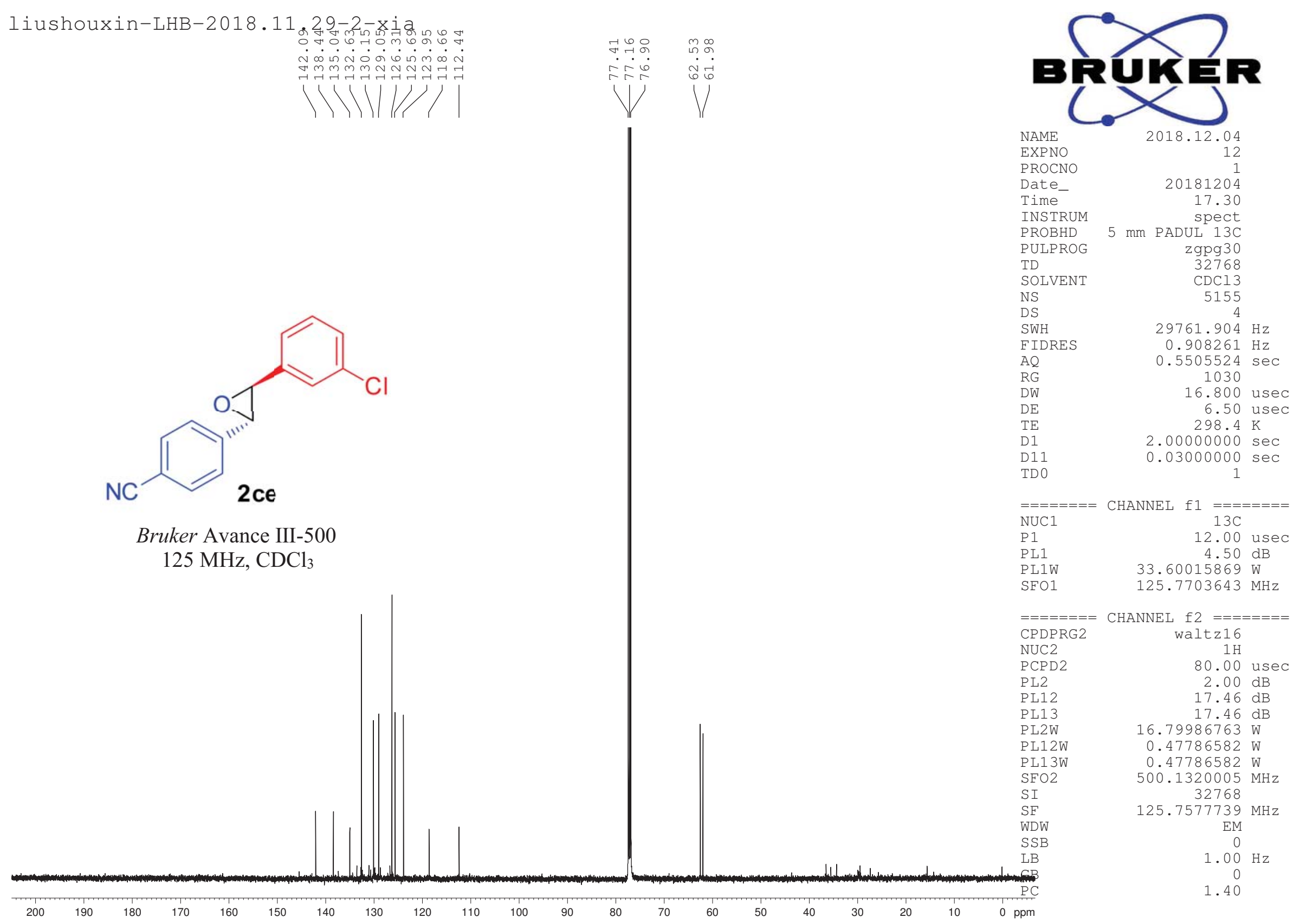




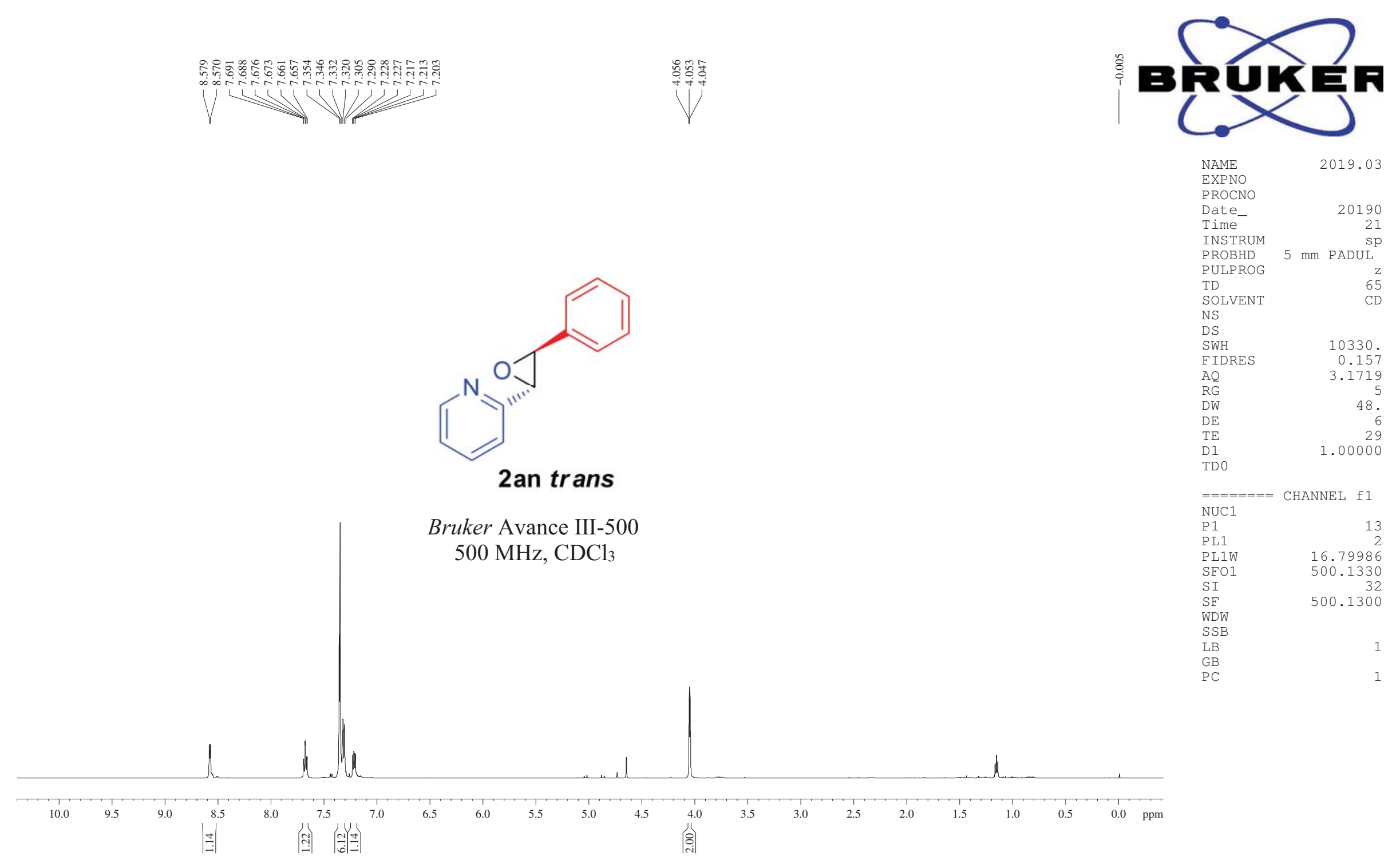




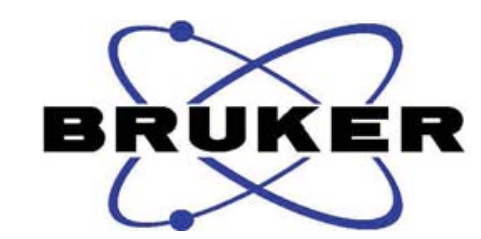

NAME

EXPNO

PROCNO

Date_

INSTRUM

PROBHD

PULPROG

TD

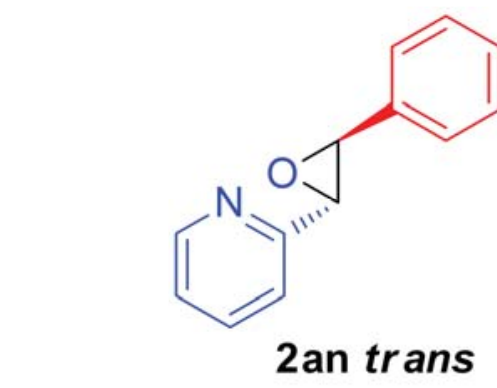

SOLVENT

DS

SWH

FIDRES

FID
AQ
RG
DW

DW

TE

D 1
D11

TD 0

2019.03 .28

20190328

21.09

spect

$5 \mathrm{~mm}$ PADUL 13C

zgpg 30
65536

$\mathrm{CDCl} 3$

23
4

$29761.904 \mathrm{~Hz}$

$0.454131 \mathrm{~Hz}$

$1.1010548 \mathrm{sec}$

$$
1030 \mathrm{sec}
$$

16.800 usec

$6.50 \mathrm{usec}$
$298.5 \mathrm{~K}$

$.00000000 \mathrm{sec}$

$0.03000000 \mathrm{sec}$

$=======$ CHANNEL

NUC1

PL1

PL1W
SFO1

$======$

$125 \mathrm{MHz}, \mathrm{CDCl}_{3}$

$======$

CPDPRG

NUC2

PCPD2

PL2
PL12

PL12
PL13

PL13
PL2W

PL2W
PL12W

PL12W
PL13W

$\mathrm{SFO} 2$

SI

SF
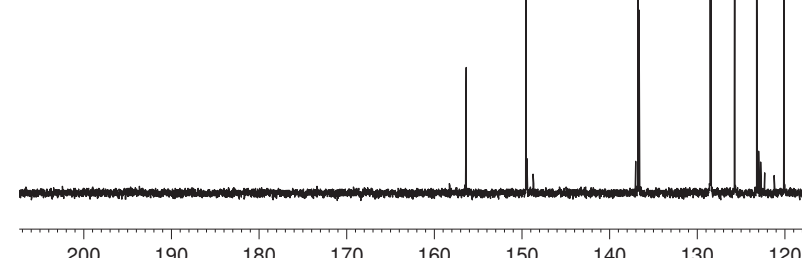

150

$140 \quad 130$

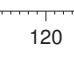

$20 \quad 110$

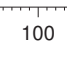

$100 \quad 90$
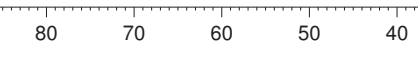


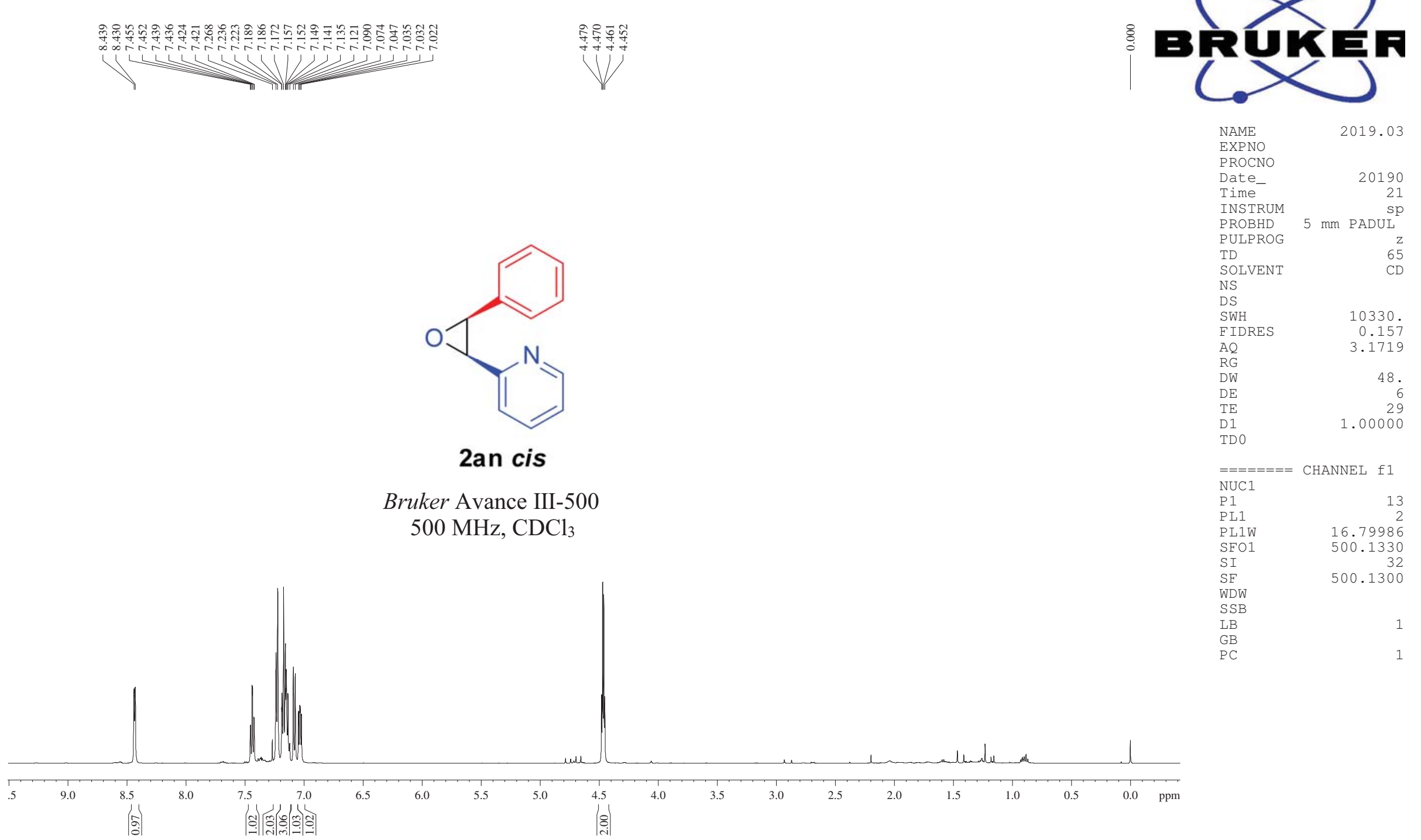




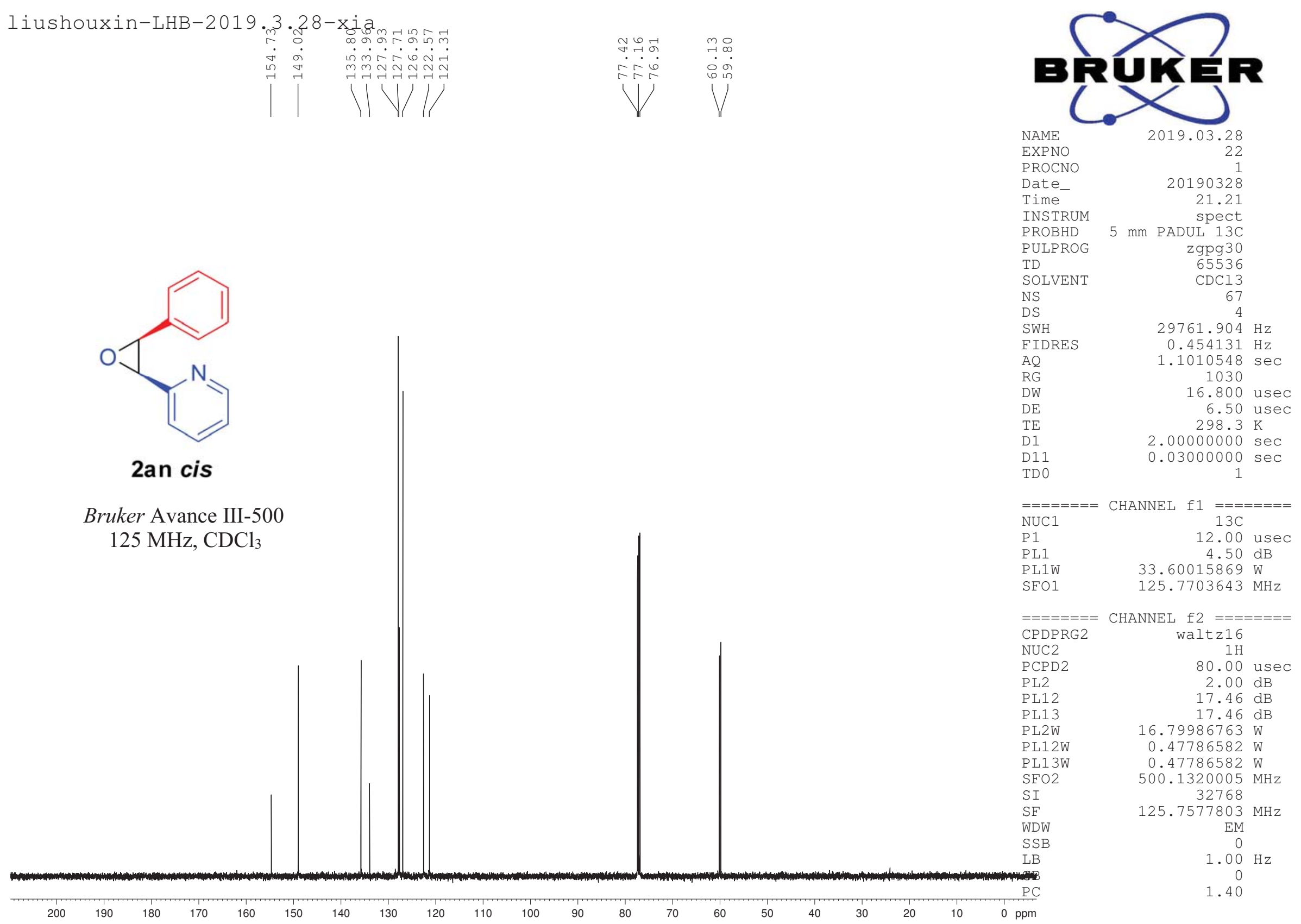




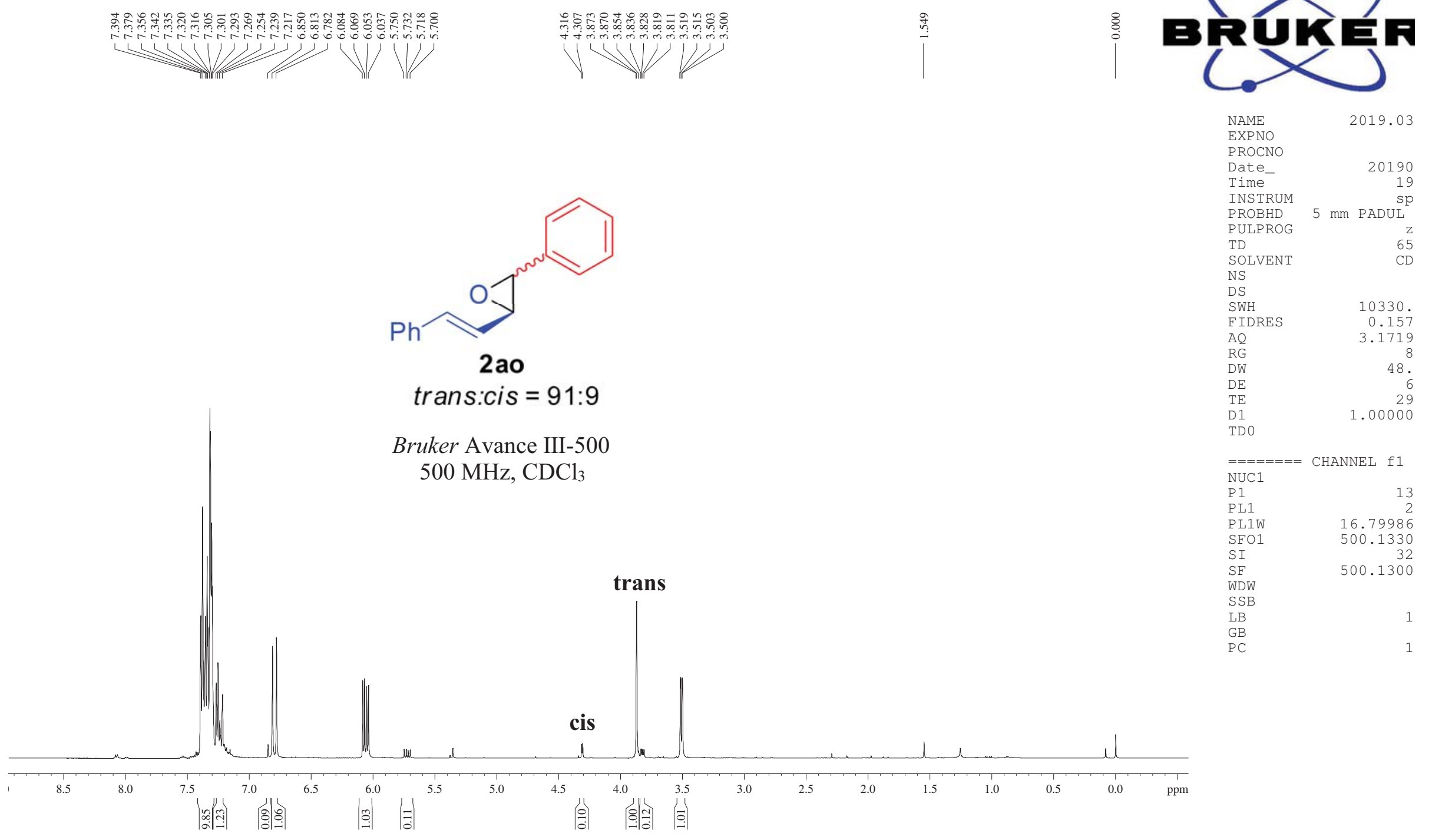



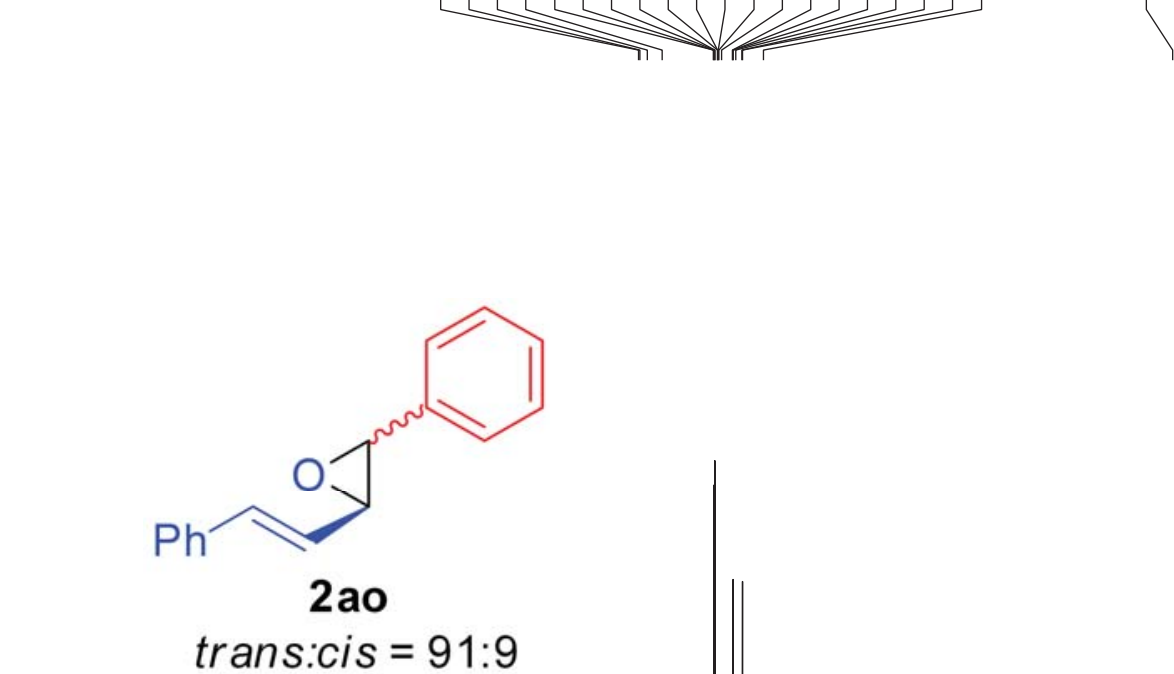

Bruker Avance III-500

$125 \mathrm{MHz}, \mathrm{CDCl}_{3}$

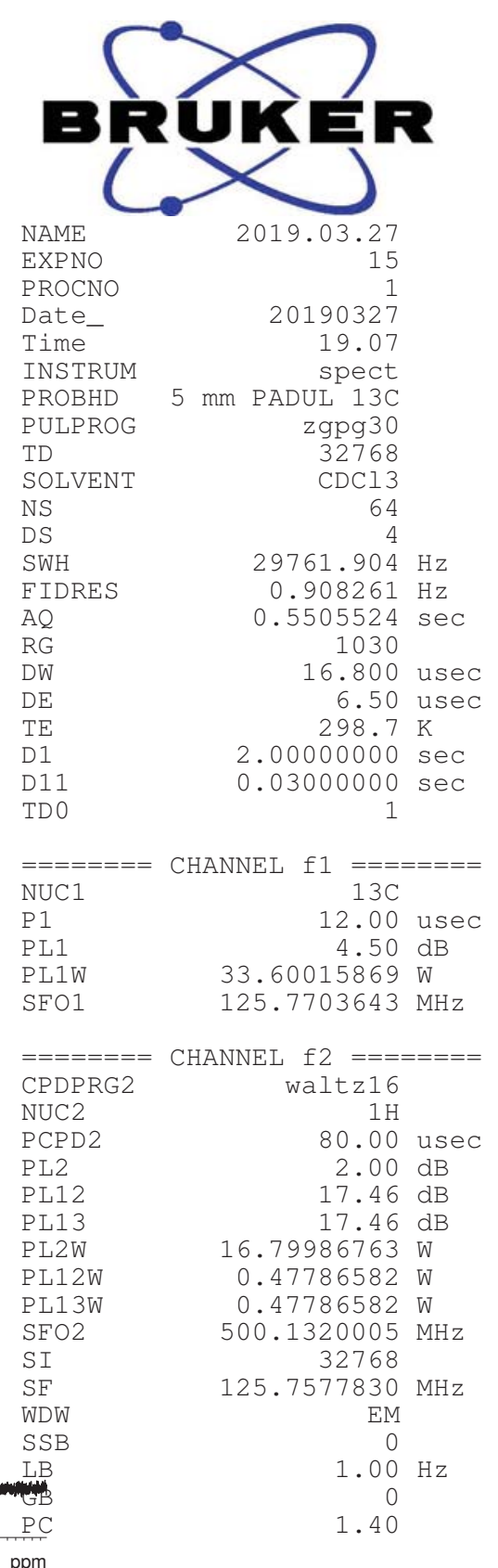




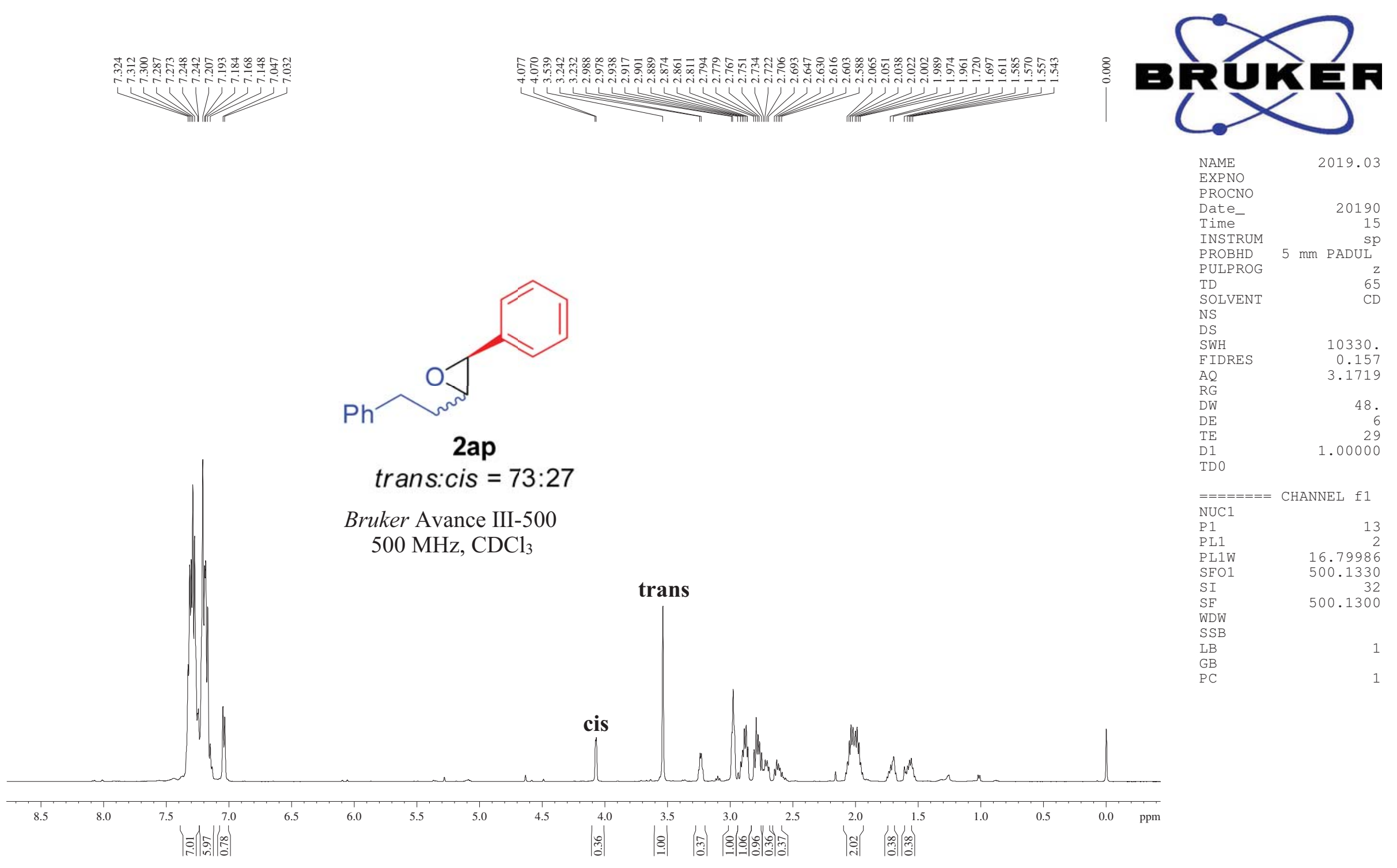




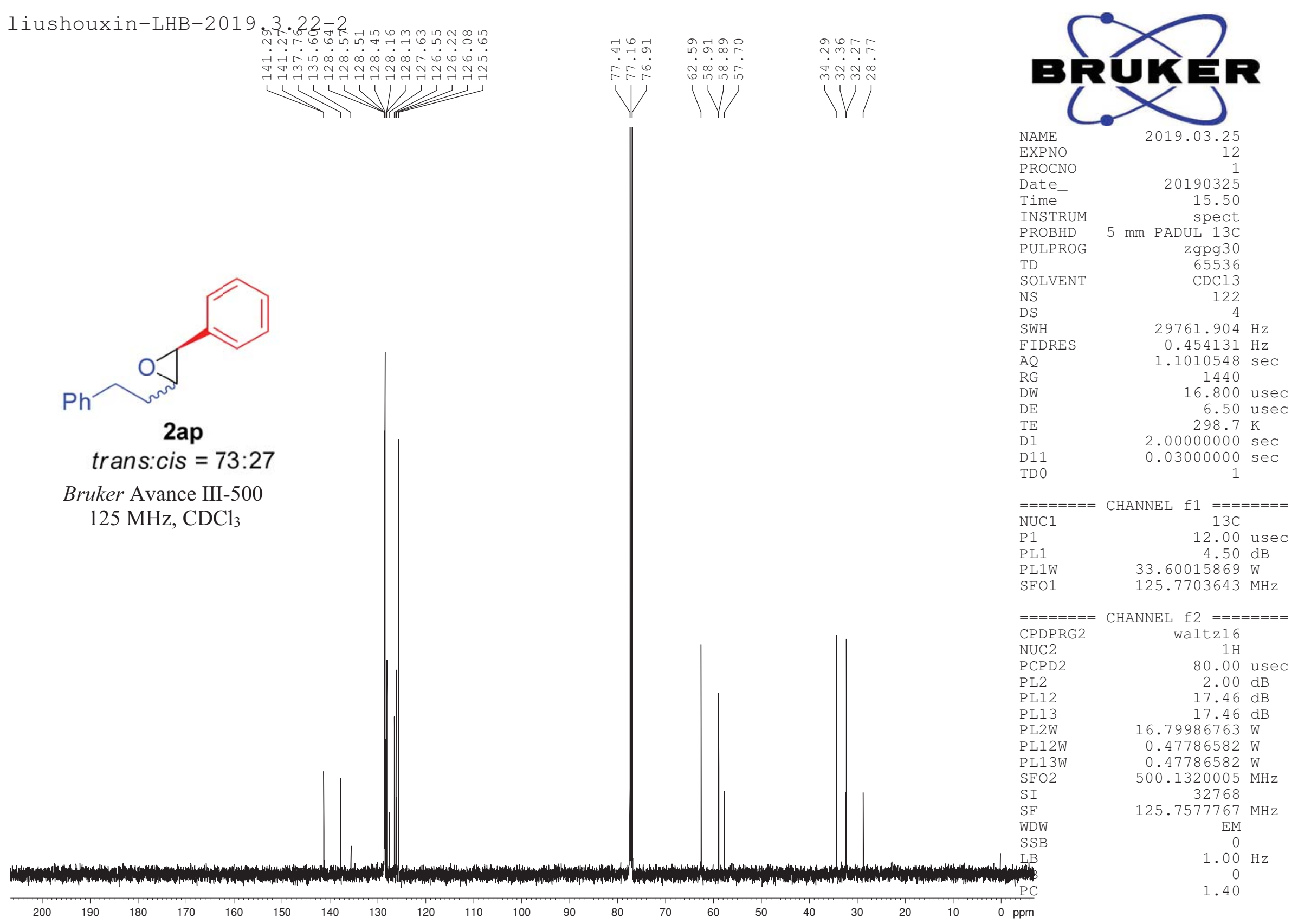




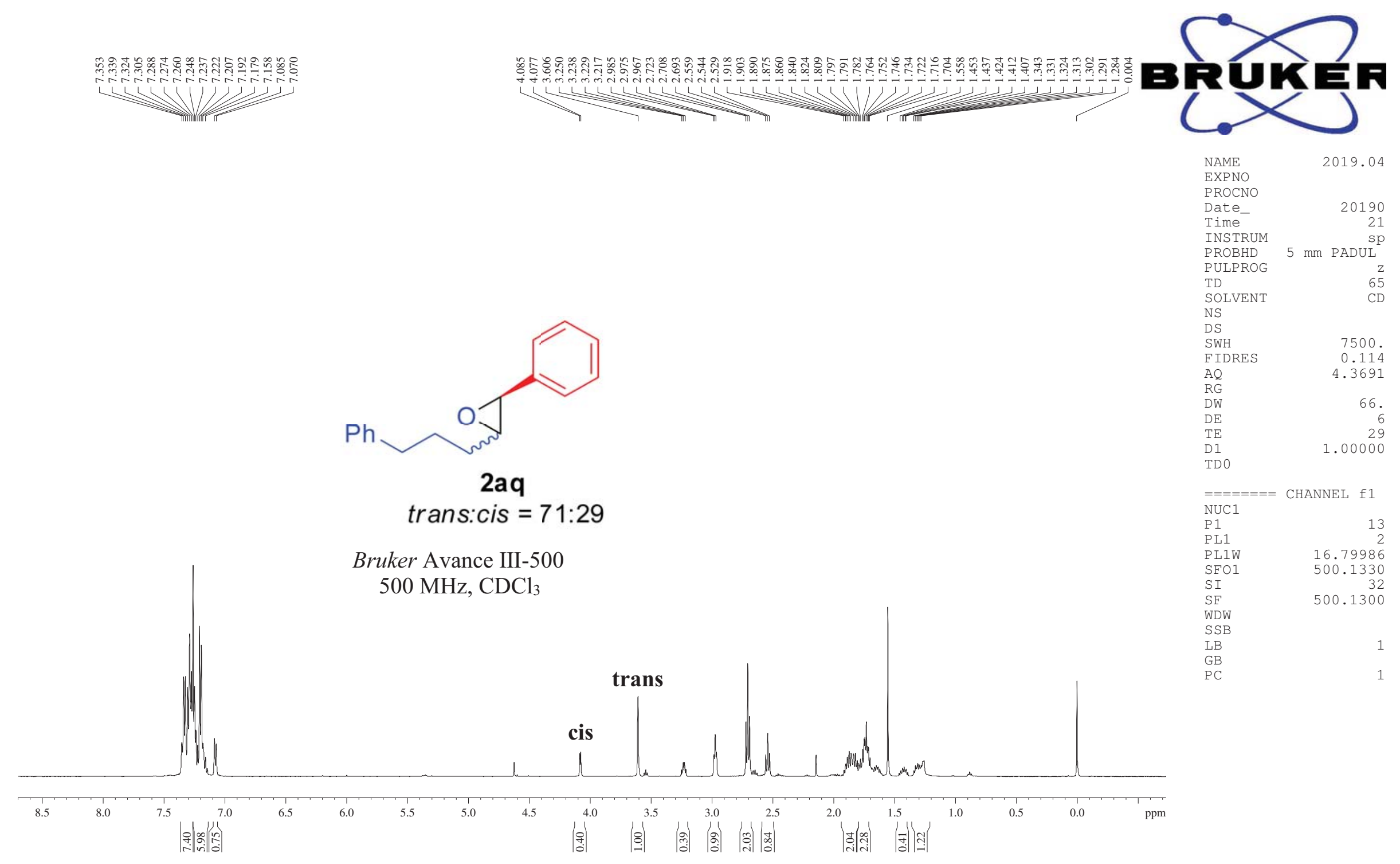




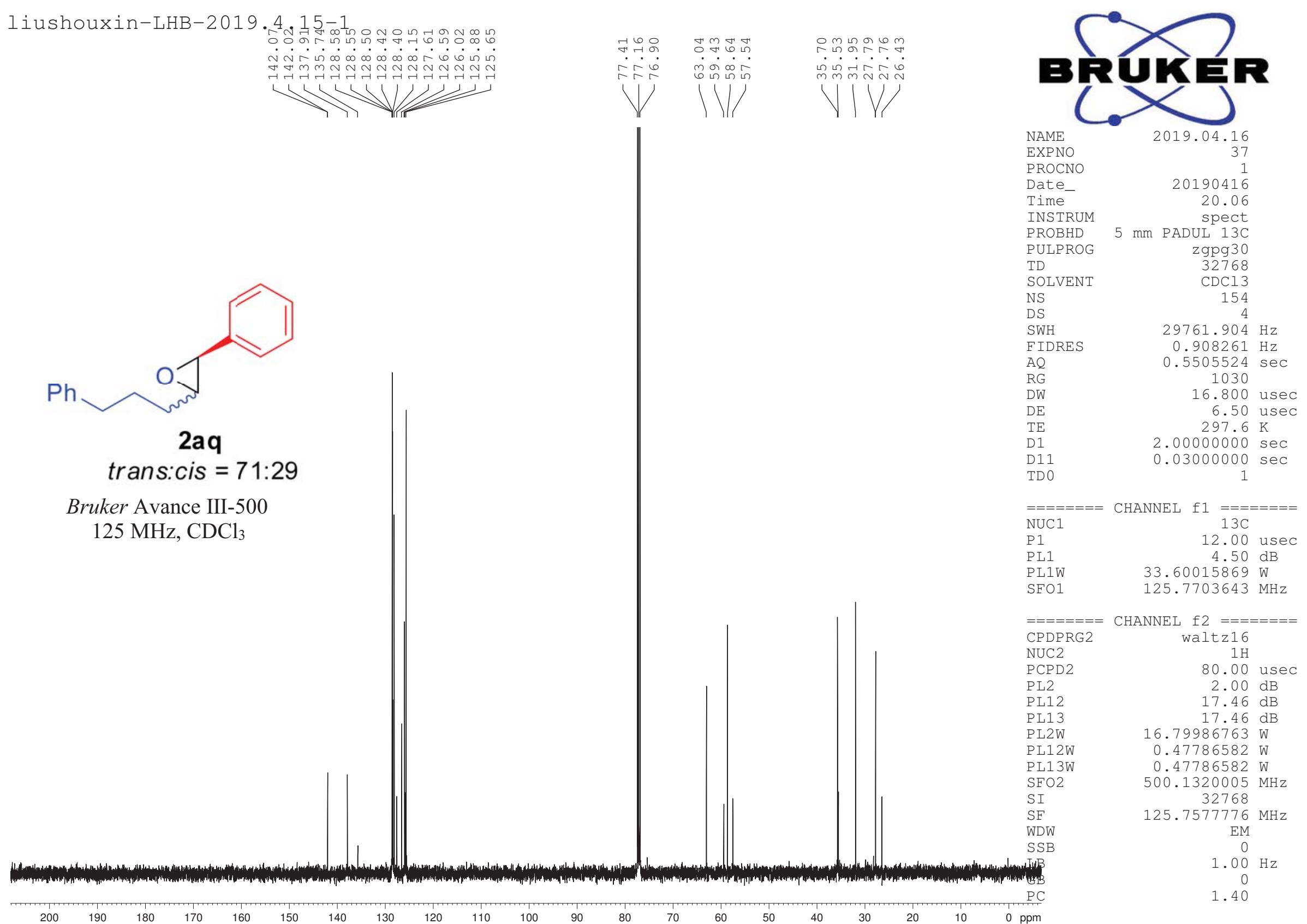



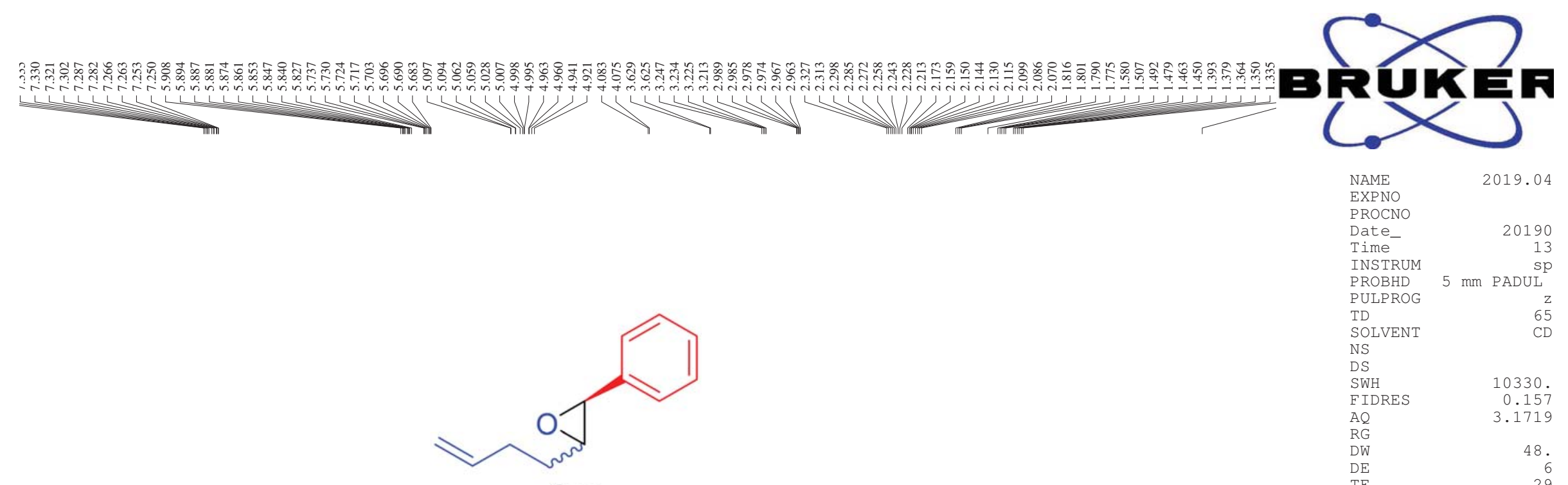

2ar

trans:cis $=72: 28$

Bruker Avance III-500

$500 \mathrm{MHz}, \mathrm{CDCl}_{3}$

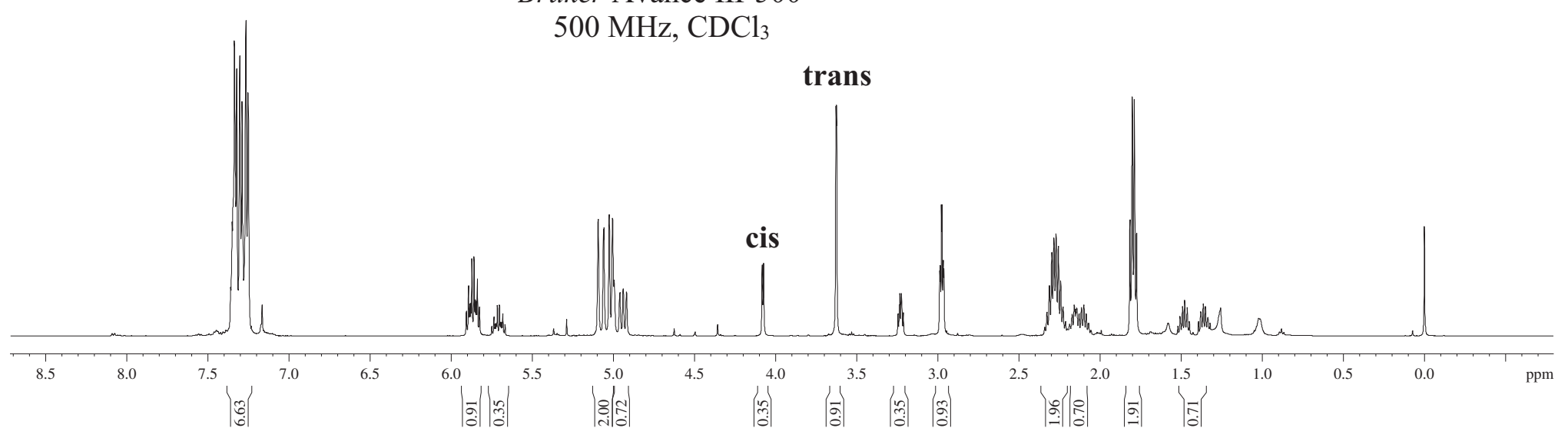

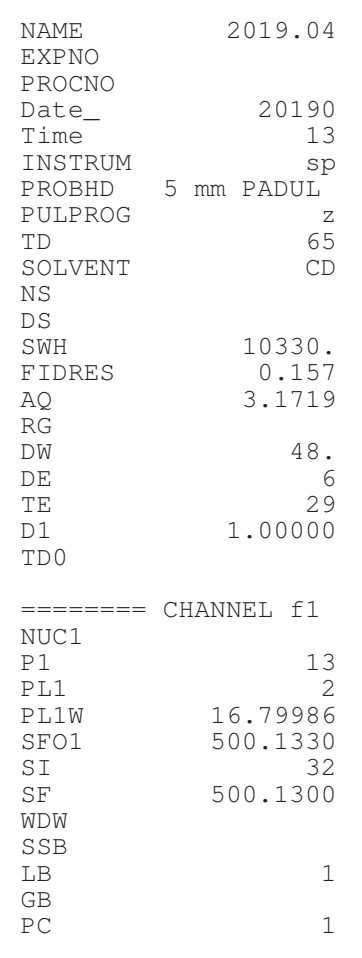




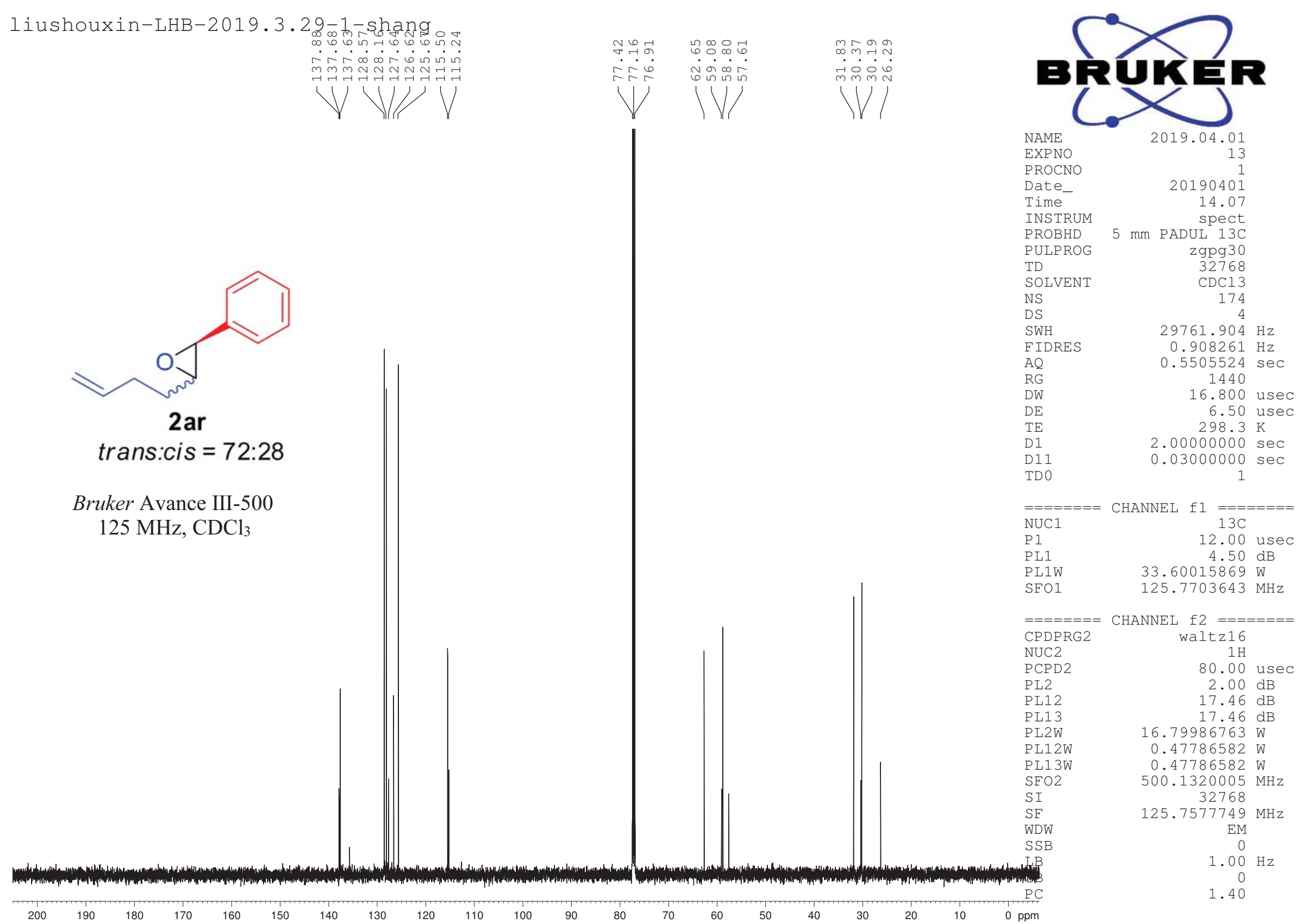




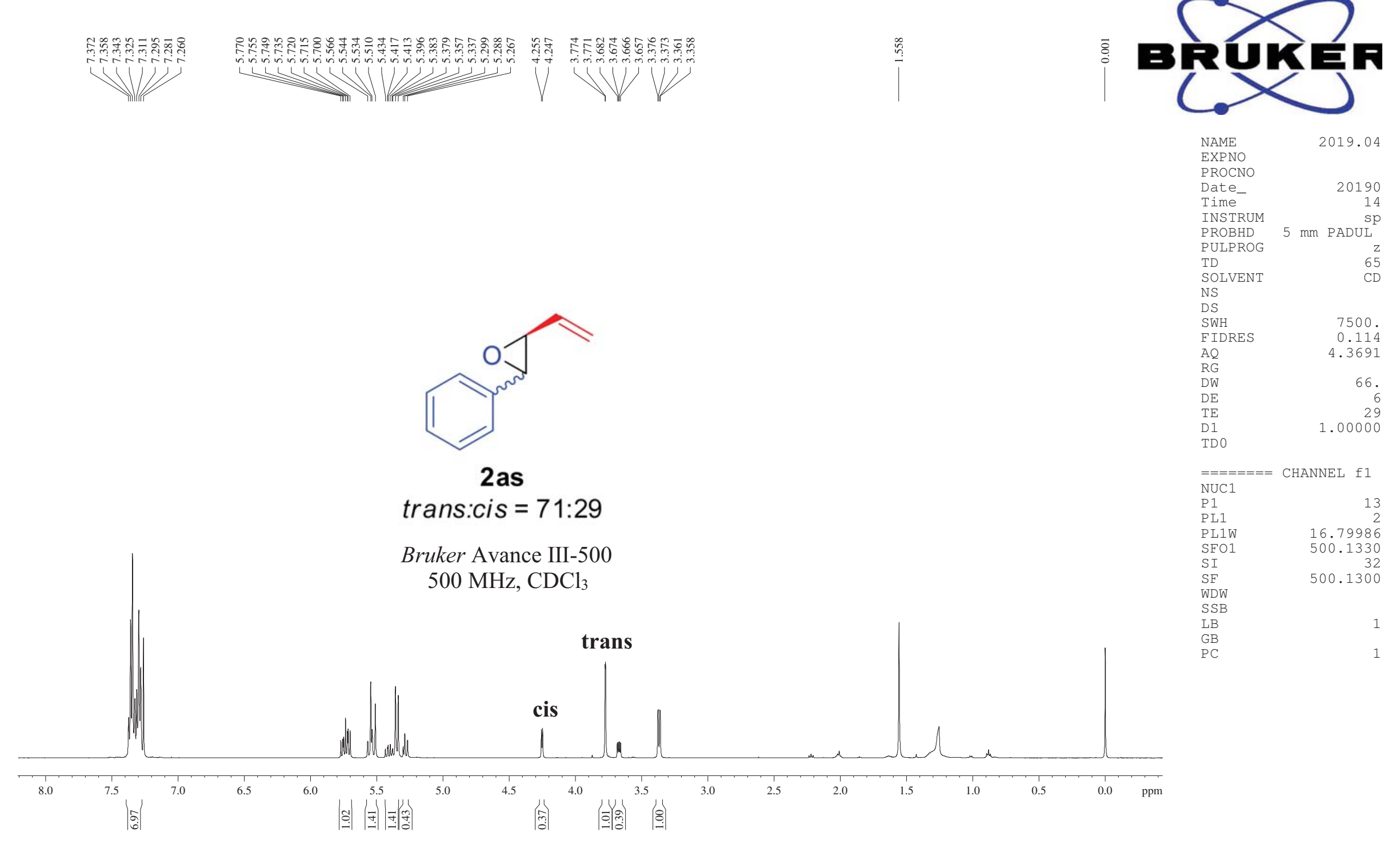




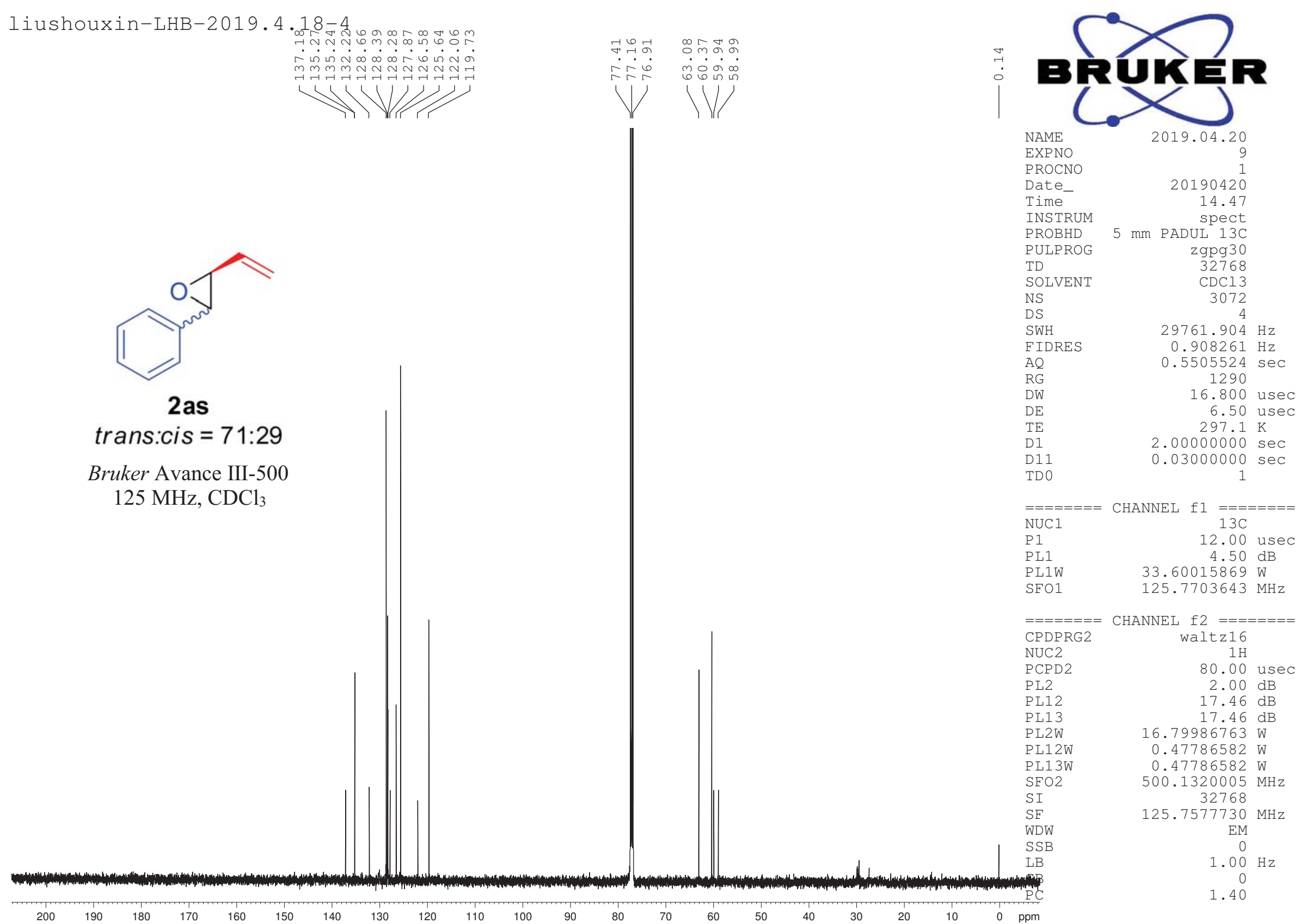




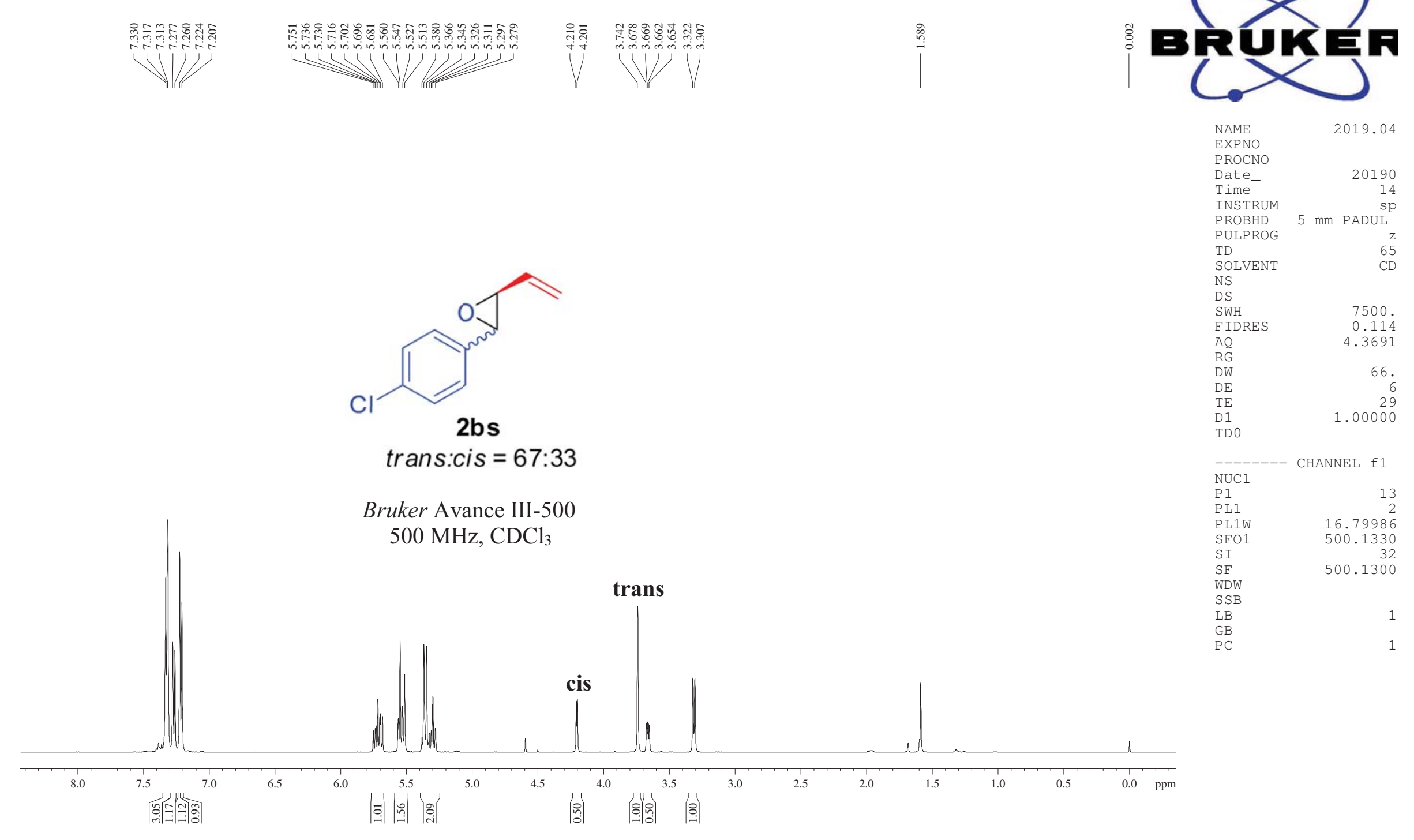




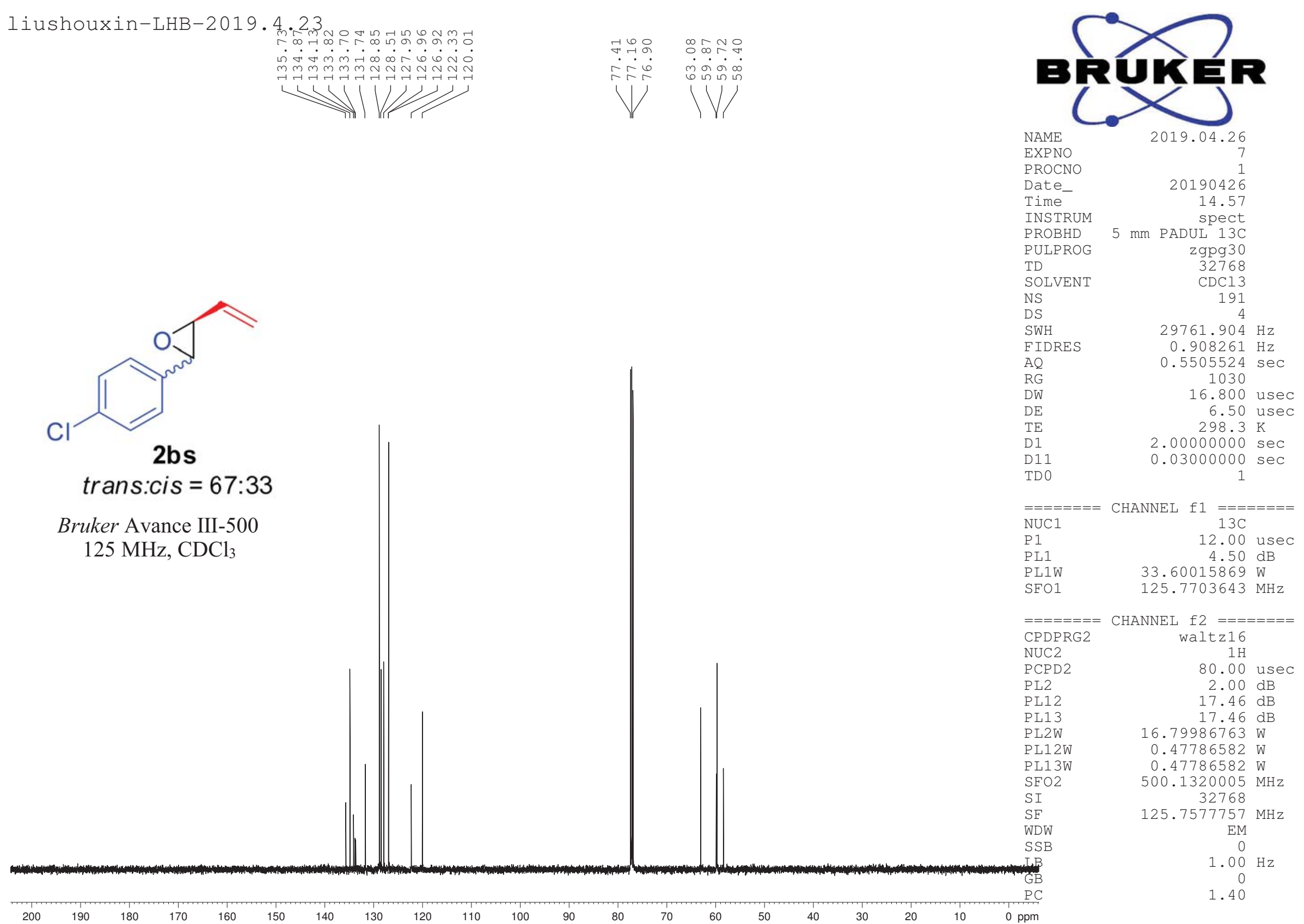




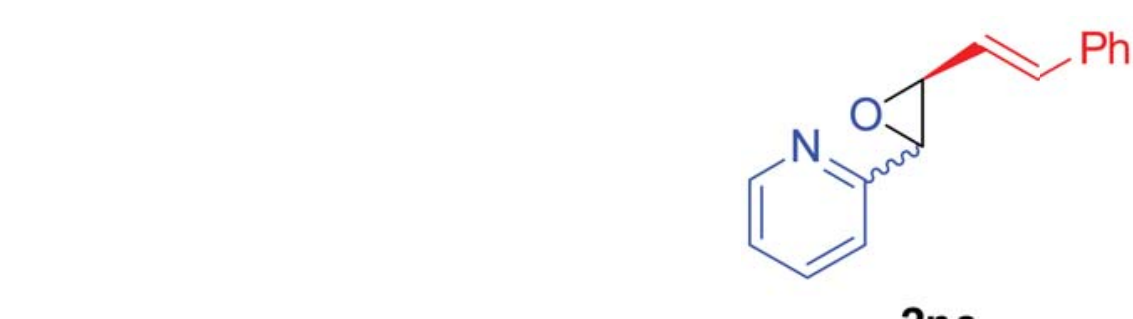

\section{2no}

trans: cis $=64: 36$

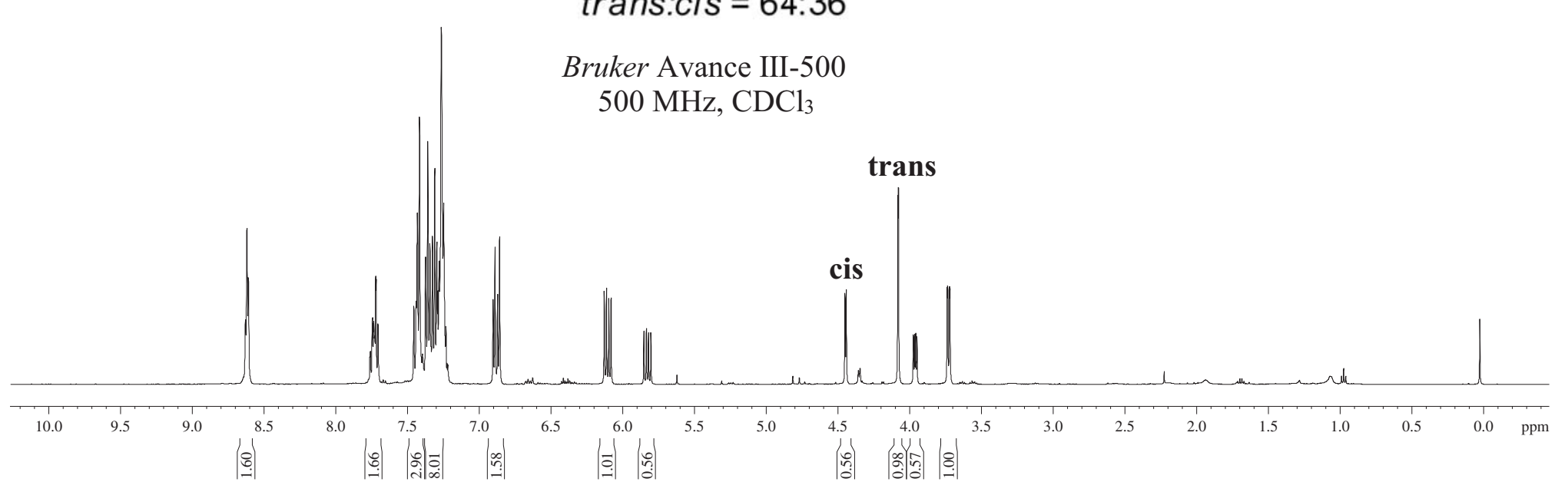

$$
\begin{aligned}
& \begin{array}{l}
\text { NAME } \\
\text { EXPNO } \\
\text { PROCNO }
\end{array} \\
& \text { Date } \\
& \text { Time } \\
& \text { PROBHD } \\
& \text { PULPROG }
\end{aligned}
$$




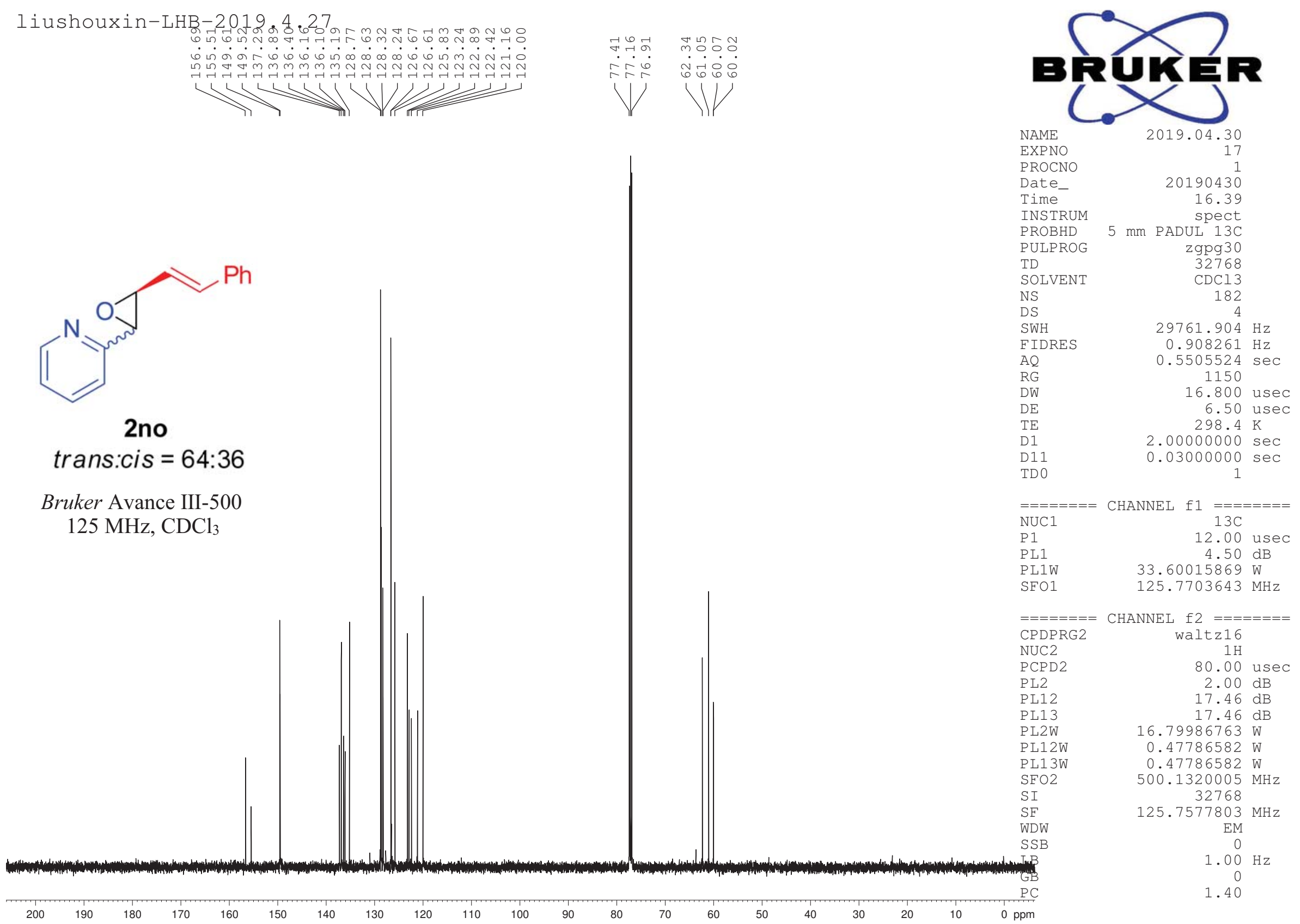

